\title{
Cochrane
}

Library

Cochrane Database of Systematic Reviews

\section{E-Health interventions for anxiety and depression in children and adolescents with long-term physical conditions (Review)}

Thabrew H, Stasiak K, Hetrick SE, Wong S, Huss JH, Merry SN

Thabrew H, Stasiak K, Hetrick SE, Wong S, Huss JH, Merry SN.

E-Health interventions for anxiety and depression in children and adolescents with long-term physical conditions.

Cochrane Database of Systematic Reviews 2018, Issue 8. Art. No.: CD012489.

DOI: 10.1002/14651858.CD012489.pub2.

www.cochranelibrary.com

E-Health interventions for anxiety and depression in children and adolescents with long-term physical conditions (Review)

Copyright @ 2018 The Cochrane Collaboration. Published by John Wiley \& Sons, Ltd. 
TABLE OF CONTENTS

HEADER

ABSTRACT

PLAIN LANGUAGE SUMMARY

SUMMARY OF FINDINGS

BACKGROUND

OBJECTIVES

METHODS

Figure 1.

RESULTS

Figure 2.

Figure 3.

DISCUSSION

AUTHORS' CONCLUSIONS

ACKNOWLEDGEMENTS

REFERENCES

CHARACTERISTICS OF STUDIES

DATA AND ANALYSES

Analysis 1.1. Comparison $1 \mathrm{E}$-health interventions vs any comparator, Outcome 1 Depression postintervention. .....................

Analysis 1.2. Comparison $1 \mathrm{E}$-health interventions vs any comparator, Outcome 2 Depression 3- to 6-month follow-up. ...........

Analysis 1.3. Comparison $1 \mathrm{E}$-health interventions vs any comparator, Outcome 3 Anxiety postintervention. ..........................

Analysis 1.4. Comparison $1 \mathrm{E}$-health interventions vs any comparator, Outcome 4 Anxiety 3- to 6-month follow-up. ................

Analysis 1.5. Comparison $1 \mathrm{E}$-health interventions vs any comparator, Outcome 5 Treatment acceptability postintervention. ..

Analysis 1.6. Comparison $1 \mathrm{E}$-health interventions vs any comparator, Outcome 6 Quality of life postintervention. .................

Analysis 1.7. Comparison $1 \mathrm{E}$-health interventions vs any comparator, Outcome 7 Quality of life 3- to 6-month follow-up. .......

Analysis 1.8. Comparison $1 \mathrm{E}$-health interventions vs any comparator, Outcome 8 Functioning postintervention. ...................

Analysis 1.9. Comparison $1 \mathrm{E}$-health interventions vs any comparator, Outcome 9 Functioning 3- to 6-month follow-up. .........

Analysis 1.10. Comparison $1 \mathrm{E}$-health interventions vs any comparator, Outcome 10 Status of long-term physical condition postintervention.

Analysis 1.11. Comparison $1 \mathrm{E}$-health interventions vs any comparator, Outcome 11 Status of long-term physical condition 3to 6-month follow-up.

Analysis 2.1. Comparison $2 \mathrm{E}$-health interventions vs attention placebo, Outcome 1 Depression postintervention. ................. Analysis 2.2. Comparison $2 \mathrm{E}$-health interventions vs attention placebo, Outcome 2 Depression 3- to 6-month follow-up. ...... Analysis 2.3. Comparison $2 \mathrm{E}$-health interventions vs attention placebo, Outcome 3 Anxiety postintervention. ....................... Analysis 2.4. Comparison $2 \mathrm{E}$-health interventions vs attention placebo, Outcome 4 Anxiety 3- to 6-month follow-up. ............. Analysis 2.5. Comparison $2 \mathrm{E}$-health interventions vs attention placebo, Outcome 5 Treatment acceptability postintervention.

Analysis 2.6. Comparison $2 \mathrm{E}$-health interventions vs attention placebo, Outcome 6 Quality of life postintervention.

Analysis 2.7. Comparison $2 \mathrm{E}$-health interventions vs attention placebo, Outcome 7 Quality of life 6-month follow-up. ............ Analysis 2.8. Comparison $2 \mathrm{E}$-health interventions vs attention placebo, Outcome 8 Functioning postintervention. ................. Analysis 2.9. Comparison $2 \mathrm{E}$-health interventions vs attention placebo, Outcome 9 Functioning 3- to 6-month follow-up. ..... Analysis 2.10. Comparison $2 \mathrm{E}$-health interventions vs attention placebo, Outcome 10 Status of long-term physical condition postintervention.

Analysis 2.11. Comparison 2 E-health interventions vs attention placebo, Outcome 11 Status of long-term physical condition 3- to 6-month follow-up.

Analysis 3.1. Comparison $3 \mathrm{E}$-health interventions vs treatment as usual (TAU), Outcome 1 Depression postintervention. ....... Analysis 3.2. Comparison 3 E-health interventions vs treatment as usual (TAU), Outcome 2 Depression 3-month follow-up. .... Analysis 3.3. Comparison 3 E-health interventions vs treatment as usual (TAU), Outcome 3 Anxiety postintervention. ............. Analysis 3.4. Comparison 3 E-health interventions vs treatment as usual (TAU), Outcome 4 Anxiety 3-month follow-up. ......... Analysis 3.5. Comparison $3 \mathrm{E}$-health interventions vs treatment as usual (TAU), Outcome 5 Functioning postintervention. ..... Analysis 3.6. Comparison $3 \mathrm{E}$-health interventions vs treatment as usual (TAU), Outcome 6 Functioning 3-month follow-up. ... Analysis 3.7. Comparison 3 E-health interventions vs treatment as usual (TAU), Outcome 7 Status of long-term physical condition postintervention. 
Analysis 3.8. Comparison 3 E-health interventions vs treatment as usual (TAU), Outcome 8 Status of long-term physical condition at 3- to 6-month follow-up.

Analysis 4.1. Comparison 4 E-health interventions vs waiting list, Outcome 1 Depression postintervention.

Analysis 4.2. Comparison $4 \mathrm{E}$-health interventions vs waiting list, Outcome 2 Functioning postintervention.

Analysis 4.3. Comparison 4 E-health interventions vs waiting list, Outcome 3 Status of long-term physical condition postintervention.

Analysis 5.1. Comparison 5 E-health interventions vs any comparison (by type of therapy), Outcome 1 Depression postintervention.

Analysis 6.1. Comparison 6 E-health interventions vs any comparator (by type of comparator), Outcome 1 Depression postintervention.

Analysis 6.2. Comparison 6 E-health interventions vs any comparator (by type of comparator), Outcome 2 Depression 3- to 6month follow-up.

Analysis 6.3. Comparison 6 E-health interventions vs any comparator (by type of comparator), Outcome 3 Anxiety postintervention.

Analysis 6.4. Comparison 6 E-health interventions vs any comparator (by type of comparator), Outcome 4 Anxiety 3-to 6-month follow-up.

Analysis 6.5. Comparison 6 E-health interventions vs any comparator (by type of comparator), Outcome 5 Treatment acceptability postintervention.

Analysis 7.1. Comparison $7 \mathrm{E}$-health interventions vs any comparator (by type of long-term physical condition (LTPC)), Outcome 1 Depression postintervention.

Analysis 8.1. Comparison $8 \mathrm{E}$-health interventions vs any comparator (by audience: child plus parent vs child only), Outcome 1 Depression postintervention.

APPENDICES

WHAT'S NEW

CONTRIBUTIONS OF AUTHORS

DECLARATIONS OF INTEREST

SOURCES OF SUPPORT

DIFFERENCES BETWEEN PROTOCOL AND REVIEW

INDEX TERMS 
[Intervention Review]

\section{E-Health interventions for anxiety and depression in children and adolescents with long-term physical conditions}

Hiran Thabrew ${ }^{1}$, Karolina Stasiak ${ }^{1}$, Sarah E Hetrick ${ }^{1,2}$, Stephen Wong ${ }^{1}$, Jessica H Huss ${ }^{3}$, Sally N Merry1

1Department of Psychological Medicine, University of Auckland, Auckland, New Zealand. 2The Centre of Youth Mental Health, University of Melbourne, Melbourne, Australia. ${ }^{3}$ Department of Psychology, University of Kassel, Kassel, Germany

Contact address: Hiran Thabrew, Department of Psychological Medicine, University of Auckland, Level 12 Support Building, Auckland Hospital, Park Road, Grafton, Auckland, New Zealand. h.thabrew@auckland.ac.nz.

Editorial group: Cochrane Common Mental Disorders Group.

Publication status and date: Edited (no change to conclusions), published in Issue 8, 2018.

Citation: Thabrew H, Stasiak K, Hetrick SE, Wong S, Huss JH, Merry SN. E-Health interventions for anxiety and depression in children and adolescents with long-term physical conditions. Cochrane Database of Systematic Reviews 2018, Issue 8. Art. No.: CD012489. DOI: 10.1002/14651858.CD012489.pub2.

Copyright @ 2018 The Cochrane Collaboration. Published by John Wiley \& Sons, Ltd.

\section{A B S T R A C T}

\section{Background}

Long-term physical conditions affect $10 \%$ to $12 \%$ of children and adolescents worldwide; these individuals are at greater risk of developing psychological problems, particularly anxiety and depression. Access to face-to-face treatment for such problems is often limited, and available interventions usually have not been tested with this population. As technology improves, e-health interventions (delivered via digital means, such as computers and smart phones and ranging from simple text-based programmes through to multimedia and interactive programmes, serious games, virtual reality and biofeedback programmes) offer a potential solution to address the psychological needs of this group of young people.

\section{Objectives}

To assess the effectiveness of e-health interventions in comparison with attention placebos, psychological placebos, treatment as usual, waiting-list controls, or non-psychological treatments for treating anxiety and depression in children and adolescents with long-term physical conditions.

\section{Search methods}

We searched the Cochrane Common Mental Disorders Group's Controlled Trials Register (CCMDTR to May 2016), the Cochrane Central Register of Controlled Trials (CENTRAL) (Issue 8, 2017), Web of Science (1900 - 18 August 2016, updated 31 August 2017) and Ovid MEDLINE, Embase, PsycINFO (cross-search 2016 to 18 Aug 2017). We hand-searched relevant conference proceedings, reference lists of included articles, and the grey literature to May 2016. We also searched international trial registries to identify unpublished or ongoing trials.

\section{Selection criteria}

We included randomised controlled trials (RCTs), cluster-randomised trials, and cross-over trials of e-health interventions for treating any type of long-term physical condition in children and adolescents (aged 0 to 18 years), and that measured changes in symptoms or diagnoses of anxiety, depression, or subthreshold depression. We defined long-term physical conditions as those that were more than three-months' duration. We assessed symptoms of anxiety and depression using patient- or clinician-administered validated rating scales based on DSM III, IV or 5 (American Psychological Association 2013), or ICD 9 or 10 criteria (World Health Organization 1992). Formal depressive and anxiety disorders were diagnosed using structured clinical interviews. Attention placebo, treatment as usual, waiting list, psychological placebo, and other non-psychological therapies were eligible comparators. 


\section{Data collection and analysis}

Two review authors independently reviewed titles, abstracts, and full-text articles; discrepancies were resolved through discussion or addressed by a third author. When available, we used odds ratio (OR) to compare dichotomous data and standardised mean differences (SMD) to analyse continuous data, both with 95\% confidence intervals (CI). We undertook meta-analysis when treatments, participants, and the underlying clinical question were adequately similar. Otherwise, we undertook a narrative analysis.

\section{Main results}

We included five trials of three interventions (Breathe Easier Online, Web-MAP, and multimodal cognitive behavioural therapy (CBT)), which included 463 participants aged 10 to 18 years. Each trial contributed to at least one meta-analysis. Trials involved children and adolescents with long-term physical conditions, such as chronic headache (migraine, tension headache, and others), chronic pain conditions (abdominal, musculoskeletal, and others), chronic respiratory illness (asthma, cystic fibrosis, and others), and symptoms of anxiety or depression. Participants were recruited from community settings and hospital clinics in high income countries.

For the primary outcome of change in depression symptoms versus any control, there was very low-quality evidence meaning that it could not be determined whether e-health interventions were clearly better than any comparator (SMD $-0.06,95 \% \mathrm{Cl}-0.35$ to 0.23 ; five RCTs, 441 participants). For the primary outcome of change in anxiety symptoms versus any comparator, there was very low-quality evidence meaning that it could not be determined whether e-health interventions were clearly better than any comparator (SMD $-0.07,95 \% \mathrm{Cl}-0.29$ to 0.14 ; two RCTs, 324 participants). For the primary outcome of treatment acceptability, there was very low-quality evidence that e-health interventions were less acceptable than any comparator (SMD 0.46, 95\% Cl 0.23 to 0.69; two RCTs, 304 participants).

For the secondary outcome of quality of life, there was very low-quality evidence meaning that it could not be determined whether ehealth interventions were clearly better than any comparator (SMD $-0.83,95 \% \mathrm{Cl}-1.53$ to -0.12 ; one RCT, 34 participants). For the secondary outcome of functioning, there was very low-quality evidence meaning that it could not be determined whether e-health interventions were clearly better than any comparator (SMD $-0.08,95 \% \mathrm{Cl}-0.33$ to 0.18 ; three RCTs, 368 participants). For the secondary outcome of status of long-term physical condition, there was very low-quality evidence meaning that it could not be determined whether e-health interventions were clearly better than any comparator (SMD $0.06,95 \% \mathrm{Cl}-0.12$ to 0.24 ; five RCTs, 463 participants).

The risk of selection bias was considered low in most trials. However, the risk of bias due to inadequate blinding of participants or outcome assessors was considered unclear or high in all trials. Only one study had a published protocol; two trials had incomplete outcome data. All trials were conducted by the intervention developers, introducing another possible bias. No adverse effects were reported by any authors.

\section{Authors' conclusions}

At present, the field of e-health interventions for the treatment of anxiety or depression in children and adolescents with long-term physical conditions is limited to five low quality trials. The very low-quality of the evidence means the effects of e-health interventions are uncertain at this time, especially in children aged under 10 years.

Although it is too early to recommend e-health interventions for this clinical population, given their growing number, and the global improvement in access to technology, there appears to be room for the development and evaluation of acceptable and effective technologically-based treatments to suit children and adolescents with long-term physical conditions.

\section{PLAIN LANGUAGE SUMMARY}

\section{E-health interventions for anxiety and depression in children and adolescents with long-term physical conditions}

\section{Why is this review important?}

More than one in ten children and adolescents worldwide have long-term physical conditions, such as asthma, diabetes, and cancer. They are more likely to develop psychological problems, which include anxiety or depression. Treating such problems early can prevent difficulties with friendships, family life, school, and future mental health problems. Accessing traditionally delivered face-to-face therapy can be difficult, due to the limited number of services. As technology improves, and therapies become available on computers and mobile telephones, e-health interventions (delivered by digital means and ranging from simple text-based programmes through to multimedia and interactive programmes, serious games, virtual reality and biofeedback programmes) may be useful to treat anxiety and depression in these children and adolescents.

\section{Who will be interested in this review?}

This review will be of interest to parents, children and adolescents, mental healthcare providers, service commissioners, and professionals caring for children with long-term physical conditions.

\section{What questions does this review aim to answer?}


This review aimed to answer the following questions: 1) Are e-health interventions better than a selected range of other therapies or waiting list in reducing symptoms of anxiety and depression in children and adolescents with long-term physical conditions? and 2) Are e-health interventions acceptable to these children and adolescents?

\section{Which studies were included in the review?}

We searched reference databases to find all randomised controlled trials, cluster-randomised trials, and cross-over trials of e-health interventions for treating anxiety or depression in children and adolescents with long-term physical conditions that were published between 1970 and August 2017. Trials had to be randomised controlled trials that included children and young people with either symptoms or formal diagnoses of anxiety or depression. We included five trials, with a total of 463 young people, in the review.

\section{What does the evidence from the review tell us?}

We included five trials of three e-health interventions (Breathe Easier Online, Web-MAP, and multimodal cognitive behavioural therapy $(\mathrm{CBT})$ ), undertaken with children aged 10 to 18 years old. Although some of these interventions were acceptable to users, none of them were clearly any better than a selected range of other therapies or waiting list at reducing symptoms of anxiety or depression.The very low quality of the evidence means the effects of e-health interventions are uncertain at this time, especially in children aged under 10 years. The review authors rated the overall risk of bias in the trials as high or uncertain.

\section{What should happen next?}

Further research should be undertaken to develop more effective e-health interventions to treat anxiety and depression in children and adolescents with long-term physical conditions. 


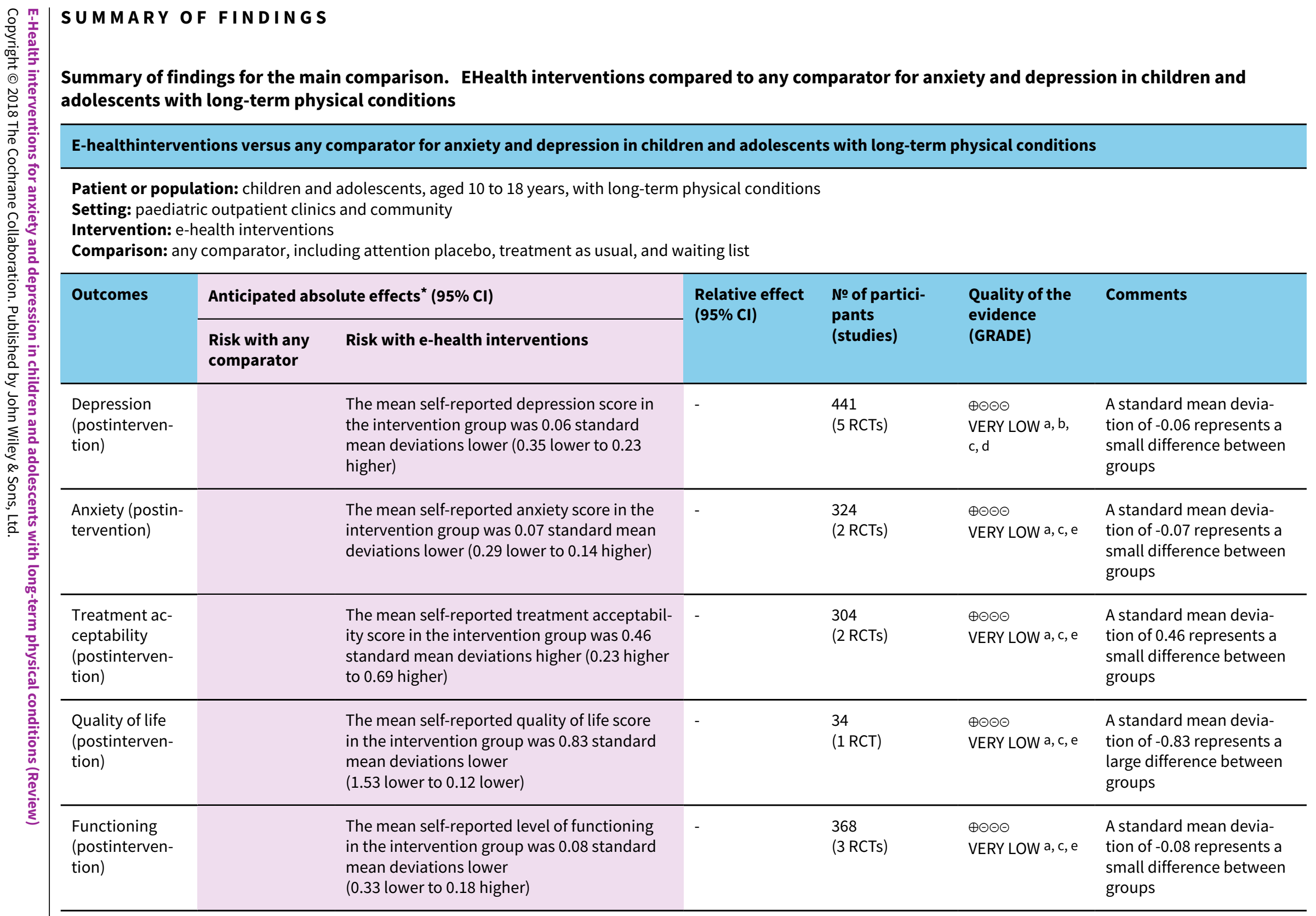


${ }^{\star}$ The risk in the intervention group (and its 95\% confidence interval) is based on the assumed risk in the comparison group and the relative effect of the intervention (and its $95 \% \mathrm{Cl})$.

Cl: Confidence interval; RR: Risk ratio; OR: Odds ratio;

\section{GRADE Working Group grades of evidence}

High quality: We are very confident that the true effect lies close to that of the estimate of the effect

Moderate quality: We are moderately confident in the effect estimate: The true effect is likely to be close to the estimate of the effect, but there is a possibility that it is substantially different

Low quality: Our confidence in the effect estimate is limited: The true effect may be substantially different from the estimate of the effect

Very low quality: We have very little confidence in the effect estimate: The true effect is likely to be substantially different from the estimate of effect

a We downgraded quality due to a lack of clarity about blinding of participants and outcome assessors, incomplete outcome data, and the fact that all studies were conducted by the developers of the e-health interventions.

$b$ We downgraded for inconsistency due to studies having moderate heterogeneity.

c We downgraded for indirectness because most or all of the interventions were not designed to treat anxiety or depression as the primary focus.

$d$ We downgraded for imprecision as the upper and lower limits of the confidence intervals include both potential for harm and potential for benefit

e We downgraded for imprecision as the total sample size was less than 400 as per guidance from the Consumer and Communication Cochrane Review Group (Ryan 2016) 


\section{B A C K G R O U N D}

\section{Description of the condition}

Long-term conditions or chronic illnesses of childhood are variably defined in the literature, but usually include physical, psychological, or cognitive problems lasting more than three months, which impair functioning (Van der Lee 2007). It is estimated that internationally, $10 \%$ to $12 \%$ of children are affected by longterm physical conditions (Eiser 1997). Asthma is the most common long-term physical condition of childhood, followed by diabetes and epilepsy (Burkart 2002). Less common long-term physical conditions include respiratory conditions, such as cystic fibrosis and bronchiectasis; cardiovascular conditions, such as congenital heart disease; gastrointestinal conditions, such as Crohn's disease; renal conditions, such as chronic kidney disease; neurological conditions, such as muscular dystrophy; chronic pain; cancer; and others (Burkart 2002). In some developed countries, the prevalence of long-term conditions is now greater than acute illnesses (Halfon 2010). Epidemiological trials show that the risk of psychological difficulties, particularly anxiety and depression, is substantially increased in children and adolescents with long-term physical conditions (Cadman 1987; Gortmaker 1990; Newacheck 1991; Opolski 2005; Pless 1971; Wallander 1995; Weiland 1992).

Anxiety disorders are common, occurring in $2.6 \%$ to $5.2 \%$ of children under 12 years, and in 5\% to $19 \%$ of all children and adolescents (Costello 2004). The presentation of anxiety disorders varies with age, from separation anxiety, undifferentiated worries, and somatic complaints in younger children, to specific phobias, panic disorder, and social anxiety in older children and adolescents. Childhood anxiety disorders often persist into adolescence and early adulthood, and yet they often remain untreated or are diagnosed late (Last 1996; Last 1997; Schneier 1992). Anxiety disorders are associated with poor academic performance, and personal and social dysfunction (Pine 2009). They may also be comorbid with depression (Kovacs 1989), substance abuse (Kushner 1990), attention deficit hyperactivity disorder (ADHD), and conduct disorder (Bittner 2007), and are associated with suicidal behaviours and death by suicide (Hill 2011). Anxiety has been identified in children and young people with long-term physical conditions as an area of clinical significance, although precise data on its incidence in this population is not available (Benton 2007; Pao 2011). It may arise from a number of different mechanisms, including confrontation by dangerous stimuli, such as threatening symptoms of illness, distressing procedures, or unpredictable events; increased fear of death in life-threatening diseases; having a reduced sense of control over one's circumstances; experiencing peer rejection or parental overprotection; and experiencing illnessspecific symptoms, such as shortness of breath in asthma (Lewis 2003; Pinquart 2011). Risk factors for developing anxiety in people with long-term conditions include younger age, female gender, and type of illness (Hermanns 2005).

Depression is another common, yet under-recognised, problem, with an overall prevalence of $0.4 \%$ to $2.5 \%$ in primary school children, and $0.4 \%$ to $8.3 \%$ in adolescents (Birmaher 1996a). A 30-year trial of American children indicated a depression rate of $2.8 \%$ in children under the age of 13 years, and of $5.6 \%$ in young people aged 13 to 18 years (Costello 2004). Rates rise rapidly during adolescence (Feehan 1993; Feehan 1994; Fergusson 1993; Fergusson 2001). By the age of 19 years, between a fifth and a quarter of young people have suffered from a depressive disorder (Lewinsohn 1998; Rhode 2013). Depression is associated with poor academic performance, social dysfunction, substance abuse, and attempted and completed suicide (Birmaher 1996a; Birmaher 1996b; Brent 1986; Brent 2002; Fleming 1993; Rao 1995; Rhode 1994). Even subthreshold depression is associated with an increased risk of later depression (Gonzales-Tejera 2005), substance abuse (Judd 2002), suicidal behaviours (Fergusson 2006), and mortality (Cuijpers 2002). Depression may be comorbid with anxiety in $15.9 \%$ to $61.9 \%$ of children identified as either anxious or depressed, and measures of anxiety and depression are highly correlated (Brady 1992). Depression has also been identified as occurring more commonly in children and adolescents with longterm physical conditions, although precise data on its incidence in this population is not available (Dantzer 2003; Pinquart 2011). Depressive symptoms have been reported in as many as $40 \%$ of children with a long-term condition and socialisation problems (Denny 2014). Risk factors for depression in long-term conditions are thought to include low self-esteem and a negative attributional style (Burke 1999).

\section{Description of the intervention}

Psychological interventions are defined as any psychotherapeutic treatment (talking therapy) specifically designed to change cognition, behaviour, or both, with the intention of improving mental health outcomes (Eccleston 2012). Evidence regarding interventions for psychological problems in children with longterm physical conditions is limited (Compas 2012). The majority of interventions specifically designed for children and adolescents with long-term physical conditions focus on compliance with medical treatment, education about the medical condition, and improving aspects of medical care (Fielding 1999; Smith 1986). Psychological issues, especially anxiety and depression, are usually addressed using standard psychological treatments, which may or may not have been tested in this population. Access to such therapies may be limited, depending upon the availability of community child and adolescent mental health services, paediatric consultation liaison services, and other community-based health services.

E-health is an emerging and fast-developing field of research and practice that involves the application of digital technologies (i.e. those delivered via digital means, such as computers and smart phones) to support or deliver health interventions. Ehealth programmes have many advantages: the fidelity of the intervention process is embedded in the programme, patients can access treatment at their convenience, and they can work at their own pace, in privacy. Computers may be preferable for some who are unable (e.g. those living in rural areas) or reluctant (e.g. many adolescents) to seek traditional face-to-face care (Fleming 2015). E-health interventions can take various forms, from reasonably simple, predominantly text-based programmes (e.g. websites offering information), through multimedia and interactive programmes that can incorporate emails or text messages, all the way to sophisticated applications, such as virtual reality systems (e.g. used as a distraction to reduce pain in children; Law 2011). They may also include serious games (Fleming 2015), and biofeedback programmes that use galvanic skin response and heart variability sensors, to detect stress-related physiological changes, e.g. used for stress management (Pop-Jordanova 2010), or relaxation training (Amon 2008). 
Given the greater likelihood of psychological issues in children and adolescents with long-term physical conditions, and the increasing availability of e-health technology, it is pertinent to consider the value of e-health-based psychological therapies and interventions in addressing these conditions, whether the computer programmes are of generic design or specifically designed for this population. A growing body of evidence suggests that computer-delivered interventions are feasible and potentially efficacious in delivering compliance- and treatmentrelated behavioural therapies to children and adolescents with long-term physical conditions, and their families (Stinson 2009). Furthermore, a review of 15 trials has suggested that children with chronic health conditions may be less likely to drop out from computerised interventions than from face-to-face interventions (Dunn 2011). The UK's National Institute for Health and Care Excellence (NICE) endorsed computerised interventions (based on cognitive behavioural therapy (CBT)) as the preferred first line of treatment for mild to moderate depression and anxiety (NICE 2006). There is limited evidence that computerised CBT may be useful for treating depression in adults with long-term physical conditions (Sharp 2014). Whether or not this is the same for children and adolescents with long-term physical conditions remains to be determined, as does the effectiveness of other models of computerised psychotherapy with this population.

\section{How the intervention might work}

The aetiologies of both anxiety and depression are complex, and include biological, psychological, and social factors (Cicchetti 1998; Davidson 2002; Goodyer 2000; Lewinsohn 1994; McCauley 2001). Although modalities, such as behaviour therapies (Martell 2001), third wave CBTs (Hayes 2004), psychodynamic therapies (McQueen 2008), humanistic therapies, integrative therapies (Mufson 2004), and systemic therapies (Carr 2006), may all be used to treat these conditions in face-to-face settings, we anticipate that the majority of e-health interventions designed to address anxiety and depression are likely to be based upon the principles of CBT, and to include an element of education about the psychological problem being addressed. Potential mechanisms for the main categories of psychological therapies are as follows.

Behaviour therapies aim to constructively change patients' behaviour towards their symptoms using operant conditioning. Common components used to treat anxiety and depression include psycho-education (Guerney 1971), relaxation training (Lowe 2002), and behavioural activation (BA (Jacobsen 1996; Martell 2001)). Biofeedback techniques may also be used (Schwartz 2003).

CBT helps to link thoughts, feelings, and behaviour, and target the situations or triggers that generate emotional responses. Cognitive appraisal of triggers and altering cognitions, in order to change mood and behaviour, are supported. CBT for depression is based on the cognitive model of depression that proposed that individuals prone to depression have cognitive distortions, which result in a negative view of themselves, the world, and the future (Beck 1976). People with pessimistic 'attribution styles' have a bias toward viewing negative events as stable and self-induced, versus positive events as transient and out of their control (Abramson 1978). This leads to a state of 'learned helplessness' and hopelessness (Petersen 1993; Seligman 1979), as well as passivity, in the face of challenges (McCauley 2001). CBT for depression in children and adolescents involves helping the child to: (1) recognise and evaluate their thoughts, and identify different levels of mood in themselves, (2) recognise thoughts and behaviours that have contributed to this mood, (3) develop coping strategies to address them via effective problem-solving, and (4) evaluate outcomes. CBT has been shown to improve depression in children and adolescents (Harrington 1998; Reinecke 1998; Weisz 2017), and prevent relapse (Paykel 1999), although long-term results in trials have contradictory findings (Fonagy 2005). CBT for anxiety is based on Beck's cognitive model of anxiety, which proposes that fear and anxiety are learned responses that can be 'unlearned'. CBT for anxiety in children and adolescents involves helping the child to: (1) recognise anxious feelings and bodily reactions, (2) clarify thoughts or cognitions in anxiety-provoking situations, (3) develop effective coping skills via modified self-talk, modelling, reality or in vivo exposure, role playing, and relaxation training, and (4) evaluate outcomes (Silverman 1996). An element of treatment, known as systematic desensitisation, involves pairing anxiety stimuli, in vivo or by imagination, in a gradually-increasing hierarchy with competing relaxing stimuli, such as pleasant images and muscle relaxation (James 2013). Recent advances have identified optimal methods of delivering exposure work, including deepened extinction, variability, and affect labelling (Craske 2014).

Third wave CBTs include acceptance and commitment therapy (ACT (Hayes 1999; Hayes 2004)), compassionate mind training (CMT), also known as compassion-focused therapy (Gilbert 2005; Gilbert 2009), functional analytic psychotherapy (FAP (Kohlenberg 1991)), metacognitive therapy for depression (Wells 2008; Wells 2009), and dialectical behaviour therapy (Koons 2001; Linehan 1993). These approaches use a combination of cognitive, behavioural, and mindfulness techniques to assist people to manage situations without thought suppression or experiential avoidance (Hoffman 2008).

Psychodynamic therapies aim to resolve internal conflicts stemming from difficulties in past relationships and experiences (for example, sexual abuse). Such conflicts are thought to cause anxiety or psychic pain, and are 'repressed' into the unconscious through the use of defence mechanisms (Bateman 2000). Although some defence mechanisms are adaptive, some are developmentally immature, and can cause harm. Psychoanalytic (sometimes called psychodynamic) psychotherapy attempts to explore, through talking, playing (with younger children), and forming a therapeutic relationship, how earlier experiences influence and perhaps seriously distort current thoughts, feelings, behaviours (actions), and relationships (McQueen 2008).

Humanistic therapies include grief therapy, supportive therapy, and transactional analysis. These therapies are based on the premise that people are 'self-actualising', that is, they have an inherent tendency to develop their potential, and they are selfaware, free to choose how they live, and are responsible for the choices they make (Rogers 1951; Maslow 1970). Individualised, rather than manualised or prescribed methods, are undertaken to help them address their situation (Cain 2002).

Integrative therapies include interpersonal therapy (IPT), which addresses interpersonal conflict, difficulty with role transitions, and experiences of loss, all of which are well-known risk factors for the development of depressive disorders in young people (Birmaher 1996a; Lewinsohn 1994; McCauley 2001). Preponents have proposed that IPT works by activating several interpersonal change mechanisms, including: (1) enhancing social support, (2) decreasing interpersonal stress, (3) facilitating emotional 
processing, and (4) improving interpersonal skills (Lipsitz 2013). It has been shown to be effective in the treatment of teenage depression (Bolton 2007; Mufson 1996; Mufson 2004).

Systemic therapies include family therapy, which is based on the premise that family members can influence one another's wellbeing, and have a significant effect on both the development of symptoms, and the outcomes of interventions (Carr 2006). There are a number of forms of family therapy, including structural family therapy, which centres on individual physiological vulnerability, dysfunctional transactional styles, and the role the sick child plays in facilitating conflict avoidance (Liebman 1974; Minuchin 1978). Systems therapy, including Milan and post-Milan family therapy, attempts to elicit changes in the family dynamic, by presenting information that encourages family members to reflect on their own behaviour within the family dynamic (Selvini 1978). Strategic family therapy acknowledges the effect of the illness on all family members, and focuses on inducing a change in symptoms by highlighting paradoxical intentions of family members (Madanes 1981). Attachment-based family therapy (ABFT) combines elements of attachment theory and family systems theory, and parents are encouraged to sensitively respond to young people. It has been shown to be better than waiting-list control for treating depression, and to lead to faster resolution of depressive symptoms, and less suicidal ideation than waiting-list control (Diamond 2002). ABFT has also been shown to lead to greater client and family satisfaction and retention when combined with CBT, than when CBT is used alone for treating anxiety in young people (Siqueland 2005).

Delivery of these psychological interventions via digital makes them potentially more cost-effective and widely available. They are able to be accessed by those who may otherwise not engage in treatment, and mean that people can work at their own pace, access treatment as and when they need it, and do so in privacy.

\section{Why it is important to do this review}

As the field of e-health is a relatively new one, the evidence base regarding the effectiveness of e-health interventions, especially in a population, such as people with long-term conditions, is currently limited. This review aims to fill a gap in the literature by identifying and evaluating randomised controlled trials (RCTs) of e-healthbased interventions that directly or indirectly address anxiety or depression in children and adolescents with long-term physical conditions. Establishing this evidence base will inform the clinical use of existing effective resources, and guide the development of newer and potentially more cost-effective and globally dispersible forms of treatment for this growing population.

Due to the unique qualities of e-health interventions, and the rapidly growing nature of this new field of healthcare, e-health interventions for addressing anxiety and depression in children and adolescents with long-term physical conditions are being considered separately from non-e-health interventions by the same authors in a related review (Thabrew 2017a). This review also sits alongside a review of serious games for treating depression in children and adolescents who do not have a long-term condition (Fleming 2015). A few existing Cochrane reviews have already investigated the value of psychological therapies for anxiety and depression in adults, children, and adolescents (Barak 2008). Of the latter, one review addressed the prevention of depression in children and adolescents without specifically addressing those with long-term conditions (Hetrick 2016). Two reviews addressed the treatment of depression (Cox 2014), and anxiety (James 2013), in children and adolescents, but again not specifically in those with long-term conditions. Two reviews have addressed psychological interventions for depression in adolescents who have a single condition, such as congenital heart disease (Lane 2013), or pain (Eccleston 2014), and one review has focused on interventions for parents, rather than for children (Eccleston 2012).

\section{OB JECTIVES}

To assess the effectiveness of e-health interventions in comparison with attention placebos, psychological placebos, treatment as usual, waiting-list controls, or non-psychological treatments for treating anxiety and depression in children and adolescents with long-term physical conditions.

\section{METHODS}

\section{Criteria for considering studies for this review Types of studies}

We included all randomised controlled trials (RCTs) and clusterrandomised trials. We also included cross-over trials, only using the data from the first phase, in order to avoid carry-over effects. We excluded observational trials, quasi-randomised trials, and nonrandomised trials. We did not exclude any trial on the basis of language of publication or publication status.

\section{Types of participants}

\section{Age}

We included trials involving children and adolescents aged 0 to 18 years (or those that had at least $80 \%$ of the sample within this age range).

\section{Diagnosis}

We included trials whose participants had any single or mixed long-term physical condition of more than three-months' duration, and measurable symptoms of anxiety, depression, or subthreshold depression. Symptoms of anxiety and depression were assessed using patient or clinician-administered validated rating scales (Sadock 2005), based on DSM III, IV or 5 (American Psychological Association 2013), or ICD 9 or 10 (World Health Organization 1992) criteria. Formal depressive and anxiety disorders were diagnosed using structured clinical interviews.

\section{Comorbidities}

We included trials with participants with any mixed, longterm conditions, and with both anxiety and depression. We included trials of participants who may also have had any type of comorbid physical condition (e.g. asthma, diabetes, epilepsy), or another mental health condition (e.g. attention deficit hyperactivity disorder, obsessive compulsive disorder, schizophrenia).

\section{Setting}

We included trials involving those treated in hospital or community settings. 


\section{Types of interventions}

\section{Experimental intervention}

Experimental interventions included any e-health intervention that had measured changes in anxiety or depression, and that had been tested in children and adolescents with long-term conditions. These may have been delivered via the Internet (e.g. static or interactive websites, automated emails, or web-based applications), mobile telephones (e.g. automated phone calls or short text messages), or smartphones (e.g. mobile websites or smartphone applications). These may have been entirely individually used (self-help) or therapist-supported, and may have included parental participation, but not telemental health, where psychological intervention was provided remotely, via telephone, chatroom, email, or videoconferencing, and not interventions that were designed only for parents. Eligible modalities of therapy included the following.

1. Cognitive behavioural therapy (CBT (Harrington 1998; Reinecke 1998; Weisz 2006)).

2. Behaviour therapies (e.g. relaxation training (Lowe 2002)).

3. Third wave CBTs (e.g. acceptance and commitment therapy (Hayes 1999)).

4. Other psychologically-oriented therapies (e.g. mixed models of therapy, such as CBT plus relaxation training).

\section{Comparator intervention}

Comparator interventions included any of the following.

1. Attention placebo (AP): a control condition in which the control group received an intervention that mimicked the time and attention received by the intervention group, but was not thought to be active.

2. Treatment as usual (TAU): participants could receive any appropriate medical care during the course of the trial on a naturalistic basis, including standard psychological or pharmacotherapeutic care, usual care, or no treatment.

3. Waiting list (WL): as in TAU, patients in the WL- control could receive any appropriate medical care during the course of the trial on a naturalistic basis.

4. Psychological placebo (PP): a control condition that was regarded as inactive in a trial by researchers, but was regarded as active by the participants.

5. Other non-psychological therapies (e.g. pharmacotherapy for depression or anxiety).

\section{Main planned comparisons}

1. e-health interventions for anxiety or depression versus any comparator

2. e-health interventions for anxiety or depression versus attention placebo (AP)

3. e-health interventions for anxiety or depression versus treatment as usual (TAU)

4. e-health interventions for anxiety or depression versus waiting list (WL)

5. e-health interventions for anxiety or depression versus psychological placebo (PP)
6. e-health interventions for anxiety or depression versus other non-psychological therapies (e.g. pharmacotherapy for depression or anxiety)

\section{Types of outcome measures}

We focused outcome measures on the individual child rather than the wider family. We evaluated the difference between the treatment group and the control group separately for anxiety and depression, using the following outcomes.

\section{Primary outcomes}

1. Treatment efficacy: we measured changes in severity of anxiety and depression symptoms separately, using validated scales for each of these conditions (e.g. Children's Depression Inventory (CDI) for childhood depression (Kovacs 1989); State-Trait Anxiety Inventory (STAI) for anxiety (Spielberger 1983)). We analysed clinician-rated scales separately from those rated by the children, young people, parents, and others (e.g. teachers). We interpreted statistically-significant results by taking into account the clinical significance of each scale (using T-scores if these were available for all scales).

2. Treatment acceptability: as reported by quantitative measures of treatment acceptability (e.g. the Treatment Evaluation Inventory-Short Form (Newton 2004)), the number of participants who dropped out for any reason, and because of adverse events.

\section{Secondary outcomes}

1. Changes in caseness (remission or response): we measured these separately, using similar validated scales for each of the conditions.

2. Suicide-related behaviour: we assessed as the number of a) deaths by suicide, b) suicide attempts, and c) episodes of deliberate self harm, either reported or measured, using validated scales (Osman 2001).

3. Improvement in quality of life: we measured using validated scales (e.g. Paediatric Quality of Life inventory (PedsQL (Varni 2004)).

4. Functioning, as a proxy for psychological well-being: we measured using validated scales (e.g. Children's Global Assessment Scale (CGAS (Shaffer 1984)).

5. Status of long-term physical condition: we measured using validated scales (e.g. Paediatric Asthma Symptom Scale (PASS (Lara 2000)). Note: As the only physical outcome that was available was a change in pain, we labelled the outcome 'Pain' in the analysis section.

6. Adherence to treatment of long-term physical condition.

7. School or college attendance (e.g. reduction in number of days missed).

8. Economic benefits (e.g. reduction of costs of treatment, number of appointments with general practitioners, use of additional treatments, ability to study or work).

\section{Timing of outcome assessment}

We undertook clustering and comparison of outcome measures at similar time periods. The primary time point was short-term change (i.e. at the end of treatment). We assessed short-term and long-term (three months or more beyond the end of treatment) outcome measures separately. If multiple long-term measures had 
been provided, we had planned to use the one furthest from the intervention, as this was most relevant to understanding the enduring nature of the therapeutic effect.

\section{Hierarchy of outcome measures}

For trials presenting a range of symptom measures (e.g. multiple depression scales), we used the scale ranked highest according to the following five criteria: appropriateness to children and adolescents, reliability, construct validity, agreement with clinical interview, and track record in psychopharmacological research.

For depression, we ranked them, from highest to lowest, as follows: Schedule for Affective Disorders and Schizophrenia for SchoolAge Children (Kiddie-SADS (Kaufman1997)), Children's Depression Rating Scale (CDRS (Poznanski 1985)), Bellevue Index of Depression (BID (Petti 1978)), Children's Depression Inventory (CDI (Kovacs 1985)), Hamilton Depression Rating Scale (HAM-D (Hamilton 1967)), Depressive Adjective Checklist (DACL (Lubin 1965)), then others (Hazell 2002).

For anxiety, we ranked them, from highest to lowest, as follows: Anxiety Disorder Interview Schedule (ADIS (Silverman 1988)), Multidimensional Anxiety Scale for Children (MASC (March 1997)), Paediatric Anxiety Rating Scale (PARS (PARS 2002)), Social Phobia and Anxiety Inventory for Children (SPAI-C (Beidel 2000)), Social Anxiety Scale for Children-Revised (SASC-R (La Greca 1988)), Fear Survey Schedule for Children-Revised (FSSC (Olendick 1983)), Revised Children's Manifest Anxiety Scale (RCMAS (Reynolds 1978)), State-Trait Anxiety Inventory for Children (STAI-C (Spielberger 1973)), Screen for Child Anxiety-Related Emotional Disorders (SCARED (Birmaher 1999)), Hamilton Anxiety Rating Scale (HARS (Maier 1988)), then others (based on Myers 2002).

\section{Search methods for identification of studies}

\section{Cochrane Common Mental Disorders Controlled Trials Register (CCMD-CTR)}

The Cochrane Common Mental Disorders Group maintained a specialised register of randomised controlled trials, the CCMDCTR (to June 2016). This register contains over 40,000 reference records (reports of RCTs) for anxiety disorders, depression, bipolar disorder, eating disorders, self harm, and other mental disorders within the scope of the Cochrane Common Mental Disorders Group (CCMDG). The CCMD-CTR is a partially trials-based register with more than $50 \%$ of reference records tagged to approximately 12,500 individually PICO-coded trial records. Reports of trials that are included in the register were collated from (weekly) generic searches of MEDLINE (from 1950), Embase (from 1974), and PsycINFO (from 1967), quarterly searches of the Cochrane Central Register of Controlled Trials (CENTRAL), and review-specific searches of additional databases. Reports of trials were also sourced from international trial registries, drug companies, the handsearching of key journals, conference proceedings, and other (non-Cochrane) systematic reviews and meta-analyses. Details of CCMDG's core search strategies used to identify RCTs, can be found on the Group's website. We have included an example of the core MEDLINE search in Appendix 1.

The register fell out of date with the Editorial Group's move from Bristol to York in the summer of 2016
The CCMDCTR was current to 16 May 2016 when we last ran the search.

\section{Electronic searches}

Searches for this review have been through a number of iterations. The Group's Information Specialist initially ran a broad search of the Cochrane Common Mental Disorders Controled Trials Register (CCMDCTR), using the following terms (16 May 2016). The search of the CCMDCTR was not repeated in August 2017 as the register was out-of-date at this time.

\section{CCMDCTR-Studies register}

Condition $=$ (anxiety or depressi ${ }^{\star}$ or mood or mutism or neuroses or neurotic or "obsessive compulsive" or panic or "phobi" or psychoneuroses or "stress disorder*" or "psychological stress" or "school refusal")

and Comorbidity = not empty

and Age Group = (child or adolescent)

We screened these records for e-health-based interventions in this population.

\section{CCMDCTR-References register}

The Information Specialist searched the references register, using a more sensitive set of terms, to find additional untagged and uncoded reports of RCTs (Appendix 2).

The CCMD's Information Specialist conducted complementary searches on the following bibliographic databases, using relevant subject headings (controlled vocabularies) and search syntax, appropriate to each resource.

- The Cochrane Central Register of Controlled Trials (CENTRAL) via the Cochrane Register of Studies Online (CRSO; searched 9 June 2016 and 18 August 2017 (Appendix 3)).

- Other Cochrane Library databases (CDSR, DARE, HTA; searched 9 June 2016 and 18 August 2017)

In August 2017, the Information Specialist ran a search of CENTRAL (2017, Issue 8), and a cross-search of Ovid MEDLINE, Embase and PsycINFO (searched 18 August 2017 (Appendix 4)).

We searched the following resources:

- Web of Scence Core Collection (Science, Social Science and Conference Proceeding indices ( $\mathrm{SCl}, \mathrm{SSCl}, \mathrm{CPCl}-\mathrm{S}, \mathrm{CPCl}-\mathrm{SSH}$; searched 18 August 2016 and 31 August 2017) (employing the same search strategy as displayed in Appendix 2, but amending NEXT to NEAR/x and adding an RCT filter (random* OR "cross over" OR crossover or trial OR trials).

- International trial registries via the World Health Organization's trials portal (ICTRP) and ClinicalTrials.gov pm 27 May 2016 and 29 August 2018 to identify unpublished or ongoing trials.

We did not apply any restrictions on date, language or publication status to the searches. 


\section{Searching other resources}

\section{Handsearching}

We handsearched relevant conference proceedings (those titles not already indexed in Embase or PsycINFO, or already handsearched for CENTRAL) as follows:

- Annual Meeting of the American Academy of Child and Adolescent Psychiatry (AACAP; searched from 2000 onwards); and

- International Conference of the European Federation for Medical Informatics (MIE; searched via Studies in Health Technology and Informatics journal).

\section{Reference lists}

We checked the reference lists of all included trials and relevant systematic reviews to identify additional trials, missed from the original electronic searches (for example, unpublished or in-press citations).

\section{Grey literature}

We searched sources of grey literature via the following websites: Open Grey www.opengrey.eu/ and the National Guidlines Clearing House www.guideline.gov/

\section{Correspondence}

We contacted authors of included trials, and subject experts for information on unpublished or ongoing trials.

\section{Data collection and analysis}

\section{Selection of studies}

Two authors (HT and SW), in conjunction with the CCMDG editorial office, conducted the searches. Two authors (HT and JH) independently screened the titles and abstracts of the records identified. They discarded trials that obviously did not fulfil inclusion criteria at this stage of the screening process. We retrieved the full texts of eligible or potentially-eligible trials for independent full-text inspection by two authors (HT and $\mathrm{JH}$ ). We resolved any discrepancies by discussion, or by involving a third author (KS) as necessary. We listed the reasons for exclusion in the 'Characteristics of excluded trials' table. We kept notes that described the selection process in enough detail to complete a PRISMA flow diagram (Figure $1)$. 
Figure 1. Study flow diagram.

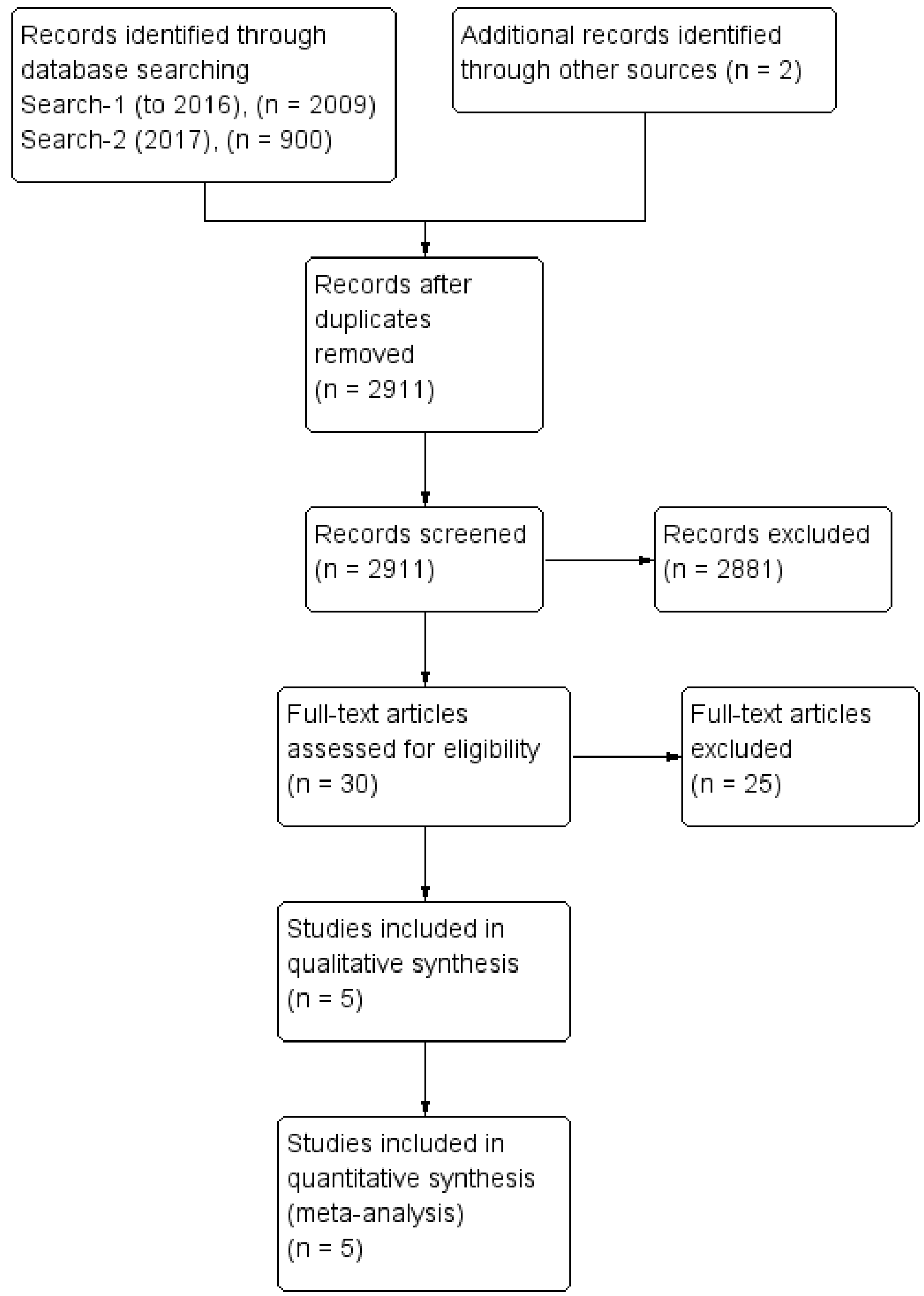

E-Health interventions for anxiety and depression in children and adolescents with long-term physical conditions (Review) 


\section{Data extraction and management}

Two authors (HT and KS) independently extracted data on trial characteristics, methodology, participant characteristics, intervention characteristics, outcome measures, and outcome data, using Covidence ${ }^{\circledR}$ software (Covidence). We contacted authors to obtain additional information when required. After agreement, one author (HT) transferred data into RevMan 5.3 for analysis (RevMan 2014). We used the format that would allow us to include the maximum numbers of trials (events and total number of patients for each group; mean, standard deviations (SDs), and number of patients included in each group; or generic inverse variance if necessary). We resolved disagreements by discussion, or with the help of the third author (SH).

\section{Assessment of risk of bias in included studies}

We assessed risk of bias for each included trial, using Cochrane's 'Risk of bias' tool (Higgins 2011). We considered the following sources of bias.

1. Sequence generation: was the allocation sequence adequately generated?

2. Allocation concealment: was allocation adequately concealed?

3. Blinding of participants and care providers for each main outcome or class of outcomes: was knowledge of the allocated treatment adequately prevented during the trial?

4. Blinding of outcome assessors for each main outcome or class of outcomes: was knowledge of the allocated treatment adequately prevented during the trial?

5. Incomplete outcome data for each main outcome or class of outcomes: did more than $10 \%$ of participants withdraw and were incomplete outcome data adequately addressed? This figure was used in a previous review of the prevention of depression in children and adolescents (Hetrick 2016).

6. Selective outcome reporting: are reports of the trial free of any suggestion of selective outcome reporting?

7. Other sources of bias: was the trial apparently free of other problems that could put it at high risk of bias? Additional items included here were therapist qualifications, treatment fidelity, and researcher allegiance or conflict of interest.

A description of what was reported to have happened in each trial was independently extracted by two authors (HT and KS), and a judgement on the risk of bias was made for each source, based on the following three categories.

- Low risk of bias.

- Unclear risk of bias.

- High risk of bias.

Any disagreement was resolved by discussion, or with the help of the third author (SH). For cluster-randomised trials, we had planned to assess risk of bias by considering recruitment bias, baseline imbalance, loss of cluster, incorrect analysis, and comparability with individual randomised trials, in addition to the typical sources. The level of risk of bias was noted in both the body of the review and the 'Summary of findings' table.

\section{Measures of treatment effect}

We used odds ratio (OR) to compare dichotomous data and standardised mean differences (SMD) to analyse continuous data when different scales were used across studies to measure an outcome, and mean difference when the same scale was used across studies or when there was only one study included in a meta-analysis. We considered SMD effect sizes of 0.2 to be small, 0.5 to be medium, and $\geq 0.8$ to be large (Pace 2011). We used a $95 \%$ confidence interval. When an effect was discovered, we had planned to calculate a number needed to treat for an additional beneficial outcome (NNTB) for the primary outcome from the OR, as this value was less likely to be affected by the side (benefit or harm) to which the data were entered (Cates 2002; Deeks 2000; Visual Rx).

We undertook meta-analyses only where this was meaningful, i.e. if the treatments, participants, and the underlying clinical question were similar enough for pooling to make sense. We narratively described skewed data reported as medians and interquartile ranges. Where multiple trial arms were reported in a single trial, we included only the relevant arms.

We combined all types of e-health interventions in the main analyses, and where data allowed, had planned to conduct subgroup analyses to investigate any differences between them.

\section{Unit of analysis issues}

\section{Cluster-randomised trials}

We had planned to include and analyse cluster-randomised trials, as long as proper adjustment for the intra-cluster correlation could be undertaken, as described in the Cochrane Handbook for Systematic Reviews of Interventions (Higgins 2011).

\section{Cross-over trials}

Due to the risk of carry-over effects in cross-over trials, we had planned to only analyse data from the first phase of the trial.

\section{Studies with multiple treatment groups}

Where trials had additional arms that were not e-health interventions, we only included the data relating to the therapy and one control arm in the review. If a trial had more than two arms that met the inclusion criteria, for example two e-health interventions and a control arm, we split data from the control arm equally to produce two (or more) pairwise comparisons.

\section{Dealing with missing data}

We contacted the authors for apparently missing data. We used intention-to-treat (ITT) analysis where this was reported, and mentioned in the 'Risk of bias' table whether or not ITT analysis was done. For continuous data, we used last observation carried forward (LOCF). If necessary, we had planned to conduct a sensitivity analysis to ascertain the effect of multiple missing data management techniques. Where trials did not report the standard deviations (SDs) of continuous measure scores and the original authors were unable to provide them, we calculated the SD from the standard error (SE) or P values (Altman 1996), or from CI, T values, or $\mathrm{P}$ values, as described in section 7.7.3 of the Cochrane Handbook for Systematic Reviews of Interventions (Higgins 2011). If this was not possible, we used the baseline SD. If means were based on imputed data and were all that was available, we used $\mathrm{N}$ - dropout. 


\section{Assessment of heterogeneity}

Before pooling results and carrying out any meta-analysis, we considered clinical heterogeneity and the role of subgroup analyses to address it. We quantified statistical heterogeneity using the $I^{2}$ statistic, with data entered in the way (benefit or harm) that yielded the lowest amount. The amount, depending on the value obtained for the $I^{2}$ statistic (Higgins 2003), was qualified as:

- might not be important (0 to $40 \%$ );

- may represent moderate heterogeneity (30\% to $60 \%$ );

- may represent substantial heterogeneity (50\% to $90 \%$ ); and

- may represent considerable heterogeneity ( $75 \%$ to $100 \%)$.

We took into account (i) magnitude and direction of effects and (ii) strength of evidence for heterogeneity (e.g. the p-value from the chi-squared test, or a confidence interval $(\mathrm{Cl})$ for ${ }^{2}$ ). All heterogeneity was explored, but comparisons with moderate and higher heterogeneity $\left(I^{2}\right.$ statistic $\left.>30 \%\right)$ was further explored using one of the following methods: Egger's regression intercept to assess the possibility of a small trial effect (Rucker 2011); visual forest plot inspection (with trials placed in order according to a specific moderator or subgroup (categorical moderators), or metaregressions (continuous moderators)).

\section{Assessment of reporting biases}

If more than 10 trials were included, we had planned to enter their data into a funnel plot (trial effect versus trial size) in order to evaluate overt publication bias. A symmetrical funnel plot is likely to indicate low publication bias while an asymmetric funnel plot is likely to indicate likely publication bias. The number of trials required to reduce the $P$ value of a statistically significant finding to 0.05 (not statistically significant) is also used to evaluate the robustness of the findings. A high classical fail-safe number indicates that the conclusions are unlikely to be reversed by new trials, while a low classical fail-safe number indicates that they may be more likely to be reversed in the future. Finally, we had planned to use Duval and Tweedie's trim and fill analysis to estimate what the effect size (OR, risk ratio, etc.) would be if there was no publication bias (Duval 2000).

\section{Data synthesis}

When available and sufficiently clinically and statistically homogenous, we combined data from included trials in metaanalyses using the random treatment effects given the expected clinical diversity in the interventions being delivered across various conditions. For consistency we used the random-effects model even when only one trial was included in a meta-analysis; it is also the case that the fixed-effects and random-effects models give the same result when there is only one trial. We presented the characteristics of included and excluded trials in tables. We presented the 'Risk of bias' assessment in a 'Risk of bias' graph. As we were anticipating heterogeneity of data, we had planned to analyse the data in RevMan 5.3 using a random-effects model. We presented results for each comparison as forest plots, when appropriate. We provided narrative summaries for comparisons with fewer than two available trials, and those with a moderate or high level of statistical heterogeneity, following heterogeneity exploration.

\section{Subgroup analysis and investigation of heterogeneity}

For each condition (anxiety or depression), in order to better understand the factors that contributed to an effective intervention, we performed these subgroup analyses for the primary outcomes when there were sufficient trials.

1. Type of experimental therapy (e.g. CBT, other therapy). This was undertaken because different types of therapies are known to have varied underlying theoretical bases and often result in different effect sizes (e.g. Watanabe 2007).

2. Type of control therapy (e.g. active comparators (such as attention placebo, psychological placebo, and other nonpsychological therapies) and non-active comparators (such as treatment as usual and waiting (ist)) as defined by previous researchers (Weisz 2006). Control intervention type has been shown to influence effect sizes (e.g. Furakawa 2014).

3. Modality of delivery (e.g. individual, group). Different modalities of therapy have been shown to result in different effect sizes during the treatment of a range of conditions (Wierzbicki 1987).

4. Dose of treatment (number of completed sessions). Although different therapies will have different total durations, it was of interest to identify therapies that most efficiently resulted in symptomatic improvement.

5. Therapist assistance. There is some evidence that adherence and outcome may be influenced by therapist assistance (Andersson 2009).

6. Form of measurement (e.g. self-rated, parent-rated, clinicianrated). Different types of rating scales have been shown to contribute differently to the prediction of outcomes (Uher 2012).

7. Type of long-term physical conditions (e.g. asthma, diabetes). This was undertaken to identify whether these therapies were more or less effective for children ( 0 to 12 years old) and young people (13 to 18 years old) with different types of physical illness, and in order to make recommendations regarding the targeted use of these therapies.

8. Category of depressive symptoms. There was a possibility that sub-threshold and threshold depressive symptoms may respond differently to therapies (Costello 1992).

9. Target of intervention. Interventions targeted at children or adolescents may be differently effective to those targeted at families (Aydin 2014).

10.Participant factors (e.g. sex, age). Younger and older people have been shown to have different effect sizes following similar therapies, so results were analysed according to four clinicallyrelevant subgroups of age ( 0 to 8,9 to 12,13 to 15 , and 16 to 18 years old (Bennett 2013)).

The feasibility of undertaking these analyses depended upon the number, quality, and heterogeneity of included trials.

\section{Sensitivity analysis}

In order to test the robustness of decisions made during the review process, sensitivity analyses were planned for the primary outcomes only, based on:
1. allocation concealment;
2. dropout rate; and
3. blinding of outcome assessors. 
We had planned to run three separate sensitivity analyses: one where we removed those trials at high or unclear risk of bias for allocation concealment; one where we removed those trials at high or unclear risk of bias for outcome assessor blinding; and one where we removed those trials at high or unclear risk of bias for missing data. We also had planned to run a sensitivity analysis in which we removed those trials where more than $20 \%$ of participants did not complete the post-intervention outcome assessment. The first two have been shown to have the largest impact on treatment effect (Schulz 1995).

\section{'Summary of findings' table}

We constructed a 'Summary of findings' table for each comparison between e-health and any comparator with regard to the following outcomes.

1. Change in severity of anxiety symptoms post-intervention

2. Change in severity of depressive symptoms post-intervention

3. Change in quality of life measures post-intervention

4. Change in functioning measures post-intervention

5. Change in status of long-term physical condition postintervention

In the 'Summary of findings' tables, we used the principles of the GRADE approach to assess the extent to which there could be confidence that the obtained effect estimate reflected the true underlying effect (Guyatt 1998). The quality of a body of evidence was judged on the basis of the included trials' risks of bias, the directness of the evidence, unexplained heterogeneity, imprecision, and the risk of publication bias. A criterion of $<400$ participants for imprecision was used based on Consumer and Communication Cochrane Review Group (Ryan 2016). We used the average rate in all the arms of included trials as the 'assumed risk' for each outcome. As we were not aiming to target any particularly high- or low-risk populations, all the tables were for medium-risk populations. We used GRADEpro GDT to develop the 'Summary of findings' table (GRADEpro GDT).

\section{RES U L T S}

\section{Description of studies}

\section{Results of the search}

We found 2009 citations using the search strategy run between May and August 2016, from which we identified 30 abstracts as potentially relevant. The authors of six trials were contacted for additional information (Aubin 2014; Blackwell 2012; Cheng 2013; Clarke 2015; Ketchen 2006; Quittner 2013). One author reported that their trial had been prematurely discontinued due to lack of funding (Quittner 2013). The ANZCTR record of Clarke 2015 showed that the trial had been discontinued for unspecified reasons, and we received no reply from the other four authors. Following review of the full-text articles, 25 trials were excluded, and five trials were included in the review, each of which contributed data to at least one analysis (Law 2015; Newcombe 2012; Palermo 2009; Palermo 2016a; Trautmann 2010).

CCMD's information specialist ran an update search on 18 August 2017, and retrieved 900 further records (after de-duplication). We screened these and identified no new studies. An updated search of other databases yielded two new study reports, one was excluded
(Starbright programme) and the other was an additional reference to a study already listed as ongoing see Figure 1 for further details.

\section{Included studies}

Five trials were included in this review, with characteristics as follows (see also Characteristics of included studies).

\section{Design}

All five included trials were randomised controlled trials, undertaken between 1997 and 2016. One trial had multiple treatment groups (Trautmann 2010). We did not identify any suitable cluster-randomised or cross-over trials.

\section{Sample sizes}

Sample sizes ranged from 42 (Newcombe 2012), to 273 (Palermo 2016a).

\section{Settings}

Three of the included trials were conducted in the USA (Law 2015; Palermo 2009; Palermo 2016a), one was undertaken in Australia (Newcombe 2012), and one in Germany (Trautmann 2010). Apart from one trial in which a community sample was recruited by advertisements, trials were usually undertaken with outpatients in community clinic settings (Trautmann 2010). These included a neurology clinic (Law 2015, a respiratory clinic (Newcombe 2012), and one or more pain clinics (Palermo 2009; Palermo 2016a). No trials were conducted in inpatient or other settings.

\section{Participants}

Participants were aged between 10 and 18 years. Age ranges in individual trials were as follows: 11 to 17 years, mean 14.5 years (Law 2015); 10 to 17 years, mean 13.5 years (Newcombe 2012); 11 to 17 years, mean 14.8 years (Palermo 2009); 11 to 17 years, mean 14.7 years (Palermo 2016a); and 10 to 18 years, mean 12.7 years (Trautmann 2010). Between $15 \%$ and $50 \%$ of participants were male. The proportion of males in individual trials was as follows: 15\% (Law 2015): 50\% (Newcombe 2012): 30\% (Palermo 2009); 25\% (Palermo 2016a); 45\% (Trautmann 2010). The ethnicity of participants varied between trials. Individual trial demographics were as follows: $92 \%$ White, 3\% Black,5\% Asian, 8\% multi-racial (Law 2015); 100\% White (Newcombe 2012); 90\% Caucasian (Palermo 2009); 85\% Anglo-American, 5\% African American, $1 \%$ Hispanic, 6\% Other, 2\% missing (Palermo 2016a); and unspecified (Trautmann 2010). All participants had a long-term physical condition and symptoms of either anxiety or depression, but none had formal diagnoses of anxiety or depressive disorders. Baseline levels of anxiety were rated as subthreshold in Law 2015, and mild in Palermo 2016a. Baseline levels of depression were as subthreshold in four trials, and mild in Palermo 2016 a.

The main type of long-term physical conditions targeted by identified interventions were pain-related disorders. These included: migraine, tension headache, other headache (Law 2015); chronic idiopathic pain (Palermo 2009): headache, abdominal pain, musculoskeletal pain, other pain (Palermo 2016a); and migraine and tension headache (Trautmann 2010). Only Newcombe 2012 targeted asthma, cystic fibrosis, and other respiratory illness. Three out of five trials that identified severity of long-term physical conditions rated participants as having a mild to moderate level of symptoms. These included having headaches for 6 out of 10 
days and a pain intensity of 4.5 out of 10 (Law 2015); a Forced Expiratory Volume in 1 second (FEV1) of around 75\% (Newcombe 2012); and having headaches for 10.7 days a month and a pain intensity rating of 5.2 out of 10 (Trautmann 2010). Two trials did not report the severity of participants' long-term physical conditions (Palermo 2009; Palermo 2016a). In three of the five included trials, people with medical comorbid conditions were excluded. In Law 2015, young people with developmental disabilities were also excluded and in Palermo 2016a, young people with psychiatric conditions were also excluded. Two authors made no mention of the inclusion or exclusion of young people with comorbid medical or psychological conditions (Newcombe 2012; Trautmann 2010).

Inclusion criteria varied considerably between trials, partly due to the heterogeneity of long-term physical conditions (see Characteristics of included studies for details of individual trials). Exclusion criteria were more consistent, and included the lack of Internet access (Law 2015), difficulties with language (Law 2015; Newcombe 2012; ; Palermo 2009; Palermo 2016a), the inability to use a computer (Newcombe 2012; Palermo 2016a), not residing at home (Palermo 2016a), previous or current use of psychotherapy, especially CBT (Palermo 2009; Trautmann 2010), and recently starting prophylactic medication for headache (Trautmann 2010). Two trials excluded people with serious psychiatric symptoms, but not symptoms of anxiety or depression (Newcombe 2012; Palermo 2016a). Three trials reported there were no pre-treatment differences between groups (Law 2015; Newcombe 2012; Trautmann 2010; ; ). The author of Palermo 2009 reported that their intervention group was slightly, and nonsignificantly, younger than their control group, while the author of Palermo 2016a identified that their intervention group was more likely to be Anglo-American than their control group.

\section{Interventions}

Three of the included trials evaluated the same intervention, namely Web-MAP, a web-based intervention for managing chronic pain (Law 2015; Palermo 2009; Palermo 2016a). The other two trials evaluated an online intervention (Breathe Easier Online) for improving respiratory function (Newcombe 2012), and an online form of multimodal CBT training for reducing headache (Trautmann 2010). All of these interventions were delivered online, and Trautmann 2010 also included a set of relaxation exercises on a computer disc (CD). Two of the three interventions (WebMAP and multimodal CBT training) used CBT as their therapeutic modality. Components of these interventions included education about pain, recognition of stress and negative emotions, deep breathing and relaxation, the implementation of coping skills at school, the development of cognitive skills (e.g. reducing negative thoughts), education about sleep hygiene and lifestyle, activity pacing and scheduling, and relapse prevention. The third intervention (Breathe Easier Online) was based on problem-solving therapy. All were adapted from existing face-to-face individual or group therapies, and were delivered using a manualised format. None of the interventions used biofeedback.

One of the interventions (Web-MAP) included modules for both children and parents, while the other two (Breathe Easier Online and multimodal CBT training) only included modules for children. The duration of interventions was relatively similar. Web-MAP included eight child modules (of 30 minutes each) and eight parent modules (of 30 minutes each) to be completed over an eight-week period; Breathe Easier Online included six child modules (of one hour each) to be completed over a nineweek period; and multimodal CBT training included six child modules (of one hour each) to be completed over an eightweek period. All interventions included some form of homework, usually behavioural assignments, although these were more clearly quantified in trials of Web-MAP (six assignments) than those of the other two interventions. Web-MAP included up to one hour of online coaching (review of assignments and asynchronous feedback) by a post-doctoral fellow or trained therapist. Breathe Easier Online included 'minimal' therapist support (an unquantified amount of troubleshooting and review of assignments). Multimodal CBT training included up to an hour of therapist support (review of assignments and two booster contacts at week four and week eight).

Two trials used attention placebo control conditions. Palermo 2016a used an Internet education programme about chronic pain, with an unspecified number of modules over the same duration as the primary intervention. Trautmann 2010 had two control arms: i) applied relaxation via CD with differential, cue-controlled, and full relaxation procedures delivered in modules over six weeks, with homework exercises and weekly email contact by a therapist; and ii) an educational intervention involving an hourlong online education about chronic headache, and weekly followup email contact by a therapist to check on the maintenance of a headache diary. The educational intervention arm was deemed a more suitable comparator during data analysis, as it included an online component. Two of the trials used treatment as usual as a control intervention. In Law 2015, treatment as usual included a variable number of sessions of psychological therapy (including CBT for pain) or physiotherapy, with or without medication, over the same duration as the primary intervention. In Palermo 2009, treatment as usual included any kind of psychological therapy or waiting list, over the same duration as the primary intervention. Newcombe 2012 used a waiting list control. No trials used psychological placebo or non-psychological therapies as control conditions. Adjunctive treatments were allowed alongside the primary intervention in two trials. Law 2015 allowed medication, psychological therapy (including CBT for pain) and physiotherapy. Palermo 2009 allowed the use of medication and physiotherapy. The use of adjunctive treatment was not described by the other three trials (Trautmann 2010; Newcombe 2012; Palermo 2016a).

\section{Primary outcomes}

Treatment efficacy was evaluated using validated scales that measured changes in the severity of symptoms of either anxiety or depression. A greater number of trials measured changes in depression symptoms than changes in anxiety symptoms. Changes in the severity of anxiety symptoms were measured using the Revised Children's Manifest Anxiety Scale (RCMAS 2) in Law 2015, and the pain-specific anxiety subscale of the Bath Adolescent Pain Questionnaire in Palermo 2016a. Changes in the severity of depression symptoms were measured using the Childhood Depression Inventory in Trautmann 2010 and Law 2015, the Centre for Epidemiological trials Depression scale for children (CES-D C) in Newcombe 2012, the depression subscale of the Revised Child Anxiety and Depression Scale (RCADS) in Palermo 2009, and the depression-specific subscale of the Bath Adolescent Pain Questionnaire in Palermo 2016a.

Treatment acceptability was quantitatively evaluated using validated scales in four trials. These included the Intervention 
Satisfaction Scale (ISS) in Newcombe 2012, the Treatment Evaluation Inventory - Short Form (TEI-SF) in Palermo 2009 and Palermo 2016a, and the Patient Therapist Alliance (PTA) in Trautmann 2010. In addition to these measures, we assessed treatment acceptability based on the number of dropouts and adverse outcomes.

\section{Secondary outcomes}

Changes in 'caseness' (remission or response) of anxiety or depression were not reported by any of the included trials, neither was suicide-related behaviour, defined as the number of a) deaths by suicide, b) suicide attempts, and c) episodes of deliberate selfharm, either reported, or measured using validated scales (Osman 2001). Only Trautmann 2010 measured improvement in quality of life following intervention, using the KINDL-R, a German scale that included six dimensions of the Health-Related Quality of Life Scale. Functioning, as a proxy for psychological well-being, was measured using the Child activity Limitations Interview (CALI) in three trials (Law 2015; Palermo 2009; Palermo 2016a), and the Social ProblemSolving Inventory - Revised (Short Form) in one trial (Newcombe 2012). Status of the long-term physical condition was assessed in Newcombe 2012 with the Forced Expiratory Volume in 1 second (FEV1); in Palermo 2009 and Palermo 2016a with an 11-point pain intensity scale; and in Trautmann 2010, using the frequency of headaches per week recorded in a diary. Adherence to the treatment of the long-term physical condition was not assessed by any of the trials, neither was school or college attendance (e.g. reduction in number of days missed), or economic benefits (e.g. reduction of costs of treatment, number of appointments with general practitioners, use of additional treatments, ability to trial or work).

\section{Excluded studies}

We excluded 25 trials from this review. Seven were excluded as neither changes in anxiety nor changes in depression were measured during these trials (Al-Haggar 2006; Berndt 2014; Fernandes 2015; Hanberger 2013; Newton 2013; Nijhof 2011; Stinson 2010). Eight were excluded as they were identified as not being randomised controlled trials, cluster-randomised controlled trials, or cross-over trials (Blocher 2013; Holden 1999; Holden 2002; Ketchen 2006; Li 2011; Reigada 2013; Seitz 2014; Tung 2015). Seven were excluded as they did not include an e-Health intervention arm (Alemi 2014; Kotses 1991; Liu 2001; Sansom-Daly 2012; Yetwin 2012; Zinchenko 2014; Piaserico 2016). Two trials were excluded as they were not undertaken with children with long-term physical conditions (O'Hea 2013; Pham 2016). One was excluded as it was not an individual trial report (systematic review (Miller 2012)).

\section{Ongoing studies}

Searches to August 2017 identified a total of five ongoing studies. Three trials were reported as ongoing during our original search in July 2016. These included a trial of iACT, an interactive mHealth monitoring system to enhance psychotherapy for adolescents with sickle cell disease (Cheng 2013), a pilot randomised trial of a cognitive behavioural treatment for insomnia and depression in adolescents (Clarke 2015), and a trial of U-care, an internet-based self-help programme of psychosocial support and psychological treatment (Mattson 2013). Although an ANZCTR report stated that one trial had been stopped early, no reply was received from the author when we contacted them for confirmation (Clarke 2015). Two further study protocols were identified during an update of the search in August 2017. These were protocols for a trial of web-based cognitive behavioural therapy for anxiety and depression in youth with chronic illness (Benson 2015), and for a randomised controlled trial of e-Health mindfulness-based intervention versus in-person mindfulness for adolescents with chronic illness (Kaufman 2017). For further details, please see the Characteristics of ongoing studies table.

\section{Studies awaiting classification}

Five studies classed as awaiting classification, as only an abstract with insufficient data was available, despite contacting the authors multiple times (Aubin 2014; Blackwell 2012; Quittner 2013; SansomDaly 2014; Sansom-Daly 2015). For further details, please see the 'Characteristics of studies awaiting classification' table.

\section{Risk of bias in included studies}

For details of the risk of bias judgements for each trial using Cochrane criteria, see Characteristics of included studies. We have presented a graphical representation of the overall risk of bias in included trials in Figure 2 and Figure 3. 
Figure 2. Risk of bias summary: review authors' judgements about each risk of bias item for each included study

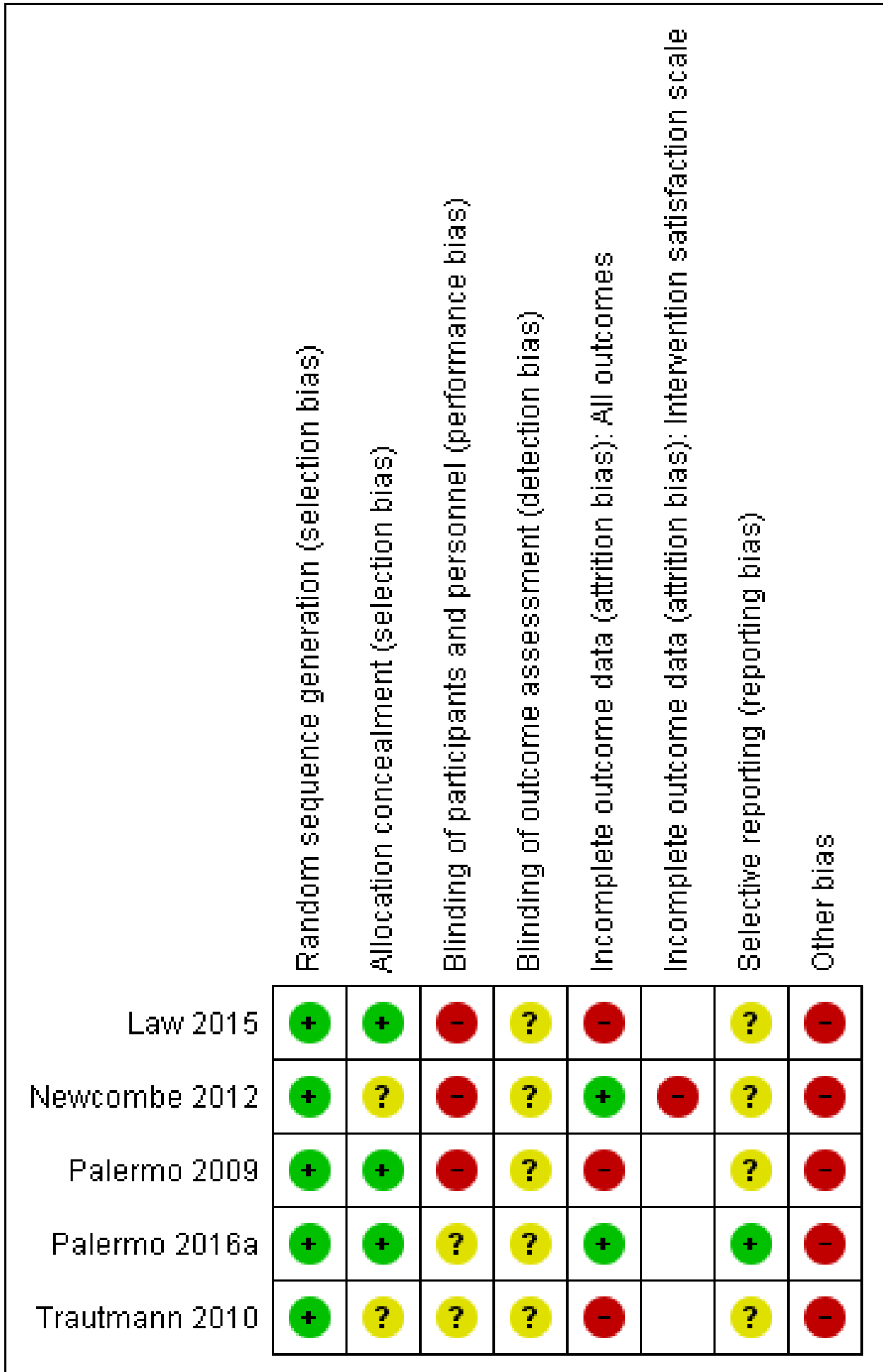

E-Health interventions for anxiety and depression in children and adolescents with long-term physical conditions (Review) 
Figure 3. Risk of bias graph: review authors' judgements about each risk of bias item presented as percentages across all included studies

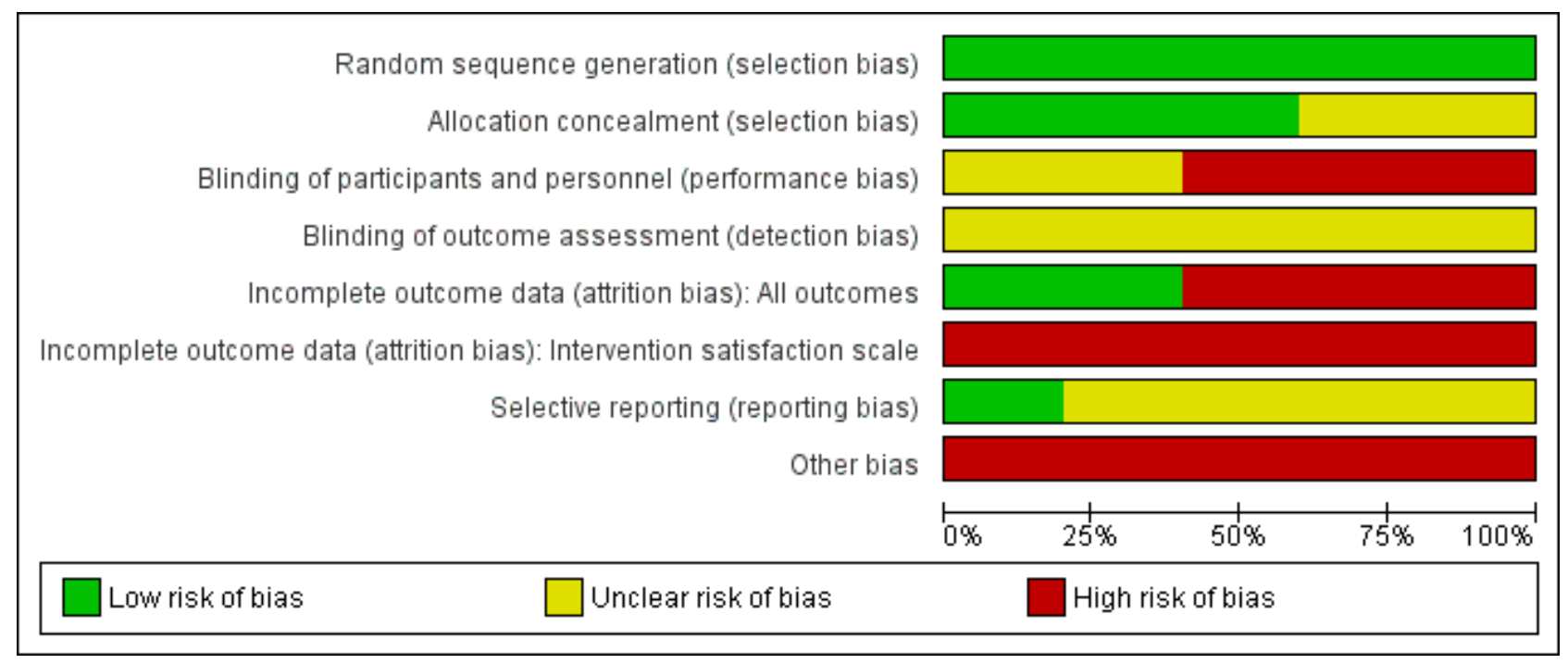

\section{Allocation}

\section{Random sequence generation}

The risk of bias for random sequence generation was considered low in all trials, as online number generation was used, and in most cases (apart from Newcombe 2012), this was associated with block randomisation.

\section{Allocation concealment}

The risk of bias for allocation concealment was considered low in three of the five trials. In these trials, participants had been allocated by one of the following methods: a 1:1 ratio via passwordprotected spreadsheet accessible only to research coordinator (Law 2015); group assignment via identity numbers in sealed envelopes that were only opened by the research coordinator at the end of the trial (Palermo 2009); and pre-programmed assignment within the Web-MAP intervention (Palermo 2016a). In the other two trials, risk of bias for allocation concealment was rated as unclear because the method used was not adequately described (Newcombe 2012; Trautmann 2010).

\section{Blinding}

\section{Participants and personnel}

The risk of bias for blinding of participants and research assistants was rated as unclear or high in all trials. In three trials, these individuals were reportedly not blinded, due to the nature of the intervention (Law 2015; Palermo 2009; Palermo 2016a). In the other two trials, blinding was not clearly described (Newcombe 2012; Trautmann 2010).

\section{Outcome assessors}

The risk of bias for blinding of outcome assessors was rated as unclear in all trials. Outcome measurement was completed online by the participants in two (Law 2015; Palermo 2016a), and blinding was not described in three (Newcombe 2012; Palermo 2009; Trautmann 2010).

\section{Incomplete outcome data}

The risk of bias for incomplete outcome data was rated as low in two trials, as the dropout rates were relatively low and reasons for attrition and exclusion were adequately reported (Newcombe 2012; Palermo 2016a). We rated three trials as having a high risk of bias for incomplete outcome data because inadequate methods were used for ITT analysis (Law 2015; Palermo 2009), or because more than $10 \%$ of post-intervention data was not collected (Law 2015; Trautmann 2010).

\section{Selective reporting}

Only one trial had a full trial protocol that was consistent with the trial report (Palermo 2016b). As none of the other trials had a published trial protocol, these were judged to be of unclear risk of bias.

\section{Other potential sources of bias}

All three interventions tested during the five included trials were conducted by developers of those interventions, so trials were considered at high risk of bias in this regard.

\section{Effects of interventions}

See: Summary of findings for the main comparison EHealth interventions compared to any comparator for anxiety and depression in children and adolescents with long-term physical conditions

Data were only available for some of the primary and secondary outcomes that we had planned to assess. Therefore, we provided a narrative analysis for all outcomes, and a meta-analysis for selected outcomes. Pain was the only type of symptom for the long-term physical conditions for which meta-analysable data were available, so this outcome was labelled 'Pain' in relevant tables. As trials reported pre-intervention and post-intervention scores using different scales for almost all outcomes, standardised mean differences (SMD) were used to pool results in accordance with the recommendation of the Cochrane Handbook for Systematic Reviews of Interventions (Higgins 2011). 


\section{Comparison 1: E-health interventions versus any comparator}

See Summary of findings for the main comparison.

\subsection{Treatment efficacy}

Using GRADE criteria, there was very low-quality evidence from all five trials, involving 441 participants, meaning it is not clear whether there are differences between e-health interventions and comparators in reducing symptoms of depression immediately post-intervention (SMD - $0.06,95 \% \mathrm{Cl}-0.35$ to $0.23 ; \mathrm{I}^{2}=41 \%$; Analysis 1.1 ), or at three to six-month follow-up (SMD $0.04,95 \% \mathrm{Cl}-0.18$ to $0.25 ;\left.\right|^{2}=0 \%$; Analysis 1.2 ).

Two trials, involving 324 participants, measured changes in symptoms of anxiety (Law 2015; Palermo 2016a). These trials offered very low-quality evidence meaning it is not clear whether there are differences between e-health interventions and comparators in reducing symptoms of anxiety either immediately post-intervention (SMD $-0.07,95 \% \mathrm{Cl}-0.29$ to $0.14 ; \mathrm{I}^{2}=0 \%$; Analysis 1.3 ), or three to six months later (SMD $0.02,95 \% \mathrm{Cl}-0.20$ to $0.24 ; \mathrm{I}^{2}$ $=0 \%$; Analysis 1.4).

\subsection{Treatment acceptability}

Treatment acceptability was gauged on the basis of quantitative measures of acceptability, dropouts, and adverse events. Treatment acceptability was quantitatively measured immediately post-intervention in both experimental and control groups by two trials, involving 304 participants (Palermo 2016a; Trautmann 2010). Given the very low-quality evidence from these trials it could not be determined whether e-health interventions were less acceptable to users than any comparator when measured immediately-post intervention (SMD 0.46, 95\% Cl 0.23 to $0.69 ; \mathrm{I}^{2}=0 \%$; Analysis 1.5). Despite this comparative finding, the authors of one trial reported that most children $(S D=1.8)$ and parents $(S D=1.9)$ had good treatment engagement with the intervention, completing a mean of $7 / 8$ modules, and with nobody dropping out of the trial in either group (Palermo 2016a).

One potential treatment-related adverse effect (thoughts of selfharm in response to a behavioural assignment) was identified during this trial (Palermo 2016a). The authors of the other trial did not report any adverse outcomes, but reported that $4 / 24$ (16.6\%) dropped out of the experimental group and 1/19 (5.3\%) dropped out of the control group (Trautmann 2010). Reasons for dropping out included lack of motivation, headache while reading online material, and computer problems. The $\mathrm{Chi}^{2}$ test did not reveal any significant differences in these reasons between experimental and control groups.

\section{Secondary outcomes}

\subsection{Change in caseness (remission or response)}

No data were available for this outcome.

\subsection{Suicide-related behaviour}

No data were available for this outcome.

\subsection{Improvements in quality of life}

Improvements in quality of life were only assessed by one trial, involving 34 participants (Trautmann 2010). This trial provided very low-quality evidence that the e-health intervention was better at improving quality of life than any comparator immediately post- intervention (MD - $0.30,95 \% \mathrm{Cl}-0.54$ to -0.06 ; Analysis 1.6), but not six months after completion of the intervention (MD $0.10,95 \% \mathrm{Cl}$ -0.19 to 0.39 ; Analysis 1.7 ).

\subsection{Functioning}

Changes in functioning were assessed by three trials, involving 372 participants (Law 2015; Palermo 2009; Palermo 2016a). Given the very low-quality evidence it could not be determined whether ehealth interventions were more effective than any comparator in improving functioning, when measured either immediately postintervention (SMD $-0.08,95 \% \mathrm{Cl}-0.33$ to $0.18 ; \mathrm{I}^{2}=17 \%$; Analysis 1.8 ), or three months later (SMD $-0.13,95 \% \mathrm{Cl}-0.35$ to $0.09 ; \mathrm{I}^{2}=0 \%$; Analysis 1.9).

\subsection{Status of the long-term physical condition}

The status of the long-term physical condition was assessed by changes in pain and respiratory function by all five trials, involving 463 participants. Given the low-quality evidence it could not be determined whether e-health interventions were more effective than any comparator in reducing pain immediately postintervention (SMD $0.06,95 \% \mathrm{Cl}-0.12$ to $0.24 ; \mathrm{I}^{2}=0 \%$; Analysis 1.10 ), or three to six months later (SMD $0.10,95 \% \mathrm{Cl}-0.11$ to $0.32 ; I^{2}=0 \%$; Analysis 1.11).

\subsection{Adherence to treatment of long-term physical condition}

No data were available for this outcome.

\subsection{School or college attendance}

No data were available for this outcome.

\subsection{Economic benefits}

No data were available for this outcome.

\section{Comparison 2: E-health interventions versus attention placebo}

Two trials, involving 304 participants, contributed data to this comparison (Palermo 2016a; Trautmann 2010).

\section{Primary outcomes}

\subsection{Treatment efficacy}

There was very low-quality evidence from two trials, involving 304 participants. Therefore, it could not be determined whether ehealth interventions were more effective than attention placebo in reducing symptoms of depression immediately post-intervention (SMD $0.11,95 \% \mathrm{Cl}-0.11$ to $0.34 ; \mathrm{I}^{2}=0 \%$; Analysis 2.1 ) or by threeto six-month follow-up (SMD $0.02,95 \% \mathrm{Cl}-0.21$ to $0.25 ; 1^{2}=0 \%$; Analysis 2.2).

Only one trial, involving 269 participants, measured changes in symptoms of anxiety (Palermo 2016a). There was not enough good quality evidence to determine if there were differences between ehealth interventions and attention placebo in reducing symptoms of anxiety either immediately post-intervention (MD $-0.29,95 \% \mathrm{Cl}$ -1.73 to 1.15; Analysis 2.3), or three to six months later (MD 0.12, $95 \%$ $\mathrm{Cl}-1.27$ to 1.51 ; Analysis 2.4).

\subsection{Treatment acceptability}

Treatment acceptability was quantitatively measured immediately post-intervention in both experimental and control groups by two 
trials involving 304 participants. These trials provided very lowquality evidence that e-health interventions were less acceptable to users than attention placebo (SMD $0.46,95 \% \mathrm{Cl} 0.23$ to $0.69 ; \mathrm{I}^{2}=$ $0 \%$; Analysis 2.5). Potential reasons are explored in the discussion section. Despite this comparative finding, the authors of Palermo 2016a reported that most children $(S D=1.8)$ and parents $(S D=$ 1.9) had good treatment engagement, completing a mean of $7 / 8$ modules, and with nobody dropping out of the trial in either group.

One potential treatment-related adverse effect (thoughts of selfharm in response to a behavioural assignment) was identified during this trial. The authors of Trautmann 2010 did not report any adverse outcomes, but reported that 4/24 (16.6\%) dropped out of the experimental group and 1/19 (5.3\%) dropped out of the control group. Reasons for dropping out included lack of motivation, headache while reading online material, and computer problems. The $\mathrm{Chi}^{2}$ test did not reveal any significant differences in these reasons between experimental and control groups.

\section{Secondary outcomes}

\subsection{Change in caseness (remission or response)}

No data were available for this outcome.

\subsection{Suicide-related behaviour}

No data were available for this outcome.

\subsection{Improvements in quality of life}

Improvements in quality of life were only assessed by one trial, involving 34 participants (Trautmann 2010). This trial provided very low-quality evidence that e-health interventions were better at improving quality of life than attention placebo immediately postintervention (MD $-0.30,95 \% \mathrm{Cl}-0.54$ to -0.06 ; Analysis 2.6), but not six months after completion of the intervention (MD $-0.10,95 \% \mathrm{Cl}$ -0.19 to 0.39 ; Analysis 2.7)

\subsection{Functioning}

Changes in functioning were assessed by one trial involving 269 participants (Palermo 2016a). This trial provided very low-quality evidence, therefore it was not possible to determine whether ehealth interventions were more effective than attention placebo in improving functioning either immediately post-intervention (MD $0.03,95 \% \mathrm{Cl}-1.05$ to 1.11 ; Analysis 2.8 ), or three months later (MD $-0.72,95 \% \mathrm{Cl}-1.84$ to 0.40 ; Analysis 2.9 ).

\subsection{Status of the long-term physical condition}

The status of the long-term physical condition was assessed by changes in pain by two trials involving 302 participants. Together, they provided low-quality evidence meaning it could not be determined whether e-health interventions were more effective than attention placebo in reducing pain immediately postintervention (SMD $0.03,95 \% \mathrm{Cl}-0.34$ to $0.40 ; 1^{2}=32 \%$; Analysis 2.10 ), or three to six months later (SMD $0.13,95 \% \mathrm{Cl}-0.10$ to 0.36 ; Analysis 2.11).

\subsection{Adherence to treatment of long-term physical condition}

No data were available for this outcome.

\subsection{School or college attendance}

No data were available for this outcome.

\subsection{Economic benefits}

No data were available for this outcome.

\section{Comparison 3: E-health interventions versus treatment as usual}

One trial involving 77 participants contributed data to this comparison (Law 2015).

\section{Primary outcomes}

\subsection{Treatment efficacy}

There was very low-quality evidence from one trial, involving 77 participants. Therefore it could not be determined whether ehealth interventions were more effective than treatment as usual in reducing symptoms of depression immediately post-intervention (MD -1.18, 95\% Cl -6.60 to 4.24; Analysis 3.1), or by three- to sixmonth follow-up (MD 1.01, 95\% Cl -3.39 to 5.41; Analysis 3.2).

Similarly, it could not be detemrined whether e-health interventions were more effective than treatment as usual at reducing symptoms of anxiety immediately post-intervention (MD $-1.99,95 \% \mathrm{Cl}-7.31$ to 3.33 ; Analysis 3.3), or by three- to six-month follow-up (MD 0.46, 95\% Cl-5.34 to 6.26; Analysis 3.4).

\subsection{Treatment acceptability}

No data were available for this outcome.

\section{Secondary outcomes}

\subsection{Change in caseness (remission or response)}

No data were available for this outcome.

\subsection{Suicide-related behaviour}

No data were available for this outcome.

\subsection{Improvements in quality of life}

No data were available for this outcome.

\subsection{Functioning}

Changes in functioning were assessed by one trial involving 77 participants. This trial provided very low-quality evidence meaning that it could not be determined whether e-health interventions were more effective than treatment as usual in improving functioning either immediately post-intervention (MD $-0.03,95 \% \mathrm{Cl}-2.56$ to 2.50 ; Analysis 3.5 ), or three to six months later (MD $-0.08,95 \% \mathrm{Cl}-2.76$ to 2.60 ; Analysis 3.6).

\subsection{Status of the long-term physical condition}

The status of the long-term physical condition was assessed by changes in pain by one trial involving 77 participants. This trial provided low-quality evidence meaning that it could not be determined whether e-health interventions were more effective than treatment as usual in reducing pain immediately postintervention (MD $-0.07,95 \% \mathrm{Cl}-1.05$ to 0.91 ; Analysis 3.7), or three to six months later (MD $-0.05,95 \% \mathrm{Cl}-1.34$ to 1.24 ; Analysis 3.8 ).

\subsection{Adherence to treatment of long-term physical condition}

No data were available for this outcome. 


\subsection{School or college attendance}

No data were available for this outcome.

\subsection{Economic benefits}

No data were available for this outcome.

\section{Comparison 4: E-health interventions versus waiting list}

Two trials, involving 87 participants, contributed data to this comparison (Newcombe 2012, Palermo 2009).

\section{Primary outcomes}

\subsection{Treatment efficacy}

There was very low-quality evidence from two trials involving 87 participants. Therefore it could not be determined whether e-health interventions were more effective than waiting list in reducing symptoms of depression immediately post-intervention (SMD -0.40, $95 \% \mathrm{Cl}-0.91$ to $0.11 ; \mathrm{I}^{2}=28 \%$; Analysis 4.1 ).

Neither of these trials assessed the effectiveness of e-health interventions in reducing symptoms of depression at three to six months, nor did they measure symptoms of anxiety immediately post-intervention, or at three to six months.

\subsection{Treatment acceptability}

Neither trial measured treatment acceptability in both the experimental or control groups, therefore, we could not undertake a meta-analysis.

One trial measured treatment acceptability in the experimental group immediately post-intervention using the Intervention Satisfaction Scale (ISS), and its authors reported that 18/19 (95\%) participants said they were happy to do the programme and $15 / 19$ (79\%) thoroughly enjoyed it. Most participants (18/19 (95\%)) said they would recommend the programme to others. Only two participants dropped out of the experimental group and the remainder completed all six modules (Newcombe 2012). The other trial measured treatment acceptability in the experimental group immediately post-intervention using theTreatment Evaluation Inventory - Short Form (TEI-SF), and its authors reported that children and parents in the experimental group reported moderate to high ratings of treatment acceptability (child report mean = $3.55, \mathrm{SD}=0.80$; parent report mean $=3.82, \mathrm{SD}=0.50$ ), and global satisfaction (child report mean $=3.68, \mathrm{SD}=0.84$; parent report mean $=4.09, S D=0.61)$. Child and parent reports were positively correlated $(r=0.50, P=0.02$ and $r=0.53, P=0.01$, respectively). Children completed a mean of 7.11 (SD $=1.86$ ) out of eight modules, and $26 / 48(77 \%)$ completed all eight modules, while parents competed a mean of $6.42(S D=2.24)$ modules, and $14 / 26(54 \%)$ completed all eight modules. The number of modules completed by families was not significantly correlated with immediate posttreatment primary or secondary outcomes, and dropouts were not reported (Palermo 2009).

Neither trial described any adverse events.

\section{Secondary outcomes}

\subsection{Change in caseness (remission or response)}

No data were available for this outcome.

\subsection{Suicide-related behaviour}

No data were available for this outcome.

\subsection{Improvements in quality of life}

No data were available for this outcome.

\subsection{Functioning}

Changes in functioning were assessed by one trial involving 48 participants. This trial provided very low-quality evidence meaning that it could not be determined whether e-health interventions were more effective than a waiting list in improving functioning immediately post-intervention (MD $-3.31,95 \% \mathrm{Cl}-6.90$ to 0.28 ; Analysis 4.2). No data were available regarding changes in functioning at three to six months.

\subsection{Improvement in symptoms of long-term physical condition}

The status of the long-term physical condition, measured by changes in lung function or pain, was assessed by two trials involving 84 participants. These trials provided very low-quality evidence meaning that it could not be determined whether e-health interventions were more effective than a waiting list in reducing symptoms of the long-term physical condition immediately postintervention (SMD $0.08,95 \% \mathrm{Cl}-0.55$ to $0.72 ; \mathrm{I}^{2}=53 \%$; Analysis 4.3). No data were available regarding symptoms of the long-term physical condition at three to six months.

\subsection{Adherence to treatment of long-term physical condition}

No data were available for this outcome.

\subsection{School or college attendance}

No data were available for this outcome.

\subsection{Economic benefits}

No data were available for this outcome.

\section{Comparison 5: E-health interventions versus psychological placebo}

There were no included trials relevant to this comparison.

\section{Comparison 6: E-health interventions versus other non- psychological therapies}

There were no included trials relevant to this comparison.

\section{Subgroup analyses}

We anticipated considerable clinical and methodological heterogeneity in the trials included in this review, so planned a range of subgroup analyses on the type of experimental therapy, type of control therapy, modality of delivery, dose of treatment, therapist assistance, form of measurement, type of long-term physical condition, category of depressive symptoms, target of intervention, and participant factors. However, data were only available to undertake four of these subgroup analyses for the primary outcomes.

\section{Type of experimental therapy}

Included in the analysis were four trials involving 402 participants who had undertaken cognitive behaviour therapy (CBT) and one trial of 39 participants who had undertaken a non-CBT intervention (Newcombe 2012). Results indicated that the type of experimental 
therapy did make a difference to the change in symptoms of depression immediately post-intervention $\left(\mathrm{Chi}^{2}=4.55, \mathrm{df}=1\right.$ $(P=0.03), I^{2}=78 \%$; Analysis 5.1$)$, however due to the small number of studies, we do not think this finding is reliable. No data were available to comment on the association between the type of experimental therapy and later changes in symptoms of depression, immediate changes in symptoms of anxiety, later changes in symptoms of anxiety or treatment acceptability.

\section{Type of comparator}

Included in the analysis were two trials involving 153 participants who had received attention placebo (Palermo 2016a; Trautmann 2010), one trial involving 23 participants who had received treatment as usual (Law 2015), and two trials involving 42 participants who had received waiting-list control interventions (Newcombe 2012; Palermo 2009). Results indicated that the type of comparator did not make any difference to the change in symptoms of depression immediately post-intervention $\left(\mathrm{Chi}^{2}=3.53, \mathrm{df}=2(\mathrm{P}\right.$ $=0.17), \mathrm{I}^{2}=43.3 \%$; Analysis 6.1 ), change in symptoms of depression three to six months later $\left(\mathrm{Chi}^{2}=0.10, \mathrm{df}=1(\mathrm{P}=0.75), \mathrm{I}^{2}=0 \%\right.$; Analysis 6.2 ), change in symptoms of anxiety immediately postintervention $\left(\mathrm{Chi}^{2}=0.26, \mathrm{df}=1(\mathrm{P}=0.61), \mathrm{I}^{2}=0 \%\right.$; Analysis 6.3), or change in symptoms of anxiety three to six months later $\left(\mathrm{Chi}^{2}=0.01\right.$, $\mathrm{df}=1(\mathrm{P}=0.94), \mathrm{I}^{2}=0 \%$; Analysis 6.4).

\section{Type of long-term physical condition}

Included in the analysis were four trials involving 402 participants who had pain related conditions (Law 2015; Palermo 2009; Palermo 2016a; Trautmann 2010), and one trial of 39 participants who had non-pain-related conditions (Newcombe 2012). Results indicated that the type of long-term physical condition did make a difference in the change in symptoms of depression immediately postintervention $\left(\mathrm{Chi}^{2}=4.56, \mathrm{df}=1(\mathrm{P}=0.03), \mathrm{I}^{2}=78.1 \%\right.$; Analysis 7.1$)$, however, due to the small number of studies, we do not think this finding is reliable.

No data were available to comment on the association between the type of long-term physical condition and later changes in symptoms of depression, immediate changes in symptoms of anxiety, later changes in symptoms of anxiety, or treatment acceptability.

\section{Target of intervention: Child and parent versus child only}

Included in the analysis were three trials involving 367 participants, in which experimental interventions were targeted at parents and children (Law 2015; Palermo 2009; Palermo 2016a), and two trials involving 74 participants, in which experimental interventions were only targeted at children (Newcombe 2012; Trautmann 2010). Results indicated that the target of intervention did not make any difference to the change in symptoms of depression immediately post-intervention $\left(\mathrm{Chi}^{2}=0.09, \mathrm{df}=1(\mathrm{P}=0.76), \mathrm{I}^{2}=0 \%\right.$; Analysis 8.1).

No data were available to comment on the association between the target of intervention and later changes in symptoms of depression, immediate changes in symptoms of anxiety, later changes in symptoms of anxiety or treatment acceptability.

\section{Sensitivity analyses}

Due to the limited number of identified trials, we did not undertake planned sensitivity analyses of the primary outcomes on the bases of allocation concealment, blinding of outcome assessors, or dropout rates.

\section{Reporting Bias}

Given that this review explored a relatively new area of research, we aimed to minimise reporting bias by undertaking comprehensive searches of key databases and other sources of technological reporting. Due to the limited number of included trials and known association of SMDs with a degree of standard error, inspection of funnel plots for the primary outcome measures was of limited value in informing us about the likely presence of publication bias (Higgins 2011). We did not identify any other obvious sources of reporting bias during the review process.

\section{DISCUSSION}

\section{Summary of main results}

The primary objectives of this review were to evaluate the treatment efficacy and acceptability of e-health interventions for treating anxiety and depression in children with long-term physical conditions. From the limited data available, we concluded that the current evidence failed to clearly demonstrate that existing e-health interventions were better than any type of comparator at reducing symptoms of anxiety or depression in this audience. Although there was qualitative data to suggest the acceptability of this treatment modality to this audience, quantitative measures of treatment acceptability suggested that existing e-health interventions may, in some cases, be less acceptable than attention placebo interventions. This may be explained by the extra therapeutic demands, such as the completion of cognitive behavioural therapy (CBT) exercises and homework associated with active interventions.

Secondary aims of this review included identifying changes in caseness of anxiety or depression, quality of life, status of long-term physical conditions, adherence to treatment of longterm physical conditions, functioning, quality of life, school or college attendance, and economic benefits associated with the use of e-health interventions. Data were only available for some of these outcomes. Despite being primarily designed to improve the status of long-term physical conditions, there was not enought high quality evidence to determine whether the ehealth interventions included in this review were better than any type of placebo intervention at improving symptoms of long-term physical conditions. Similarly, there was not enough evidence to determine whether they were clearly better than any type of placebo intervention at improving functioning, or quality of life, suggesting room for the development of more efficacious e-health interventions for this clinical population. Our main findings are summarised in the Summary of findings for the main comparison.

Subgroup analysis was only feasible for four factors, namely, the type of intervention, type of comparator, type of long-term physical condition, and target audience for the intervention. Within the limited selection of trials, there was no evidence of a clear impact of any of these factors on the size of the treatment effect.

\section{Overall completeness and applicability of evidence}

We identified a very limited number of trials from which to draw conclusions, and the limitation of the review to randomised controlled trials may have led to the exclusion of data from quasi- 
randomised and non-randomised trials of e-health interventions for treating anxiety or depression in the target population. Most trials were undertaken with adolescents, making it hard to comment on the effectiveness of these interventions on children younger than 10 years of age, or the value of parental involvement in their use, particularly for younger children. Despite appreciating that the intellectual, emotional, and psychological maturity of young people changes so significantly over even a few years, we had hoped to subgroup our findings by age band, but were unable to do so (Petersen 1995). All included trials were conducted in high income countries, making it similarly impossible to determine the likely effectiveness of these interventions for children and adolescents living in lower income countries, who might have greater need for such seemingly cost-effective therapies. Most included trials were undertaken with adolescents with chronic pain conditions, making it imprudent to generalise findings to children and adolescents with other long-term physical conditions. Most included interventions were also designed to detect differences in symptoms of the long-term physical health condition (four trials), or functioning (one trial), rather than anxiety or depression, rendering them less likely to achieve a reduction in anxiety or depression symptoms. Most participants in these studies had subthreshold levels of anxiety or depression, and it is possible that the effect of these interventions on these conditions may have been masked by a floor effect. Only one trial was specifically powered to detect an improvement in psychosocial well-being (Newcombe 2012).

The five included trials tested only three interventions, namely Web-MAP, which is a CBT-based treatment for adolescent pain; Breathe Easier Online, a problem-solving intervention adapted for children with respiratory conditions; and a multi-modal CBTbased training programme for children with recurrent headache. This reflects the newness of the field of e-health research, and confirms the fact that there is significant room for the development of other e-health interventions. A variety of control interventions were used in the included trials. Only two trials tested e-health interventions against the most stringent type of comparator, an attention placebo. Outcome measurement was generally short-term in nature, immediately post-intervention and three to six months later. No trials measured longer-term outcomes. Significant markers of treatment effectiveness, such as suiciderelated behaviour, adherence to treatment of long-term physical conditions, and markers of functional and social impact, such as school attendance and economic benefits were not collected during any of the identified trials, making it difficult to appreciate the wider implications of these interventions.

\section{Quality of the evidence}

The quality of the evidence for all outcomes for which there were data was low or very low quality. With the exception of randomisation, we assessed almost all domains to be of unclear or high risk of bias across (Figure 2; Figure 3). None of the included trials were designed to investigate the effect of e-health interventions on anxiety or depression. There was considerable heterogeneity of results as evident from $\mathrm{I}^{2}$ results, magnitude, and the bidirectional nature of effect. Only one trial had an available trial protocol, making the others at unclear risk of selective reporting. As there were not enough trials to conduct funnel plot evaluation, we were unable to be certain there was no publication bias.

\section{Potential biases in the review process}

None of the review authors were involved in any of the trials, however $\mathrm{HT}, \mathrm{KS}, \mathrm{SM}$, and $\mathrm{SH}$ are all involved in the development of e-health interventions for treating psychological problems in children and adolescents. Previous reviewers have drawn attention to the 'file drawer' effect of smaller trials with negative short-term results, and despite conducting as thorough a search as we could of key databases, trial registries, and other sources, we may have missed some trials of existing e-health interventions (Sansom-Daly 2014). However, it is likely that the results of this review reflected a genuine lack of e-health interventions designed to treat anxiety and depression in children and adolescents with long-term physical conditions.

\section{Agreements and disagreements with other studies or reviews}

This review addresses novel areas of practice and research, therefore, the limited number of eligible trials is not surprising. It echoes the sparse findings of three recent and related systematic reviews. The first reviewed mobile phone messaging to facilitate self-management of long-term illnesses, and only identified four interventions, with limited evidence of efficacy (de Jongh 2012). The second reviewed psychological interventions for depression in adolescents and adults with congenital heart disease, did not find any trials that met the criteria for analysis (Lane 2013). The third reviewed psychological interventions for mental health disorders in children with chronic illness (Bennett 2015). They identified only ten trials, including one trial of computerised CBT for anxiety and depression, in children and adolescents with epilepsy (Blocher 2013). Similar to the studies included in our reviews, this non-randomised, single arm pilot trial demonstrated preliminary evidence of the intervention's efficacy, without any adverse effects. The variability in the type of comparator in our review was consistent with previous reviews of related areas, including a systematic review of psychological therapies for the management of chronic and recurrent pain in children and adolescents (Eccleston 2014). Like these reviewers, we found it difficult to be certain how much of the efficacy of interventions was related to the interventions themselves versus other factors, such as clinician attention.

Due to the small number of included trials and no evidence of clear improvement in anxiety or depression, it was not possible to identify specific features of e-health interventions that might improve outcomes of interest. Our subgroup analysis of CBT and non-CBT-based interventions did not show any discernible difference between these therapies. This is in contrast to a previous systematic review of psychological interventions for parents of children and adolescents with chronic illness that showed that CBT was more beneficial for reducing children's primary symptoms, while problem-solving therapy that included parents improved parental adaptive behaviour and parental mental health (Eccleston 2015). All of the included therapies were derived from existing models of psychotherapy, and it is likely that further experimentation with the delivery of such models online will need to continue to achieve the ideal design. 


\section{AUTHORS' CONCLUSIONS}

\section{Implications for practice}

The effects of e-health interventions for treating anxiety or depression in children and adolescents with long-term physical conditions are uncertain, due to very low-quality evidence from a small number of trials, the lack of trials with participants under 10 years of age, and the absence of participants with significant symptoms of anxiety or depression.

Given the global improvement in access to technology (Internet World Stats 2017), the interest and enthusiasm of this population in having access to e-health interventions to support their psychosocial welfare (Thabrew 2016), and the limited number of available interventions, we believe there is room for development of more technologically-based treatments specifically designed to address the needs of children and adolescents with long-term physical conditions. However, due to the heterogeneity of risk factors and precipitants for anxiety and depression, it is unrealistic to expect a single intervention to work for everyone (Hetrick 2016). For the moment, it seems reasonable to treat anxiety and depression in children and adolescents with long-term physical conditions with either face-to face-interventions that have been shown to be effective in this clinical population, or e-health interventions that have been demonstrated to be effective for the management of depression or anxiety more generally in children and adolescents.

\section{Implications for research}

We offer a number of recommendations for future research in this area. All trials should use validated measures of anxiety or depression, and be reported according to CONSORT guidelines in order to ensure the availability of comparable datasets (Schulz 2010). Where possible, attention control groups should be used to better distinguish the specific effects of interventions from the generic benefit of receiving therapeutic attention. Following the successful completion of pilot trials, larger, and better designed trials of e-health interventions should be undertaken, ideally by researchers not directly involved in the development of these interventions, to provide greater certainty of effectiveness. One way of achieving this is by multi-site collaboration. Short-term proof of effectiveness should be followed by trials investigating longerterm effectiveness, and functional and economic benefits. Data on adverse effects of psychotherapeutic interventions should be collected to balance their potential benefits with potential harms.

Larger trials should stratify participants by age group, as there may be developmentally-related differences in effectiveness between children, younger adolescents, older adolescents, and adults. Specific research is needed into the treatment of anxiety and depression in children, as existing interventions have been evaluated mainly with adolescents. Given the likely association between medical illness or treatment and anxiety, research is also needed into interventions designed to address specific healthrelated anxieties. Stratification of participants by type of long-term physical condition, and degree of baseline symptoms of anxiety or depression (subthreshold vs threshold) would also provide an indication of who responds best to different types or features of interventions. Inclusion of participants and research venues in lower and middle income countries and different cultures would be beneficial to evaluate the effectiveness of interventions that could potentially be dispersed globally, to areas of need. Co-design of interventions with participants is further likely to improve their acceptability to target audiences (Orlowski 2015).

As mentioned by previous authors, it is hard to be certain that recipients are actually acquiring or mastering the skills learnt via e-health interventions (Weersing 2009). Analysis of therapeutic design features, including the number and type of components, duration of treatment, parent involvement, therapist involvement, and individual skills should be carried out to identify 'active ingredients'. Due to recognised challenges in delivering software on multiple devices and platforms, and the potential for poor adherence to e-health interventions, research into the delivery and uptake of e-health interventions is needed to ensure that effective interventions are accessible and scalable to larger audiences (Christiensen 2009).

Finally, future updates of this review should report on adherence to these recommendations.

\section{ACKNOWLEDGEMENTS}

The authors acknowledge the valuable contributions of the Cochrane Common Mental Disorders Group (CCMDG), including Sarah Dawson (Information Specialist), Jessica Sharp (Managing Editor), and Rachel Churchill (Co-ordinating Editor). We are also very grateful to Anne Wilson from the Philson Library at the University of Auckland for assisting us with some of the search processes.

This review was supported by funding from the Starship Foundation and Oakley Foundation in New Zealand.

\section{Cochrane Group funding acknowledgement}

The National Institute for Health Research (NIHR) is the largest single funder of the Cochrane Common Mental Disorders Group.

\section{Disclaimer}

The views and opinions expressed herein are those of the authors and do not necessarily reflect those of the NIHR, National Health Service (NHS), or the Department of Health. 


\section{RE F E R E N C E S}

\section{References to studies included in this review}

Law 2015 \{published data only\}

Law EF, Beals-Erickson SE, Noel M, Claar R, Palermo TM. Pilot randomized controlled trial of Internet-delivered cognitivebehavioral treatment for pediatric headache. Headache 2015;55(10):1410-25. [DOI: 10.1111/head.12635]

\section{Newcombe 2012 \{published data only\}}

Newcombe PA, Dunn TL, Casey LM, Sheffield JK, Petsky H, Anderson-James S, Chang AB. Breathe Easier Online: Evaluation of a Randomized Controlled Pilot Trial of an Internet-Based Intervention to Improve Well-being in Children and Adolescents With a Chronic Respiratory Condition. Journal of Medical Internet Research 2012;14(1):e23: 1-12. [DOI: 10.2196/jmir.1997]

\section{Palermo 2009 \{published data only\}}

Palermo TM, Wilson AC, Peters M, Lewandowski A, Somhegyi H. Randomized controlled trial of an Internet-delivered family cognitive-behavioral therapy intervention for children and adolescents with chronic pain. Pain 2009;146(1-2):205-13. [DOI: 10.1016/j.pain.2009.07.034]

\section{Palermo 2016a \{published data only\}}

Palermo TM, Law EF, Fales J, Bromberg MH, Jessen-Fiddick T, Tai G. Internet-delivered cognitive-behavioral treatment for adolescents with chronic pain and their parents: a randomized controlled multicenter trial. Pain 2016;157(1):174-85. [DOI: 10.1097/j.pain.0000000000000348]

\section{Trautmann 2010 \{published data only\}}

Trautmann E, Kroner-Herwi, B. A randomized controlled trial of Internet-based self-help training for recurrent headache in childhood and adolescence. Behaviour Research and Therapy 2010;48(1):28-37. [DOI: 10.1016/j.brat.2009.09.004]

\section{References to studies excluded from this review}

\section{Alemi 2014 \{published data only\}}

Alemi M, Meghardi A, Ghanbarzadeh A, Moghadam JL, Ghanbarzadeh A. Impact of a Social Humanoid Robot as a Therapy Assistant in Children Cancer Treatment: International Conference on Social Robotics ICSR 2014. Social Robotics. 2014:11-22.

\section{Al-Haggar 2006 \{published data only\}}

Al-Haggar Ms, Al-Naggar Za, Abdel-Salam Ma. Biofeedback and cognitive behavioral therapy for Egyptian adolescents suffering from chronic fatigue syndrome. Journal of pediatric neurology 2006;4:161-169.

\section{Berndt 2014 \{published data only\}}

Berndt R-D, Takenga C, Preik P, Kuehn S, Berndt L, Mayer H, Kaps A, Schiel R. Impact of information technology on the therapy of type-1 diabetes: A case study of children and adolescents in Germany. Journal of personalized medicine 2014;4:200-217.
Blocher 2013 \{published data only\}

Blocher JB, Fujikawa M, Sung C, Jackson DC, Jones JE. Computer-assisted cognitive behavioral therapy for children with epilepsy and anxiety: A pilot study. Epilepsy and Behavior 2013;27(1):70-76.

\section{Fernandes 2015 \{published data only\}}

Fernandes S, Liamputtong P, Wollersheim D. What makes people sick? Burmese refugee children's perceptions of health and illness. Health Promotion International 2015;40(1):23-32.

\section{Hanberger 2013 \{published data only\}}

Hanberger L, Ludvigsson J, Nordfeldt S. Use of a web 2.0 portal to improve education and communication in young patients with families: randomized controlled trial. Journal of medical Internet research 2013;15:e175.

Holden 1999 \{published data only\}

Holden G, Bearison DJ, Rode DC, Rosenberg G, Fishman M. Evaluating the effects of a virtual environment (STARBRIGHT World) with hospitalized children. Research on Social Work Practice 1999;9(3):365-382.

\section{Holden 2002 \{published data only\}}

Holden G, Bearison DJ, Rode DC, Kapiloff MF, Rosenberg G, Rosenzweig J. The impact of a computer network on pediatric pain and anxiety: A randomized controlled clinical trial. Social Work in Health Care 2002;36(2):21-33.

\section{Ketchen 2006 \{published data only\}}

Ketchen B, Hazzard A, Lassiter S, Barber N, Lisa Armistead L, Mentz R. STARBRIGHT world: A pilot study of a home-based sickle cell psychoeducational intervention. Childrens Health Care 2006;35(4):321-38.

\section{Kotses 1991 \{published data only\}}

Kotses H, Harver A, Segreto J, Glaus JD, Creer TI, Young GA. Long-term effects of biofeedback-induced facial relaxation on measures of asthma severity in children. Biofeedback and SelfRegulation 1991;16(1):1-21.

\section{Li 2011 \{published data only\}}

Li WH, Chung JO, Ho EK, Chiu SY. Effectiveness and feasibility of using the computerized interactive virtual space in reducing depressive symptoms of Hong Kong Chinese children hospitalized with cancer. Journal for specialists in pediatric nursing 2011;16:190-198.

\section{Liu 2001 \{published data only\}}

Liu C. Can asthma education improve clinical outcomes? An evaluation of a pediatric asthma education program. Journal of Asthma 2001;38(3):269-278.

Miller 2012 \{published data only\}

Miller LD, Silva S, Bouchard S, Belanger C, Taucer-Samson T, Davis TE, Ollendick TH, Ost LG. Using Virtual Reality and Other Computer Technologies to Implement Cognitive-Behavior Therapy for the Treatment of Anxiety Disorders in Youth. 
Intensive One-Session Treatment of Specific Phobias. Vol. 1, New York: Springer, 2012:227-251.

\section{Newton 2013 \{published data only\}}

Newton KT, Ashley A. Pilot study of a web-based intervention for adolescents with type 1 diabetes. Journal of telemedicine and telecare 2013;19:443-449.

\section{Nijhof 2011 \{published data only\}}

Nijhof SL, Bleijenberg G, Uiterwaal Cspm, Kimpen JLL, van de Putte EM. Fatigue In Teenagers on the interNET - The FITNET Trial. A randomized clinical trial of web-based cognitive behavioural therapy for adolescents with chronic fatigue syndrome: study protocol. ISRCTN59878666Bmc. Neurology 2011;11:23.

\section{O'Hea 2013 \{published data only\}}

O'Hea EL, Cutillo A, Harralson T, Grissom G, Person S, Boudreaux ED. Randomized control trial to test a computerized psychosocial cancer assessment and referral program: Methods and research design. Contemporary Clinical Trials 2013;35:15-24.

\section{Pham 2016 \{published data only\}}

Pham Q, Khatib Y, Stansfeld S, Fox S, Green T. Feasibility and Efficacy of an mHealth Game for Managing Anxiety: "Flowy" Randomized Controlled Pilot Trial and Design Evaluation. Games for Health Journal 2016;5(1):50-67.

\section{Piaserico 2016 \{published data only\}}

Piaserico S, Marinello E, Dessi A, Linder MD, Coccarielli D, Peserico A. Efficacy of Biofeedback and Cognitive-behavioural Therapy in Psoriatic Patients. Acta Dermato-Venereologica 2016;96:91-95.

\section{Reigada 2013 \{published data only\}}

Reigada LC, Benkov KJ, Bruzzese JM, Hoogendoorn C, Szigethy E, Briggie A, Walder D J, Warner CM. Integrating illness concerns into cognitive behavioral therapy for children and adolescents with inflammatory bowel disease and cooccurring anxiety. Journal for Specialists in Pediatric Nursing 2013;18(2):133-143.

\section{Sansom-Daly 2012 \{published data only\}}

Sansom-Daly UM, Wakefield CE, Bryant RA, Butow P, Sawyer S, Patterson P, Anazodo A, Thompson K, Cohn RJ. Online groupbased cognitive-behavioural therapy for adolescents and young adults after cancer treatment: A multicenter randomised controlled trial of Recapture Life-AYA. Bmc Cancer 2012;12:339.

\section{Seitz 2014 \{published data only\}}

Seitz DCM, Knaevelsrud C, Duran G, Waadt S, Loos S, Goldbeck L. Efficacy of an internet-based cognitive-behavioral intervention for long-term survivors of pediatric cancer: a pilot study. Supportive Care in Cancer 2014;22(8):2075-2083.

\section{Stinson 2010 \{published data only\}}

Stinson JN, McGrath PJ, Hodnett ED, Feldman BM, Duffy CM, Huber AM, Tucker LB, Hetherington CR, Tse SML, Spiegel LR, Campillo S, Gill NK, White ME. An Internet-based Selfmanagement Program with Telephone Support for Adolescents with Arthritis: A Pilot Randomized Controlled Trial. Journal of Rheumatology 2010;37(9):1944-1952.

Tung 2015 \{published data only\}

Tung J, Grunow JE, Jacobs N. Pilot Development of an Electronic Pediatric Inflammatory Bowel Disease Quiz Game. Journal of Pediatric Gastroenterology and Nutrition 2015;61(3):292-296.

\section{Yetwin 2012 \{published data only\}}

Yetwin A, Marks K, Bell T, Gold J. Heart rate variability biofeedback therapy for children and adolescents with chronic pain. Journal of pain (abstracts of the 31st annual scientific meeting of the american pain society). Honolulu, Hawaii, United States, 2012.

\section{Zinchenko 2014 \{published data only\}}

Zinchenko M, Grishin O, Grishin V. Effectiveness of baseline therapy and respiratory biofeedback in children with asthma and psychogenic dyspnea. European respiratory journal 2014;44:1160.

\section{References to studies awaiting assessment}

Aubin 2014 \{published data only\}

Aubin HJ, Karila L, Reynaud M. Pharmacotherapy for Smoking Cessation: Present and Future. Current Pharmaceutical Design 2014;17(14):1343-1350.

\section{Blackwell 2012 \{published data only\}}

Blackwell LS, Romero SL, Romero CV, McLean KA, Dawkins K, Hoag J, Schoumacher R, Stokes DC. CFfone: A social networking site for adolescents and young adults with cf. Pediatric Pulmonology 2012;47:430.

\section{Quittner 2013 \{published data only\}}

Quittner AL, Romero SL, Blackwell LS, et al. Preliminary data on the efficacy of an online social network for adolescents with CF. American journal of respiratory and critical care medicine 2013;187:A5036.

\section{Sansom-Daly 2014 \{published data only\}}

Sansom-Daly Um, Wakefield Ce, Bryant Ra, Ellis S, Doolan E, Cohn RJ. Adapting evidence-based psychological therapy to the computer screen for adolescent and young adult cancer survivors: Preliminary results from the 'recapture life' randomised controlled trial. Asia-Pacific journal of clinical oncology. Melbourne, Australia, 2014.

\section{Sansom-Daly 2015 \{published data only\}}

Sansom-Daly U, Wakefield C, McGill B, Wilson H, Robertson E, Ellis S, Doolan E, Patterson P, Cohn R. Clinical challenges in implementing psychosocial support in the online space: Experiences from the 'Recapture Life' trial. Psycho-oncology 2015;24:14. 


\section{References to ongoing studies}

Benson 2015 \{unpublished data only\}

NCT02453880. Web-based CBT for symptoms of mild-tomoderate anxiety and depression in youth with chronic illness. clinicaltrials.gov/ct2/show/NCT02453880 (first submitted 27 May 2015).

\section{Cheng 2013 \{published data only\}}

Cheng CW, Brown RC, Cohen LL, et al. ACT - An Interactive mHealth Monitoring System to Enhance Psychotherapy for Adolescents with Sickle Cell Disease. 35th Annual International Conference of the IEEE Engineering in Medicine and Biology Society. Osaka, JAPAN: IEEE Engn Med Biol Soc, Japanese Soc Med \& Biol Engn, 2013.

\section{Clarke 2015 \{published data only\}}

Clarke J, Vatiliotis V, Verge CF, Holmes-Walker J, Campbell LV, Wilhelm $\mathrm{K}$, et al. A mobile phone and web-based intervention for improving mental well-being in young people with type 1 diabetes: design of a randomized controlled trial. Journal of Medical Internet Research 2015;4(2):e50.

\section{Kaufman 2017 \{unpublished data only\}}

NCT03067207. In-Person vs e-Health Mindfulness-Based Intervention for Adolescents With Chronic Illness. https:// clinicaltrials.gov/ct2/show/NCT03067207 2017 [Accessed 19 July 2018].

\section{Mattson 2013 \{published data only\}}

Ander M, Von Essen L, Ljungman G, Mattsson E, Toft T, Norberg Al. U-care: Youngcan-development of an internetbased self-help program of psychosocial support and psychological treatment. Psycho-oncology 2013;22:244.

Mattson S, Alfonsson S, Carlsson M, Nguyen P, Olsson E, Johansson B. U-Care: Internet-based stepped care with interactive support and cognitive behavioral therapy for reduction of anxiety and depressive symptoms in cancer clinical trial protocol. BMC Cancer 2013;13:414.

\section{Additional references}

\section{Abramson 1978}

Abramson LY, Seligman MEP, Teasdale I. Learned helplessness in humans: critique and reformulation. Journal of Abnormal Psychology 1978;87(1):49-59.

\section{Altman 1996}

Altman DG, Bland JM. Detecting skewness from summary information. BMJ 1996;313(7066):1200.

\section{American Psychological Association 2013}

American Psychiatric Association. Diagnostic and Statistical Manual of Mental Disorders. 5th Edition. Arlington (VA): American Psychiatric Publishing, 2013.

\section{Amon 2008}

Amon KL, Campbell A. Can children with AD/HD learn relaxation and breathing techniques through biofeedback video games?.
Australian Journal of Educational \& Developmental Psychology 2008;8:72-84

\section{Andersson 2009}

Andersson G. Using the Internet to provide cognitive behaviour therapy. Behaviour Research and Therapy 2009;47:175-80.

\section{Aydin 2014}

Aydin A. Parental involvement in cognitive-behavioral therapy for children with anxiety disorders. Turk Psikiyatri Dergisi [Turkish Journal of Psychiatry] 2014;25(3):181-9.

\section{Barak 2008}

Barak A, Hen L, Boniel-Nissim M, Shapira N. A comprehensive review and a meta-analysis of the effectiveness of Internetbased psychotherapeutic interventions. Journal of Technology in Human Services 2008;26(2):109-60.

\section{Bateman 2000}

Bateman A, Brown D, Pedder J. Introduction to Psychotherapy: An Outline of Psychodynamic Principles and Practice. 3rd Edition. London (UK): Routledge, 2000.

\section{Beck 1976}

Beck AT. Cognitive Therapy and the Emotional Disorders. New York (NY): International Universities Press, 1976.

\section{Beidel 2000}

Beidel DC, Turner SM, Hamlin K, Morris TL. The Social Phobia and Anxiety Inventory for Children (SPAI-C): external and discriminative validity. Behavior Therapy 2000;31:75-87.

\section{Bennett 2013}

Bennett K, Manassis K, Walter SD, Cheung A, Wilansky-Traynor P, Diaz-Granados N, et al. Cognitive behavioral therapy age effects in child and adolescent anxiety: an individual patient data meta-analysis. Depression and Anxiety 2013;30(9):829-41.

\section{Bennett 2015}

Bennett S, Shafran R, Coughtrey A, Walker S, Heyman I. Psychological interventions for mental health disorders in children with chronic physical illness: a systematic review. Archives of Diseases of Childhood 2015;100(4):308-16.

\section{Benton 2007}

Benton TD, Ifeagwu JA, Smith-Whitley K. Anxiety and depression in children and adolescents with sickle cell disease. Current Psychiatry Report 2007;28:185-90.

\section{Birmaher 1996a}

Birmaher B, Ryan N, Williamson D, Brent D, Kaufman J, Dahl R, et al. Childhood and adolescent depression: a review of the past 10 years. Part I. Journal of the American Academy of Child and Adolescent Psychiatry 1996;35(11):1427-39.

\section{Birmaher 1996b}

Birmaher B, Ryan N, Williamson D, Brent D. Childhood and adolescent depression: a review of the past 10 years. Part II. Journal of the American Academy of Child and Adolescent Psychiatry 1996;35(12):1575-83. 


\section{Birmaher 1999}

Birmaher B, Brent DA, Chiappetta L, Bridge J, Monga S, Baugher M. Psychometric properties of the Screen for Child Anxiety Related Emotional Disorders Scale (SCARED): a replication study. Journal of the American Academy of Child and Adolescent Psychiatry 1999;38:1230-6.

\section{Bittner 2007}

Bittner A, Egger HL, Erkanli A, Costello JE, Foley DL, Angold A. What do childhood anxiety disorders predict?. Journal of Child Psychology and Psychiatry 2007;48(12):1174-83.

\section{Bolton 2007}

Bolton P, Bass J, Betancourt T, Speelman L, Onyango G, Clougherty KF, et al. Interventions for depression symptoms among adolescent survivors of war and displacement in northern Uganda: a randomized controlled trial. JAMA 2007;298(5):519-27.

\section{Brady 1992}

Brady EU, Kendall PC. Comorbidity of anxiety and depression in children and adolescents. Psychological Bulletin 1992;111(2):244-55.

\section{Brent 1986}

Brent DA, Kalas R, Edelbrock C, Costello AJ, Dulcan MK, Conover N. Psychopathology and its relationship to suicidal ideation in childhood and adolescence. Journal of the American Academy of Child and Adolescent Psychiatry 1986;25(5):666-73.

\section{Brent 2002}

Brent DA, Birmaher B. Adolescent depression. New England Journal of Medicine 2002;347(9):667-71.

\section{Burkart 2002}

Burkart P. Children's adherence to recommended asthma selfmanagement. Pediatric Nursing 2002;28(4):409-14.

\section{Burke 1999}

Burke P, Elliott M. Depression in pediatric chronic illness. Psychosomatics 1999;40(1):5-17.

\section{Cadman 1987}

Cadman D, Boyle M, Szatmari P. Chronic illness, disability and mental and social wellbeing; finding of the Ontario child health study. Pediatrics 1987;79:805-13.

\section{Cain 2002}

Cain DJ, Seeman J. Humanistic Psychotherapies: Handbook of Research and Practice. Washington DC: American Psychological Association, 2002.

\section{Carr 2006}

Carr A. The Handbook of Child and Adolescent Clinical Psychology. 2nd Edition. London (UK): Routledge, 2006.

\section{Cates 2002}

Cates CJ. Simpson's paradox and calculation of number needed to treat from meta-analysis. BMC Medical Research Methodology 2002; $2: 1$.

\section{Christiensen 2009}

Christensen H, Griffits KM, Farrer L. Adherence in Internet interventions for anxiety and depression. Journal of Medical Internet Research 2009;11(2):e13.

\section{Cicchetti 1998}

Cicchetti D, Toth SL. The development of depression in children and adolescents. American Psychologist 1998;53(2):221-41.

\section{Compas 2012}

Compas B, Jaser SJ, Dunn ML, Rodriguez EM. Coping with chronic illness in childhood and adolescence. Annual Review of Clinical Psychology 2012;27(8):455-80.

\section{Costello 1992}

Costello EJ, Shugart MA. Above and below the threshold: severity of psychiatric symptoms and functional impairment in a pediatric sample. Pediatrics 1992;90(3):359-68.

\section{Costello 2004}

Costello EJ, Egger HL, Angold A. Developmental epidemiology of anxiety disorders. In: Ollendick TH, March JS editor(s). Phobic and Anxiety Disorders in Children and Adolescents: A Clinician's Guide to Effective Psychosocial and Pharmacological Interventions. USA: Oxford University Press, 2004:61-91.

\section{Covidence [Computer program]}

Veritas Health Innovation. Covidence. Melbourne, Australia: Veritas Health Innovation, accessed 16 August 2016.

\section{Cox 2014}

Cox GR, Callahan P, Churchill R, Hunot V, Merry SN, Parker AG, et al. Psychological therapies versus antidepressant medication, alone and in combination for depression in children and adolescents. Cochrane Database of Systematic Reviews 2014, Issue 11. [DOI: 10.1002/14651858.CD008324.pub3]

\section{Craske 2014}

Craske MG, Treanor M, Conway CC, Zbozinek T, Vervliet B. Maximizing exposure therapy: an inhibitory learning approach. Behaviour Research and Therapy 2014;58:10-23.

\section{Cuijpers 2002}

Cuijpers P, Smit F. Excess mortality in depression: a meta analysis of community studies. Journal of Affective Disorders 2002;72(3):227-36

\section{D'Zurilla 1999}

D'Zurilla TJ, Nezu AM. Problem-Solving Therapy: A Social Competence Approach to Clinical Intervention. 2nd Edition. New York: Springer, 1999.

\section{Dantzer 2003}

Dantzer C, Swendsen J, Maurice-Tison S, Salamon R. Anxiety and depression in juvenile diabetes: a critical review. Clinical Psychology Review 2003;23:787-800.

\section{Davidson 2002}

Davidson RJ, Lewis DA, Alloy LB, Amaral DG, Bush G, Cohen JD. Neural and behavioral substrates of mood and mood regulation. Biological Psychiatry 2002;52:478-502. 


\section{de Jongh 2012}

de Jongh T, Gurol-Uganci I, Vodopivek-Jamsek J, Car J, Atun R. Mobile phone messaging for facilitating self-management of long-term illnesses. Cochrane Database of Systematic Reviews 2012, Issue 12. [DOI: 10.1002/14651858.CD007459.pub2]

\section{Deeks 2000}

Deeks J. Issues in the selection for meta-analyses of binary data. 8th International Cochrane Colloquium; 2000 Oct 25-28; Cape Town (SA). The Cochrane Collaboration, 2000.

\section{Denecke 2000}

Denecke H, Kröner-Herwig B. Headache Therapy with Children and Adolescents: A Training Program (Therapeutic Practice). Göttingen: Hogrefe, 2000.

\section{Denny 2014}

Denny S, Silva M, Fleming T, Clark T, Merry S, Ameratunga S, et al. The prevalence of chronic health conditions impacting on daily functioning and the association with emotional well-being among a national sample of high school students. Journal of Adolescent Health 2014;54(4):410-5.

\section{Diamond 2002}

Diamond GS, Reis BF, Diamond GM, Siqueland L, Isaacs L. Attachment-based family therapy for depressed adolescents: a treatment development study. Journal of the American Academy of Child and Adolescent Psychiatry 2000;41(10):1190-6.

\section{Dunn 2011}

Dunn T, Casey LM, Sheffield L, Newcombe P, Chang AB. Dropout from computer-based interventions for children with chronic health conditions. Journal of Health Psychology 2011;17(3):429-42.

\section{Duval 2000}

Duval S, Tweedie R. Trim and fill: a simple funnel-plot-based method of testing and adjusting for publication bias in metaanalysis. Biometrics 2000;56(2):453-63.

\section{Eccleston 2012}

Eccleston C, Palermo TM, Fisher E, Law E. Psychological interventions for parents of children and adolescents with chronic illness. Cochrane Database of Systematic Reviews 2012, Issue 8. [DOI: 10.1002/14651858.CD009660.pub2]

\section{Eccleston 2014}

Eccleston C, Palermo TM, Williams AC, Lewandowski Holley A, Morley S, Fisher E, et al. Psychological therapies for the management of chronic and recurrent pain in children and adolescents. Cochrane Database of Systematic Reviews 2014, Issue 5. [DOI: 10.1002/14651858.CD003968.pub4]

\section{Eccleston 2015}

Eccleston C, Fisher E, Law E, Bartlett E, Palermo T. Psychological interventions for parents of children and adolescents with chronic illness. Cochrane Database of Systematic Reviews 2015, Issue 4. [DOI: 10.1002/14651858.CD009660.pub3]

\section{Eiser 1997}

Eiser C. Effects of chronic illness on children and their families. Advances in Psychiatric Treatment 1997;3:204-10.

\section{Feehan 1993}

Feehan M, McGee R, Williams SM. Mental health disorders from age 15 to age 18 years. Journal of the American Academy of Child and Adolescent Psychiatry 1993;32(6):1118-27.

\section{Feehan 1994}

Feehan M, McGee R, Raja SN, Williams SM. DSM-III-R disorders in New Zealand 18-year-olds. Australian and New Zealand Journal of Psychiatry 1994;28:87-99.

\section{Fergusson 1993}

Fergusson DM, Horwood LJ, Lynskey MT. Prevalance and comorbidity of DSM-III-R diagnoses in a birth cohort of 15 year olds. Journal of the American Academy of Child and Adolescent Psychiatry 1993;32(6):1127-35.

\section{Fergusson 2001}

Fergusson DM, Horwood LJ. The Christchurch health and development study: review of findings on child and adolescent mental health. Australian and New Zealand Journal of Psychiatry 2001;35:287-96.

\section{Fergusson 2006}

Fergusson DM, Horwood LJ, Ridder EM, Beautrais AL. Subthreshold depression in adolescence and mental health outcomes in adulthood. Archives of General Psychiatry 2005;62(1):66-72.

\section{Fielding 1999}

Fielding D, Duff A. Compliance with treatment protocols: interventions for children with chronic illness. Archives of Disease in Childhood 1999;80:196-200.

\section{Fleming 1993}

Fleming JE, Boyle MH, Offord DR. The outcome of adolescent depression in the Ontario child health study follow-up. Journal of the American Academy of Child and Adolescent Psychiatry 1993;32(1):28-33.

\section{Fleming 2015}

Fleming T, Cheek C, Merry S, Thabrew $\mathrm{H}$, Briedgeman $\mathrm{H}$, Stasiak K, et al. Serious games for the treatment or prevention of depression: a systematic review. Revista de Psicopatología y Psicología Clínica 2015;19(3):227.

\section{Fonagy 2005}

Fonagy P, Target M, Cottrell D, Phillips J, Kurtz Z. What Works for Whom: A Critical Review of Treatments for Children and Adolescents. 1st Edition. New York (NY): Guilford Press, 2005.

\section{Furakawa 2014}

Furukawa TA, Noma H, Caldwell DM, Honyashiki M, Shinohara K, Imai $\mathrm{H}$, et al. Waiting list may be a nocebo condition in psychotherapy trials: a contribution from network metaanalysis. Acta Psychiatrica Scandinavica 2014;130(3):181-92. 


\section{Gilbert 2005}

Gilbert PJ. Compassion: Conceptualisations, Research and Use in Psychotherapy. New York (NY): Brunner-Routledge, 2005.

\section{Gilbert 2009}

Gilbert PJ. The Compassionate Mind. London (UK): Constable \& Robinson, 2009.

\section{Gonzales-Tejera 2005}

González-Tejera G, Canino G, Ramírez R, Chávez L, Shrout P, Bird $\mathrm{H}$, et al. Examining minor and major depression in adolescents. Journal of Child Psychology and Psychiatry, and Allied Disciplines 2005;46(8):888-99.

\section{Goodyer 2000}

Goodyer IM, Tamplin A, Herbert J, Altham PM. Recent life events, cortisol, dehydroepiandrosterone and the onset of major depression in high-risk adolescents. British Journal of Psychiatry 2000;177(6):499-504.

\section{Gortmaker 1990}

Gortmaker SL, Walker DK, Weitzman M. Chronic conditions, socioeconomic risks and behavioural problems in children and adolescents. Pediatrics 1990;85:267-76.

\section{GRADEpro GDT [Computer program]}

McMaster University (developed by Evidence Prime). GRADEpro GDT. Hamilton (ON): McMaster University (developed by Evidence Prime), 2015.

\section{Guerney 1971}

Guerney B Jr, Stollak G, Guerney L. The practicing psychologist as educator - an alternative to the medical practitioner model. Professional Psychology 1971;2:276-82.

\section{Guyatt 1998}

Guyatt GH, Juniper EF, Walter SD, Griffith LE, Goldstein RS. Interpreting treatment effects in randomised trials. BMJ 1998;316(7132):690-3.

\section{Halfon 2010}

Halfon N, Newacheck W. Evolving notions of childhood chronic illness. JAMA 2010;303(7):665-6.

\section{Hamilton 1967}

Hamilton M. Development of a rating scale for primary depressive illness. British Journal of Social and Clinical Psychology 1967;6:278-96.

\section{Harrington 1998}

Harrington R, Clark A. Prevention and early intervention for depression in adolescence and early adult life. European Archives of Psychiatry and Clinical Neuroscience 1998;248:32-45.

\section{Hayes 1999}

Hayes SC, Strosahl K, Wilson KG. Acceptance and Commitment Therapy: An Experiential Approach to Behavior Change. New York (NY): Guilford Press, 1999.

\section{Hayes 2004}

Hayes SC, Masuda A, Bassett R, Luoma J, Guerrero LF. DBT, FAP, and ACT: how empirically oriented are the new behavior therapy technologies?. Behavior Therapy 2004;35:35-54.

\section{Hazell 2002}

Hazell P, O'Connell D, Heathcote D, Henry D. Tricyclic drugs for depression in children and adolescents. Cochrane Database of Systematic Reviews 2002, Issue 2. [DOI: 10.1002/14651858.CD002317.pub2]

\section{Hermanns 2005}

Hermanns N, Kulzer B, Krichbaum M, Kubiak T, Haak T. Affective and anxiety disorders in a German sample of diabetic patients: prevalence, comorbidity and risk factors. Diabetic Medicine 2005;22:293-300.

\section{Hetrick 2016}

Hetrick S, Cox G, Witt K, Bir J, Merry S. Cognitive behavioural therapy (CBT), third-wave CBT and interpersonal therapy (IPT) based interventions for preventing depression in children and adolescents. Cochrane Database of Systematic Reviews 2016, Issue 8. [DOI: 10.1002/14651858.CD003380.pub4]

\section{Higgins 2003}

Higgins JP, Thompson SG, Deeks JJ, Altman DG. Measuring inconsistency in meta-analyses. BMJ 2003;327(7414):557-60.

\section{Higgins 2011}

Higgins JPT, Green S, editor(s). Cochrane Handbook for Systematic Reviews of Interventions Version 5.1.0 (updated March 2011). The Cochrane Collaboration, 2011. Available from handbook.cochrane.org.

\section{Hill 2011}

Hill RM, Castellanos D, Pettit JW. Suicide-related behaviors and anxiety in children and adolescents: a review. Clinical Psychology Review 2011;31(7):1133-44.

\section{Hoffman 2008}

Hofmann SG, Asmundson GJG. Acceptance and mindfulnessbased therapy: New wave or old hat?. Clinical Psychology Review 2008;28(1):1-16.

\section{Internet World Stats 2017}

Internet World Stats. Internet usage statistics: the internet big picture. https://www.internetworldstats.com/stats.htm (accessed 20 June 2017).

\section{Jacobsen 1996}

Jacobson NS, Dobson KS, Truax PA, Addis ME, Koerner K, Gollan JK, et al. A component analysis of cognitive behavioral treatment for depression. Consulting and Clinical Psychology 1996;64(2):295-304.

\section{James 2013}

James A, James G, Cowdrey F, Soler A, Choke A. Cognitive behavioural therapy for anxiety disorders in children and adolescents. Cochrane Database of Systematic Reviews 2013, Issue 6. [DOI: 10.1002/14651858.CD004690.pub3] 


\section{Judd 2002}

Judd LL, Schettler PJ, Akiskal HS. The prevalence, clinical relevance, and public health significance of subthreshold depressions. Psychiatric Clinics of North America 2002;25(4):685-98.

\section{Kaufman1997}

Kaufman J, Birmaher B, Brent D, Rao U, Flynn C, Moreci P, et al. Schedule for Affective Disorders and Schizophrenia for SchoolAge Children - Present and Lifetime Version (KSADS-PL): initial reliability and validity data. Journal of the American Academy of Child and Adolescent Psychiatry 1997;36:980-8.

\section{Kohlenberg 1991}

Kohlenberg RJ, Tsai M. Functional Analytic Psychotherapy: Creating Intense and Curative Therapeutic Relationships. London (UK): Plenum Press, 1991.

\section{Koons 2001}

Koons CR, Robins CJ, Tweed JL, Lynch TR, Gonzalez AM, Morse JQ, et al. Efficacy of dialectical behaviour therapy in women veterans with borderline personality disorder. Behavior Therapy 2001;32(2):371-90.

\section{Kovacs 1985}

Kovacs M. The Children's Depression Inventory (CDI). Psychopharmacology Bulletin 1985;21:995-8.

\section{Kovacs 1989}

Kovacs M, Gatsonis C, Paulauskas S, Richards C. Depressive disorders in childhood. IV. A longitudinal study of comorbidity with and the risk for anxiety disorders. Archives of General Psychiatry 1989;46:776-82.

\section{Kushner 1990}

Kushner M, Sher K, Beitman B. The relation between alcohol problems and the anxiety disorders. American Journal of Psychiatry 1990;147:685-95.

\section{La Greca 1988}

La Greca AM, Dandes SK, Wick P, Shaw K, Stone WL. Development of the Social Anxiety Scale for Children: reliability and concurrent validity. Journal of Clinical Child Psychology 1988;17:84-91.

\section{Lane 2013}

Lane DA, Millane TA, Lip GYH. Psychological interventions for depression in adolescent and adult congenital heart disease. Cochrane Database of Systematic Reviews 2013, Issue 10. [DOI: 10.1002/14651858.CD004372.pub2]

\section{Lara 2000}

Lara M, Sherbourne CD, Duan N, Morales SL, Gergen P, Brook RH. An English and Spanish Pediatric Asthma Symptom Scale. Medical Care 2000;38(3):342-50.

\section{Last 1996}

Last CG, Perrin S, Hersen M, Kazdin AE. A prospective study of childhood anxiety disorders. Journal of the American Academy of Child and Adolescent Psychiatry 1996;36(11):1502-10.

\section{Last 1997}

Last CG, Hansen C, Franco N. Anxious children in adulthood: a prospective study of adjustment. Journal of the American Academy of Child and Adolescent Psychiatry 1997;36(5):645-52.

\section{Law 2011}

Law EF, Dahlquist LM, Sil S, Weiss KE, Herbert LJ, Wohlheiter K, et al. Videogame distraction using virtual reality technology for children experiencing cold pressor pain: the role of cognitive processing. Journal of Pediatric Psychology 2011;36(1):84-94.

\section{Lewinsohn 1994}

Lewinsohn PM, Roberts RE, Seeley JR, Rohde P, Gotlib IH, Hops H. Adolescent psychopathology, II: psychosocial risk factors for depression. Journal of Abnormal Psychology 1994;103(2):302-15.

\section{Lewinsohn 1998}

Lewinsohn PM, Rohde P, Seely JR. Major depressive disorder in older adolescents: prevalence, risk factors, and clinical implications. Clinical Psychology Review 1998;18(7):765-94.

\section{Lewis 2003}

Lewis M, Vitulano L. Biopsychosocial issues and risk factors in the family when the child has a chronic illness. Child and Adolescent Psychiatric Clinic of North America 2003;12:389-99.

\section{Liebman 1974}

Liebman R, Minuchin S, Baker L. An integrated treatment program for anorexia nervosa. American Journal of Psychiatry 1974;131(4):432-6.

\section{Linehan 1993}

Linehan M. Cognitive-behavioral Treatment of Borderline Personality Disorder. New York (NY): Guilford Press, 1993.

\section{Lipsitz 2013}

Lipsitz JD, Markowitz JC. Mechanisms of change in interpersonal therapy (IPT). Clinical Psychology Review 2013;33(8):1134-47.

\section{Long 2009}

Long AC, Palermo TM. Brief report: Web-based management of adolescent chronic pain: development and usability testing of an online family cognitive behavioral therapy programme. Journal of Pediatric Psychology 2009;34:511-6.

\section{Lowe 2002}

Lowe B, Breining K, Wilke S, Wellmann R, Zipfel S, Eich W. Quantitative and qualitative effects of Feldenkrais, progressive muscle relaxation, and standard medical treatment in patients after acute myocardial infarction. Psychotherapy Research 2002;12(2):179-91.

\section{Lubin 1965}

Lubin B. Adjective checklists for measurement of depression. JAMA 1965;12(1):57-62.

\section{Madanes 1981}

Madanes C. Strategic Family Therapy. San Francisco (CA): Jossey Bass, 1981. 


\section{Maier 1988}

Maier W, Buller R, Philipp M, Heuser I. The Hamilton Anxiety Scale: reliability, validity and sensitivity to change in anxiety and depressive disorders. Journal of Affective Disorders 1988;14(1):61-8.

\section{March 1997}

March JS, Parker JD, Sullivan K, Stallings P, Conners CK. The Multidimensional Anxiety Scale for Children (MASC): factor structure, reliability, and validity. Journal of the American Academy of Child and Adolescent Psychiatry 1997;36:554-65.

\section{Martell 2001}

Martell CR, Addis ME, Jacobson NS. Depression in Context: Strategies for Guided Action. New York (NY): W.W. Norton, 2001.

\section{Maslow 1970}

Maslow AH. Motivation and Personality. New York (NY): Harper and Row, 1970.

\section{McCauley 2001}

McCauley E, Pavlidis K, Kendall K. Developmental precursors of depression: the child and the social environment. The Depressed Child and Adolescent. Vol. 2, Cambridge (UK): Cambridge University Press, 2001:46-78.

\section{McQueen 2008}

McQueen D, Kennedy R, Sinason V, Maxted F. Psychoanalytic Psychotherapy After Child Abuse: The Treatment of Adults and Children who have Experienced Sexual Abuse, Violence and Neglect in Childhood. London (UK): Karnac Books Ltd, 2008.

\section{Minuchin 1978}

Minuchin S, Rosman BL, Baker L. Psychosomatic families: Anorexianervosa in context. Vol. viii, Oxford (UK): Harvard University Press, 1978.

\section{Mufson 1996}

Mufson L, Moreau D, Weissman M. Focus on relationships: interpersonal psychotherapy for adolescent depression. In: Hibbs ED, Jensen PS editor(s). Psychosocial Treatments for Child and Adolescent Disorders: Empirically Based Strategies for Clinical Practice. Washington (USA): American Psychological Association, 1996:137-56.

\section{Mufson 2004}

Mufson L, Dorta KP, Wickramaratne P, Nomura Y. A randomized effectiveness trial of interpersonal psychotherapy for depressed adolescents. Archives of General Psychiatry 2004;61(6):577-84.

\section{Myers 2002}

Myers K, Winters NC. Ten year review of rating scales. II: scales for internalizing disorders. Journal of the American Academy of Child and Adolescent Psychiatry 2002;41(6):634-59.

\section{Newacheck 1991}

Newacheck PW, McManus MA, Fox HB. Prevalence and impact of chronic Illness among adolescents. American Journal of Diseases of Childhood 1991;145:1367-73.

\section{Newton 2004}

Newton JT, Sturmey P. Development of a short form of the Treatment Evaluation Inventory for acceptability of psychological interventions. Psychological Reports 2004;94(2):475-81.

\section{NICE 2006}

National Institute for Health and Clinical Excellence (NICE). Computerised cognitive behaviour therapy for depression and anxiety. Review of Technology Appraisal 51 (TA97) 2006.

\section{Olendick 1983}

Olendick TH. Reliability and validity of the Revised Fear Survey Schedule for Children (FSSC-R). Behaviour Research and Therapy 1983;21(6):652-92.

\section{Opolski 2005}

Opolski M, Wilson I. Asthma and depression: a pragmatic review of the literature and recommendations for research. Clinical Practice and Epidemiology in Mental Health 2005;1:18.

\section{Orlowski 2015}

Orlowski SK, Lawn S, Venning A, Winsall M, Jones GM, Wyld K, et al. Participatory research as one piece of the puzzle: a systematic review of consumer involvement in design of technology-based youth mental health and wellbeing interventions. Journal of Medical Internet Research 2015;2(2):e12.

\section{Osman 2001}

Osman A, Bagge CL, Guitierrez PM, Konick LC, Cooper BA, Barrios FX. The Suicide Behaviour Questionnaire - Revised (SBQ-R). Validation with clinical and non-clinical samples. Assessment 2001;5:443-54.

\section{Ost 1987}

Ost LG. Applied relaxation: description of a coping technique and review of controlled studies. Behaviour Research and Therapy 1987;25:397-409.

\section{Pace 2011}

Pace NL. Research methods for meta-analyses. Best practice \& research. Clinical Anaesthesiology 2011;25(4):523-33.

\section{Palermo 2016b}

Palermo TM. Web-MAP 2: Internet Intervention for Adolescents With Chronic Pain. Downloaded from www.clinicaltrials.gov on 16 August 2016. NCT01316471.

\section{Pao 2011}

Pao M, Bosk A. Anxiety in medically ill children and adolescents. Depression and Anxiety 2011;28:29-39.

\section{PARS 2002}

Research Units on Pediatric Psychopharmacology Anxiety Study Group. The Pediatric Anxiety Rating Scale (PARS): development and psychometric properties. Journal of the American Academy of Child and Adolescent Psychiatry 2002;41(9):1061-9. 


\section{Paykel 1999}

Paykel ES, Scott J, Teasdale JD, Johnson AL, Garland A, Moore R, et al. Prevention of relapse in residual depression by cognitive therapy: a controlled trial. Archives of General Psychiatry 1999;56(9):829-35.

\section{Petersen 1993}

Petersen AC, Compas BE, Brooks-Gunn J, Stemmler M, Ey S, Grant KE. Depression in adolescence. American Psychologist 1993;48(2):155-68.

\section{Petersen 1995}

Petersen AC, Leffert N. Developmental issues influencing guidelines for adolescent health research: a review. Journal of Adolescent Health 1995;17:298-305.

\section{Petti 1978}

Petti TA. Depression in hospitalized child psychiatry patients: approaches to measuring depression. Journal of the American Academy of Child Psychiatry 1978;17:49-59.

\section{Pine 2009}

Pine DS, Helfinstein SM, Bar-Haim Y, Nelson E, Fox NA. Challenges in developing novel treatments for childhood disorders: lessons from research on anxiety. Neuropsychopharmacology 2009;34(1):213-28.

\section{Pinquart 2011}

Pinquart M, Shen Y. Depressive symptoms in children and adolescents with chronic physical illness: an updated metaanalysis. Journal of Pediatric Psychology 2011;36(4):375-84.

\section{Pless 1971}

Pless IB, Roghmann KJ. Chronic illness and its consequences: observations based on three epidemiologic surveys. Journal of Pediatrics 1971;79:351-59.

\section{Pop-Jordanova 2010}

Pop-Jordanova N, Gucev Z. Game-based peripheral biofeedback for stress management in children. Pediatrics International 2010;52:428-31.

\section{Poznanski 1985}

Poznanski EO, Freeman LN Mokros HB. Children's Depression Rating Scale - Revised. Psychopharmacology Bulletin 1985;21(4):979-89.

\section{Rao 1995}

Rao U, Ryan ND, Birmaher B, Dahl RE, Williamson DE, Kaufman J, et al. Unipolar depression in adolescence: clinical outcome in adulthood. Journal of the American Academy of Child and Adolescent Psychiatry 1995;43(5):566-78.

\section{Reinecke 1998}

Reinecke MA, Ryan NE, DuBios DL. Cognitive-behavioral therapy of depression and depressive symptoms during adolescence: a review and meta-analysis. Journal of the American Academy of Child and Adolescent Psychiatry 1998;37(1):26-34.

\section{RevMan 2014 [Computer program]}

Nordic Cochrane Centre, The Cochrane Collaboration. Review Manager 5 (RevMan 5). Version 5.3. Copenhagen: Nordic Cochrane Centre, The Cochrane Collaboration, 2014.

\section{Reynolds 1978}

Reynolds CR, Richmond BO. What I Think and Feel: a revised measure of children's manifest anxiety. Abnormal Child Psychology 1978;6:271-80.

\section{Rhode 1994}

Rhode P, Lewinsohn PM, Seeley JR. Are adolescents changed by an episode of major depression?. Journal of the American Academy of Child and Adolescent Psychiatry 1994;33(9):1289-98.

\section{Rhode 2013}

Rhode P, Lewinsohn PM, Klein DN, Seeley JR, Gau JM. Key characteristics of major depressive disorder occurring in childhood, adolescence, emerging adulthood, and adulthood. Clinical Psychological Science 2013;1(1):10.1177/2167702612457599. [DOI: $10.1177 / 2167702612457599]$

\section{Rogers 1951}

Rogers C. Client-centered Therapy: Its Current Practice, Implications and Theory. London (UK): Constable, 1951.

\section{Rucker 2011}

Rucker G, Schwarzer G, Carpenter JR, Binder H, Schumacher M. Treatment-effect estimates adjusted for small-study effects via a limit meta-analysis. Biostatistics 2011;12(1):122-42.

\section{Ryan 2016}

Ryan R, Hill S. How to GRADE the quality of the evidence. CCCG. http://cccrg.cochrane.org/author-resources. La Trobe University, Melbourne. Published December 1st 2016. Version 3.0. Approved (S. Hill) December 1st 2016. Accessed 1 January 2018.

\section{Sadock 2005}

Sadock BJ, Sadock VA. Kaplan \& Sadock's Comprehensive Textbook of Psychiatry. 8th Edition. Philadelphia (PA): Lippincott Williams \& Wilkins, 2005.

\section{Schneier 1992}

Schneier F, Johnson R, Hornig J, Liebowitz CD, Weissman M. Social phobia: comorbidity and morbidity in an epidemiologic sample. Archives of General Psychiatry 1992;48(2):282-8.

\section{Schulz 1995}

Schulz KF, Chalmers I, Hayes RJ, Altman DG. Empirical evidence of bias: dimensions of methodological quality associated with estimates of treatment effects in controlled trials. JAMA 1995;273:408-12.

\section{Schulz 2010}

Schulz KF, Altman DG, Moher D, CONSORT Group. CONSORT 2010 Statement: updated guidelines for reporting parallel group randomised trials. BMJ 2010;340:698-702. 


\section{Schwartz 2003}

Schwartz M, Andrasik F. Biofeedback: A Practitioner's Guide. 3rd Edition. New York (NY): Guilford Press, 2003.

\section{Seligman 1979}

Seligman MEP, Abramson LY, Semmel A, von Baeyer C. Depressive attributional style. Journal of Abnormal Psychology 1979;88:242-7.

\section{Selvini 1978}

Selvini Palazzoli M, Boscolo L, Cecchin G, Prata G. Paradox and Counterparadox. New York (NY): Aronson, 1978.

\section{Shaffer 1984}

Shaffer D, Gould MS, Brasic J, Ambrosini P, Fisher P, Bird H, et al. A Children's Global Assessment Scale (CGAS). Archives of General Psychiatry 1984;40(11):1228-31.

\section{Sharp 2014}

Sharp J, Holly D, Broomfield N. Computerized cognitive behaviour therapy for depression in people with a chronic physical illness. British Journal of Health Psychology 2014;18:729-44.

\section{Silverman 1988}

Silverman WK, Nelles WB. The Anxiety Disorders Interview Schedule for Children. Journal of the American Academy of Child and Adolescent Psychiatry 1988;27(6):772-8.

\section{Silverman 1996}

Silverman WK, Kurtines WM. Anxiety and Phobic Disorders: A Pragmatic Approach. New York (NY): Plenum Press, 1996.

\section{Siqueland 2005}

Siqueland L, Rynn M, Diamond GS. Cognitive behavioral and attachment based family therapy for anxious adolescents: phase I and II studies. Journal of Anxiety Disorders 2005;19(4):361-81.

\section{Smith 1986}

Smith NA, Seale JP, Ley P, Shaw J, Bracs PU. Effects of intervention on medication compliance in children with asthma. Medical Journal of Australia 1986;144(3):119-22.

\section{Spielberger 1973}

Spielberger CD, Edwards CD, Montuori J, Lushene R. StateTrait Anxiety Inventory for Children. Palo Alto (CA): Consulting Psychologist Press, 1973.

\section{Spielberger 1983}

Spielberger CD, Gorssuch RL, Lushene PR, Vagg PR, Jacobs GA. Manual for the State-Trait Anxiety Inventory. Palo Alto (CA): Consulting Psychologists Press, 1983.

\section{Stinson 2009}

Stinson J, Gill N, Yamada J, Holt J. A systematic review of internet-based self-management interventions for youth with health conditions. Journal of Pediatric Psychology 2009;34:495-510.

\section{Thabrew 2016}

Thabrew H, Stasiak K, Garcia-Hoyos V, Merry S. Game for health: how e-health approaches might address the psychological needs of children and young people with longterm physical conditions. Journal of Paediatrics and Child Health 2016;52(11):1012-8.

\section{Thabrew 2017a}

Thabrew H, Stasiak K, Hetrick SE, Wong S, Merry SN. Psychological therapies for anxiety and depression in children and adolescents with long-term physical conditions. Cochrane Database of Systematic Reviews 2017, Issue 1. [DOI: 10.1002/14651858.CD012488]

\section{Trautmann 2008}

Trautmann E, Kröner-Herwig B. Internet-based self-help for children and adolescents with recurrent headache: a pilot study. Behavioural and Cognitive Psychotherapy 2000;36:241-24.

\section{Uher 2012}

Uher R, Perlis RH, Placentino A, Dernovšek MZ, Henigsberg N, Mors $\mathrm{O}$, et al. Self-report and clinician-rated measures of depression severity: can one replace the other?. Depression and Anxiety 2012;29:1043-9.

\section{Van der Lee 2007}

Van der Lee JH, Mokkink LB, Grootenhuis MA, Heymans HS, Offringa M. Definitions and measurement of chronic health conditions in childhood: a systematic review. JAMA 2007;297(24):2741-51.

\section{Varni 2004}

Varni JW, Burwinkle TM, Katz ER. The PedsQL in pediatric cancer pain. A prospective longitudinal analysis of pain and emotional distress. Journal of Developmental and Behavioral Pediatrics 2004;25:239-46.

\section{Visual Rx [Computer program]}

Cates C. Visual Rx, EMB website. Dr Chris Cates, accessed 01 January 2018.

\section{Wallander 1995}

Wallander JL, Thompson RJ, Alriksson-Schmidt AR. Psychosocial adjustment of children with chronic physical conditions. In: Roberts MC editor(s). Handbook of Pediatric Psychology. 3rd Edition. New York (NY): Guilford Press, 2003:141-58.

\section{Watanabe 2007}

Watanabe N, Hunot V, Omori IM, Churchill R, Furukawa TA. Psychotherapy for depression among children and adolescents: a systematic review. Acta Psychiatrica Scandinavica 2007;116(2):84-95

\section{Weersing 2009}

Weersing VR, Rozenman M, Gonzales A. Core components of therapy in youth: do we know what to disseminate?. Behavior Modification 2009;33(1):24-27. 


\section{Weiland 1992}

Weiland SK, Pless IB, Roghmann KJ. Chronic illness and mental health problems in pediatric practice: results from a survey of primary care providers. Pediatrics 1992;89:445-9.

\section{Weisz 2006}

Weisz JA, McCarty CA, Valeri SM. Effects of psychotherapy for depression in children and adolescents: a meta-analysis. Psychological Bulletin 2006;132(1):132-149.

\section{Weisz 2017}

Weisz J, Kazdin A. Evidence-Based Psychotherapies for Children and Adolescents. 3. New York: Guildford Press, 2017.

\section{Wells 2008}

Wells A, Fisher PL. Metacognitive therapy in recurrent and persistent depression: a multiple-baseline study of a new treatment. Cognitive Therapy and Research 2008;33(3):291-300.

\section{Wells 2009}

Wells A. Metacognitive Therapy for Anxiety and Depression. New York (NY): Guilford, 2009.

\section{CHARACTERISTICS OF STUDIES}

Characteristics of included studies [ordered by study ID]

\section{Wierzbicki 1987}

Wierzbicki M, Bartlett T. The efficacy of group and individual cognitive therapy for mild depression. Cognitive Therapy and Research 1987;11(3):337-42.

\section{World Health Organization 1992}

World Health Organization. The ICD-10 Classification of Mental and Behavioral Disorders: Clinical Descriptions and Diagnostic Guidelines. Geneva: World Health Organization, 1992.

\section{References to other published versions of this review}

\section{Thabrew 2017b}

Thabrew H, Stasiak K, Hetrick SE, Wong S, Huss JH, Merry SN. eHealth interventions for anxiety and depression in children and adolescents with long-term physical conditions. Cochrane Database of Systematic Reviews 2017, Issue 1. [DOI: 10.1002/14651858.CD012489]

\section{Law 2015}

Methods Study design: randomised controlled trial

Study grouping: parallel group

\begin{tabular}{|c|c|}
\hline Participants & 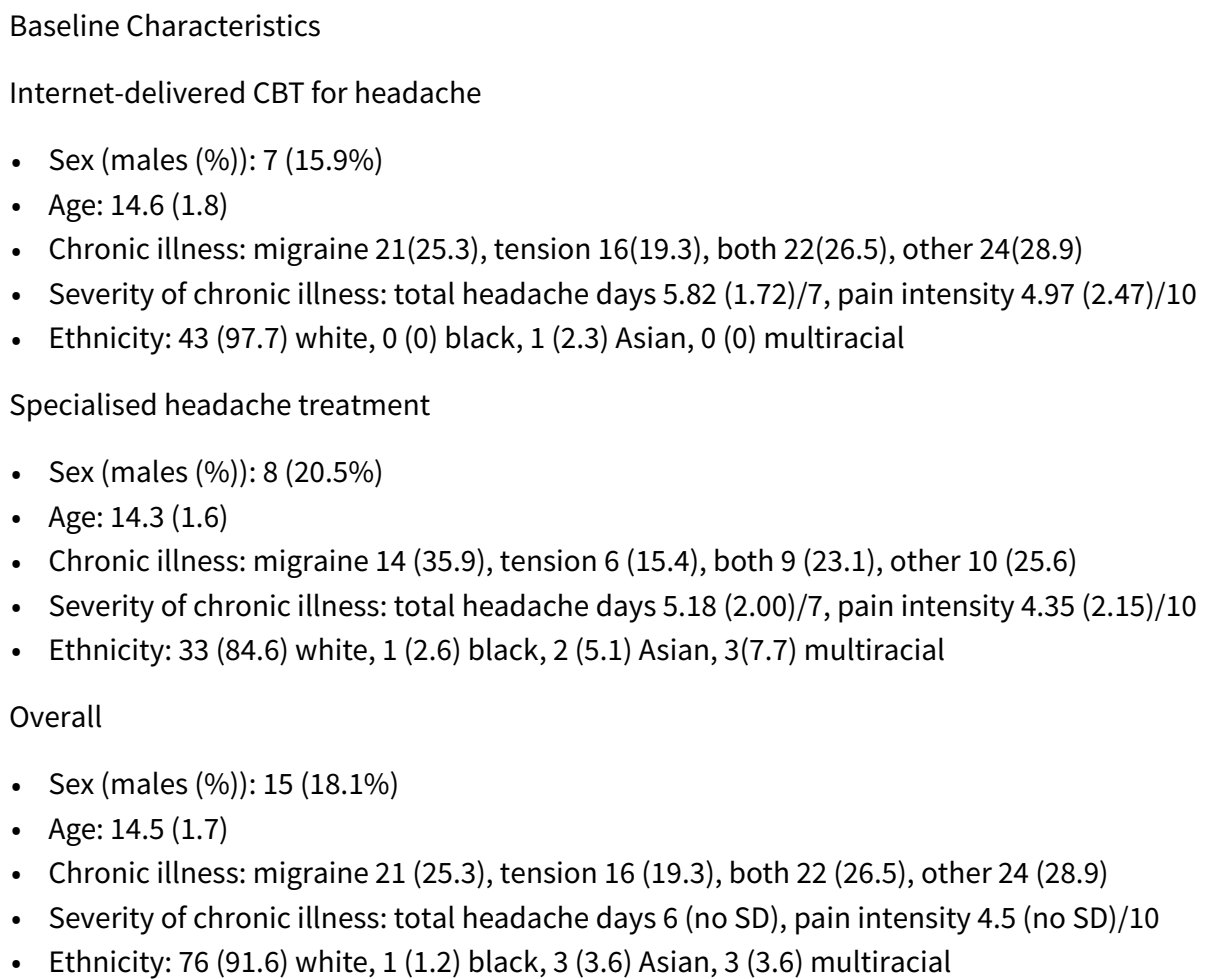 \\
\hline
\end{tabular}

E-Health interventions for anxiety and depression in children and adolescents with long-term physical conditions (Review) 
Included criteria: (1) age 11 to 17 years; (2) recurrent headache for 3 months or more as diagnosed by a paediatric neurologist; and (3) the adolescent was a new patient being evaluated in the headache clinic. "During the first clinic visit, a paediatric neurologist assigned a headache diagnosis based on the International Classification of Headache Diagnoses-II (ICHD-II). For this project, diagnoses were grouped as migraine; tension-type headache; migraine and tension-type headache; or other headache disorders." (p. 1412)

Excluded criteria: (1) the adolescent had a comorbid chronic medical condition such as diabetes, cancer, or sickle cell disease; (2) the adolescent had a developmental disability as reported by their parent; (3) the parent or adolescent was non-English speaking; or (4) the family did not have regular access to the Internet.

Pretreatment: no significant differences in demographics or baseline outcome measurements between groups.

\section{Interventions}

\section{Intervention Characteristics}

Internet-delivered CBT for Headche

- Type of e-health intervention: Web-MAP (Web-based Management of Adolescent pain) - child and parent versions

- Audience: child version and parent versions

- Number of modules: 8 child modules, 8 parent modules

- Time required and duration: 30 min per module (approximately 9 hours per family (4 hours for adolescents, 4 for parents and 1 hour online couch time))

- Description: for the Internet CBT group, the web programme was adjunctive to medical care as prescribed by the headache clinic" (p. 1412) Medication management $N=27$, psychological therapy $N=$ 10 , physical therapy $N=12$. Psychological included $C B T$ for pain management, biofeedback, or bot

Psychotherapeutic modality: CBT

- Parent or caregiver involvement: parents completed modules on pain education and goal setting, operant training, communication strategies, modelling and cognitive strategies, sleep and lifestyle interventions, and relapse prevention and maintenance.

- Parent or caregiver time involved: 30 min per module ( $4 \mathrm{~h}$ in total)

- Therapist involvement and description: review of online assignments by PhD-level psychology postdoctoral fellow and asynchronous feedback regarding review of progress, encouragement of skills practice and problem-solving barriers to implementing skills

- Therapist time involved: 5 min per assignment $=60$ min per family

- Description of modules: "Adolescents completed modules on pain education and goal setting, relaxation training, distraction strategies, cognitive strategies, sleep and lifestyle interventions, and relapse prevention and maintenance. Parents completed modules on pain education and goal setting, operant training, communication strategies, modelling and cognitive strategies, sleep and lifestyle interventions, and relapse prevention and maintenance."

- Related papers: Palermo 2009

Devices: web-based

- Based on a manual or manualised: manual developed for clinicians to guide responses

- Includes biofeedback: not applicable

- Includes homework or assignments: in six of the eight modules, parents and adolescents were given behavioral assignments focused on practice of skills taught in that module. Participants were instructed to work on the assignment for 1 week, and then to log back into the website to report on their progress with learning the skills in that module. These assignments were similar to weekly assignments used in face-to-face CBT for pain management. Assignment completion was required before participants were allowed to move on to the next module." (p. 1416)

Specialised headache treatment

- Type of e-health intervention: participants received one or more of the following interventions as recommended by their providers at the headache clinic: medication management $(N=21)$, psychological

E-Health interventions for anxiety and depression in children and adolescents with long-term physical conditions (Review) 
Law 2015 (Continued)

therapy $(N=6)$, and physical therapy $(N=11)$. Psychological therapy included face-to-face cognitive behavioral therapy for pain management, biofeedback, or both. Following completion and receipt of their 3 month follow-up assessment, families were offered the opportunity to receive Internet CBT using the same procedures below. Of the 39 families in the specialized headache treatment group, 27 (69.2\%) chose to access the Internet CBT programme after the 3 month follow-up assessment.

- Audience: child only

- Number of modules: variable number

- Time required and duration: variable over 8 weeks

- Description: "All participants continued with the medical care recommended by the headache clinic". (p. 1412)

- Psychotherapeutic modality: variable, including CBT, biofeedback for some participants

- Parent or caregiver involvement: nil

- Parent or caregiver time involved: nil

- Therapist involvement and description: as per routine care

- Therapist time involved: as per routine care

- Description of modules: N/A

- Related papers: N/A

- Devices: nil

- Based on a manual or manualised: variable

- Includes biofeedback: sSome participants only

- Includes homework or assignments: unknown
Change in severity of depression symptoms

- Outcome type: continuous outcome

- Scale: CDI

- Unit of measure: points

- Direction: lower is better

- Data value: endpoint

Change in caseness of depression

- Outcome type: dichotomous outcome

- Direction: higher is better

- Data value: endpoint

Treatment acceptability (child-related)

- Outcome type: dichotomous outcome

- Direction: higher is better

- Data value: endpoint

Improvement in QOL

- Outcome type: continuous outcome

- Direction: higher is better

- Data value: endpoint

Change in severity of anxiety

- Outcome type: continuous outcome

- Direction: lower is better

- Data value: endpoint

Change in caseness of anxiety

- Outcome type: dichotomous outcome

- Direction: lower is better

E-Health interventions for anxiety and depression in children and adolescents with long-term physical conditions (Review) 
Law 2015 (Continued)

- Data value: endpoint

Suicide-related behaviour

- Outcome type: continuous outcome

- Direction: lower is better

- Data value: endpoint

Functioning

- Outcome type: continuous outcome

- Direction: lower is better

- Data value: endpoint

- Notes: activity limitation rated on Child Activity Limitations Interview (CALI): 21 activities, 7 chosen and rated 0 to 4 (total 0 to 32)

Status of long-term physical condition (LTPC)

- Outcome type: continuous outcome

- Direction: lower is better

- Data value: endpoint

- Notes: Date for total headache days per week out of 7, pain intensity 0 to 10 scale - mean over 7 days also recorded in article

Adherence to LTPC treatment

- Outcome type: continuous outcome

- Direction: higher is better

- Data value: endpoint

School attendance

- Outcome type: continuous outcome

- Direction: higher is better

- Data value: endpoint

Economic Benefits

- Outcome type: continuous outcome

- Direction: higher is better

- Data value: endpoint

Sponsorship source: supported by Grant K24HD060068 from the National Institutes of Health/National Instituteof Child Health and Human Development (PI: Palermo)

Country: USA

Setting: multidisciplinary paediatric headache clinic at an academic health centre in north eastern United States

Comments: no comment

Authors name: Emily F. Law

Institution: Seattle Children's Research Institute

Email:emily.law@seattlechildrens.org

Address: M/S CW8-6, P.O. Box 5371, Seattle, WA98145-5005, USA 
Law 2015 (Continued)

\section{Risk of bias}

\begin{tabular}{|c|c|c|}
\hline Bias & Authors' judgement & Support for judgement \\
\hline $\begin{array}{l}\text { Random sequence genera- } \\
\text { tion (selection bias) }\end{array}$ & Low risk & $\begin{array}{l}\text { Quote: "Block randomisation with blocks of } 10 \text { was used to assign participants } \\
\text { to one of the two treatment conditions. An online number generator was used } \\
\text { to produce the blocked randomization. Participants were allocated in a } 1: 1 \text { ra- } \\
\text { tio." }\end{array}$ \\
\hline
\end{tabular}

\begin{tabular}{|c|c|c|}
\hline $\begin{array}{l}\text { Blinding of participants } \\
\text { and personnel (perfor- }\end{array}$ & High risk & $\begin{array}{l}\text { Quote: "Because of the nature of the intervention, it was not possible to blind } \\
\text { participants or research staff to group status." }\end{array}$ \\
\hline
\end{tabular}

mance bias)

All outcomes

Blinding of outcome as- Unclear risk sessment (detection bias)

Quote: "A research coordinator who was blinded to group status conducted all All outcomes assessment procedures that occurred in the clinic."

\begin{tabular}{lll}
\hline $\begin{array}{l}\text { Incomplete outcome data } \\
\text { (attrition bias) } \\
\text { All outcomes }\end{array}$ & High risk & $\begin{array}{l}\text { Judgement comment: Numbers in consort diagram do not match numbers on } \\
\text { Table 2. More than } 10 \% \text { data missing post-intervention. }\end{array}$ \\
\hline $\begin{array}{l}\text { Selective reporting (re- } \\
\text { porting bias) }\end{array}$ & Unclear risk & $\begin{array}{l}\text { Judgement comment: The trial was not pre-registered. No trial protocol avail- } \\
\text { able. }\end{array}$ \\
\hline Other bias & High risk & $\begin{array}{l}\text { Judgement comment: trial conducted by those who developed the interven- } \\
\text { tion }\end{array}$
\end{tabular}

\section{Newcombe 2012}

\begin{tabular}{ll}
\hline Methods & Study design: randomised controlled trial \\
& Study grouping: parallel group \\
\hline Participants & Baseline Characteristics \\
& Breathe Easier Online (BEO) \\
- Sex (\% male): $9 / 19(47 \%)$ & - Age: $13.41(1.99)$ \\
- Chronic illness: cystic fibrosis (CF) $\mathrm{N}=13$ (68.4\%), asthma $\mathrm{N}=5(26.3 \%)$, other $\mathrm{N}=1(5.3 \%)$ \\
- Severity of chronic illness: forced expiratory volume in 1 second $78.17(25.82)$, forced vital capacity \\
& 89.37 (26.76), forced expiratory flow mid-expiratory phase $62.71(27.28)$ \\
- Ethnicity: white $19 / 19$ (100\%) & Control intervention
\end{tabular}

E-Health interventions for anxiety and depression in children and adolescents with long-term physical conditions (Review) 
- Sex (\% male): $10.20(50 \%)$

- Age: $13.63(1.83)$

- Chronic illness: CF (9), asthma (7), other (1)

- Severity of chronic illness: forced expiratory volume in 1 second 74.19 (26.31), forced vital capacity 85.10 (26.52), forced expiratory flow mid-expiratory phase 61.32 (27.72)

- Ethnicity: white $20 / 20$ (100\%)

Overall

- Sex (\% male): unspecified

- Age: unspecified

- Chronic illness: asthma 12 (31\%), CF 22 (56\%), tracheomalacia 1 (3\%), bronchiectasis 2 (5\%)

- Severity of chronic illness: unspecified

- Ethnicity: unspecified

Included criteria: English as their primary language, had a primary diagnosis of a chronic respiratory condition, did not have any cognitive or sensory impairment that would preclude their completion of trial measures, and were deemed socially isolated or disadvantaged by hospital staff based on social indicators (single parent who is unemployed or employed part-time, and known psychological, financial difficulties (or both) from hospital social work records).

Excluded criteria: children who were unable to use a computer, had an underlying psychiatric disorder, or had a recent ( $<3$ months) hospitalisation

Pretreatment: There were no significant differences between the waiting-list control and BEO intervention groups on any of the demographic, respiratory condition, or spirometry results at baseline (time 1; all $\mathrm{F}<1$ ).

Interventions

Intervention Characteristics

Breathe Easier Online (BEO)

- Type of e-health intervention: Breathe Easier Online

- Audience: children or adolescents

- Number of modules: 5 components: My condition, My page, Daily diary, My work, and My talk

- Time required and duration: 6 modules recommended to be completed at a rate of 1 module per week over 9 weeks (but variable rate of actual completion - mentioned in discussion, but not described)

- Description: Internet-based intervention. My condition is a brief summary of each of the respiratory conditions that provided children with information about their own condition and conditions of the other children they encountered in the BEO programme. In My page, participants posted information about themselves including demographics, a photo (if they wished), favourite movie, favourite band, and a brief story about themselves. This page was visible to other BEO participants. The Daily diary section contained a checklist where participants noted the medications they had taken each day. They also recorded how often they conversed with other participants in the programme. The My talk component of the website provided opportunities for BEO participants to communicate with each other. This communication could be either asynchronous (discussion board,email) or synchronous (instant messenger). My Work section contained 6 modules that formed the focal intervention.

- Psychotherapeutic modality: Problem solving therapy based on paradigm of D'Zurilla and Nezu (D'Zurilla 1999)

- Parent or caregiver involvement: no

- Parent or caregiver time required: N/A

- Therapist involvement and description: described as 'minimal facilitator improvement'; probably involved review of homework \pm troubleshooting

- Therapist time involved: N/A

- Description of modules: the My work section of the website contained the 6 modules that formed the focal intervention

- Related papers: none

- Devices: computer (participants received a Toshiba notebook computer and a modem) 
- Based on manual or manualised: based on manual

- Includes biofeedback: no

- Includes homework assignments: yes

Control intervention

- Type of e-health intervention: waiting-list condition

- Audience: child only

- Number of modules: N/A

- Time required and duration: 9 weeks between time point 1 and time point 2

- Description: waiting-list (nothing)

- Psychotherapeutic modality: N/A

- Parent or caregiver involvement: no

- Parent or caregiver time required: N/A

- Therapist involvement and description: N/A

- Therapist time involved: N/A

- Description of modules: N/A

- Related papers: N/A

- Devices: N/A

- Based on manual or manualised: N/A

- Includes biofeedback: no

- Includes homework assignments: no

Outcomes

Change in severity of depression symptoms

- Outcome type: continuous outcome

- Reporting: partially reported

- Scale: CES-DC

- Range: 0 to 60

- Unit of measure: points

- Direction: lower is better

- Data value: endpoint

Change in caseness of depression

- Outcome type: dichotomous outcome

- Direction: lower is better

- Data value: endpoint

Treatment acceptability (child-related)

- Outcome type: continuous outcome

- Reporting: partially reported

- Scale: intervention satisfaction scale

- Range: 0 to 32

- Unit of measure: points

- Direction: higher is better

- Data value: endpoint

- Notes: narrative report on satisfaction and dropouts, but no ISS score reported despite scale being described

Improvement in QOL

- Outcome type: continuous outcome

- Direction: higher is better

- Data value: endpoint

E-Health interventions for anxiety and depression in children and adolescents with long-term physical conditions (Review) Copyright $\odot 2018$ The Cochrane Collaboration. Published by John Wiley \& Sons, Ltd. 
Change in severity of anxiety

- Outcome type: continuous outcome

- Direction: lower is better

- Data value: endpoint

Change in caseness of anxiety

- Outcome type: dichotomous outcome

- Direction: lower is better

- Data value: endpoint

Suicide-related behaviour

- Outcome type: continuous outcome

- Direction: lower is better

- Data value: endpoint

Functioning

- Outcome type: continuous outcome

- Scale: Social Problem-Solving Inventory-Revised (Short Form)

- Range: 0 to 125

- Unit of measure: points

- Direction: higher is better

- Data value: endpoint

Status of long-term physical condition

- Outcome type: continuous outcome

- Reporting: fully reported

- Scale: FEV1

- Range: 0 to 100

- Unit of measure: points

- Direction: higher is better

- Data value: endpoint

Adherence to LTPC treatment

- Outcome type: continuous outcome

- Direction: higher is better

- Data value: endpoint

School attendance

- Outcome type: continuous outcome

- Direction: higher is better

- Data value: endpoint

Economic benefits

- Outcome type: continuous outcome

- Direction: higher is better

- Data value: endpoint Foundation (Grant Number 10237). AC is funded by an NHMRC Practitioner Fellowship (Grant Number 545216).

\section{Country: Australia}

E-Health interventions for anxiety and depression in children and adolescents with long-term physical conditions (Review) 
Newcombe 2012 (Continued)

Setting: respiratory outpatient clinic

Comments: no comment

Authors name: Peter A Newcombe

Institution: University of Queensland

Email: newc@psy.uq.edu.au

Address: School of Psychology University of Queensland Bldg 24, Room s323 Brisbane, 4072 Australia

\section{Notes}

\section{Risk of bias}

\begin{tabular}{|c|c|c|}
\hline Bias & Authors' judgement & Support for judgement \\
\hline $\begin{array}{l}\text { Random sequence genera- } \\
\text { tion (selection bias) }\end{array}$ & Low risk & $\begin{array}{l}\text { Quote: "Order of random allocation was predetermined via a computer pro- } \\
\text { gram and was unknown to and concealed from the research staff." }\end{array}$ \\
\hline \multirow[t]{2}{*}{$\begin{array}{l}\text { Allocation concealment } \\
\text { (selection bias) }\end{array}$} & Unclear risk & $\begin{array}{l}\text { Quote: "Order of random allocation was predetermined via a computer pro- } \\
\text { gram and was unknown to and concealed from the research staff." }\end{array}$ \\
\hline & & Judgement comment: method of concealment not clearly described \\
\hline $\begin{array}{l}\text { Blinding of participants } \\
\text { and personnel (perfor- } \\
\text { mance bias) } \\
\text { All outcomes }\end{array}$ & High risk & $\begin{array}{l}\text { Judgement comment: not described, but as only intervention group received } \\
\text { the laptop and modem, clinical staff and patients were likely to know to which } \\
\text { group they belonged. }\end{array}$ \\
\hline $\begin{array}{l}\text { Blinding of outcome as- } \\
\text { sessment (detection bias) } \\
\text { All outcomes }\end{array}$ & Unclear risk & $\begin{array}{l}\text { Judgement comment: It is unclear whether outcome assessors were blinded. } \\
\text { Measures used to blind outcome assessors were not described. }\end{array}$ \\
\hline $\begin{array}{l}\text { Incomplete outcome data } \\
\text { (attrition bias) } \\
\text { All outcomes }\end{array}$ & Low risk & $\begin{array}{l}\text { Judgement comment: reasons for attrition and exclusions reported adequate- } \\
\text { ly. }\end{array}$ \\
\hline $\begin{array}{l}\text { Incomplete outcome data } \\
\text { (attrition bias) } \\
\text { Intervention satisfaction } \\
\text { scale }\end{array}$ & High risk & $\begin{array}{l}\text { Judgement comment: scale described, but results not provided, just descrip- } \\
\text { tion of some dimensions of treatment satisfaction. }\end{array}$ \\
\hline $\begin{array}{l}\text { Selective reporting (re- } \\
\text { porting bias) }\end{array}$ & Unclear risk & $\begin{array}{l}\text { Judgement comment: no protocol available. Results of some scales not fully } \\
\text { presented. }\end{array}$ \\
\hline Other bias & High risk & $\begin{array}{l}\text { Judgement comment: the researchers were also the developers of the BEO in- } \\
\text { tervention. }\end{array}$ \\
\hline
\end{tabular}

\section{Palermo 2009}

\begin{tabular}{ll}
\hline Methods & Study design: randomised controlled trial \\
& Study grouping: parallel group \\
\hline Participants
\end{tabular}

\section{Participants}

Baseline Characteristics 
Palermo 2009 (Continued)

Web-MAP

- Sex: male $N=6(23.1 \%)$, female $N=20(76.9 \%)$

- Age: $14.3(2.1)$

- Chronic illness: headache $\mathrm{N}=4(15.4 \%)$ a Abdominal pain $\mathrm{N}=14(53.8 \%)$, musculoskeletal pain $\mathrm{N}=8$ $(30.8 \%)$

- Severity of chronic illness: pain frequency 1 to 2 x/week $N=2$ (7.6\%), 3 to 6 x/week $N=4(15.4 \%)$, daily $\mathrm{N}=20(76.9 \%)$

- Ethnicity: white/Caucasian N = 21 (80.8\%), Hispanic $\mathrm{N}=3(11.5 \%)$, other $\mathrm{N}=2(7.7 \%)$

Waiting-list control

- Sex: male $\mathrm{N}=7(31.8 \%)$, female $\mathrm{N}=15(68.2 \%)$

- Age: $15.3(1.8)$

- Chronic illness: headache $\mathrm{N}=8(36.4 \%)$, abdominal pain $\mathrm{N}=10(45.5 \%)$, musculoskeletal pain $\mathrm{N}=4$ $(18.2 \%)$

- Severity of chronic illness: pain frequency 1 to $2 \mathrm{x} /$ week $N=4(18.2 \%), 3$ to $6 \mathrm{x} /$ week $N=3(13.6 \%)$, daily $\mathrm{N}=15(68.2 \%)$

- Ethnicity: white/Caucasian $\mathrm{N}=22(100 \%)$

Overall

- Sex: 13 males, 35 females

- Age: 11 to 17 years $(14.8$, SD 2.0$)$

- Chronic illness: chronic idiopathic pain

- Severity of chronic illness: unknown

- Ethnicity: $89.6 \%$ Caucasian

Included criteria: (a) ages 11 to 17 years, (b) chronic idiopathic pain present over the previous 3 months, (c) pain occurs at least once per week, (d) pain interferes with at least one area of daily functioning, and (e) the child was a new patient being evaluated in the specialty clinic. If families met criteria but did not have access to a computer, a laptop was provided (6\% of families).

Excluded criteria: (a) the child had a serious comorbid chronic condition (e.g. diabetes, cancer), (b) was non-English speaking, or (c) was already receiving CBT for chronic pain.

Pretreatment: no difference in gender, race, primary pain problem, or distance from treatment centre; no difference in pre-treatment measures of anxiety, pain intensity, activity limitations, depression, and parental protectiveness; slight difference in child age, with the intervention group being younger than the waiting list control group (non-significant, $P=0.07$ ).

- Type of e-health intervention: interactive website

- Audience: child and parent - separate sites

- Number of modules: $8 \times 30$ min

- Time required and duration: total treatment duration was approximately $9 \mathrm{~h}$ per family $(4 \mathrm{~h}$ child modules, $4 \mathrm{~h}$ parent modules, $1 \mathrm{~h}$ therapist time) over 8 weeks

- Description: travel-themed website with over 200 pages, including audio and video files of deep breathing and muscle relaxation instructions. At some destinations, children received online postcards from previous places they had visited, reminding them to continue to practice core treatment skills.

- Psychotherapeutic modality: CBT

- Parent or caregiver involvement: yes

- Parent or caregiver time required: 4 hours

- Therapist involvement and description: yes, PhD level psychology postgraduate fellow with experience in working with children with chronic pain; therapist manual available 
- Therapist time involved: 1 hour total (5 min per 30 min of participant involvement)

- Description of modules: The primary theoretical frameworks used to guide the intervention were cognitive behavioural and social learning frameworks. Core components of CBT were incorporated into the modules. The eight child modules were: (1) education about chronic pain, (2) recognising stress and negative emotions, (3) deep breathing and relaxation, (4) distraction, (5) cognitive skills, (6) sleep hygiene and lifestyle, (7) staying active, and (8) relapse prevention. The child modules included instruction in identifying stress, applying deep breathing and progressive muscle relaxation, and modifying cognitions about pain and functional ability. In addition, one lesson in the child programme focused on enhancing children's sleep habits (instruction in adequate sleep duration and sleep habits) and increasing their physical activity participation through goal setting and activity pacing. The eight parent modules included: (1) education about chronic pain, (2) recognizing stress and negative emotions, (3) operant strategies I, (4) operant strategies II, (5) modelling, (6) sleep hygiene and lifestyle, (7) communication, and (8) relapse prevention. Parent modules sought to provide skills in adaptive communication and interaction patterns. Operant procedures, similar to previous research were taught, including use of reinforcement for the child's maintenance of normal activity despite pain. Parents were instructed in creating privilege and point-based reward systems to target specific functional activities for reinforcement (e.g. school attendance, exercise). In addition, specialised content in the parent modules focused on the importance of modelling, supporting independence, and enhancing communication with their child.

- Related papers: Long 2009

- Devices: unknown

- Based on a manual or manualised: no

- Includes biofeedback: no

- Includes homework or assignments: yes

Waiting-list control

- Type of e-health intervention: N/A

- Audience: N/A

- Number of modules: N/A

- Time required and duration: 0 to 2 visits to specialty clinic

- Description: continuation of recommended medical care

- Psychotherapeutic modality: asked not to initiate psychotherapy during the 8 week period of the trial

- Parent or caregiver involvement: N/A

- Parent or caregiver time required: N/A

- Therapist involvement and description: N/A

- Therapist time involved: N/A

- Description of modules: N/A

- Related papers: N/A

- Devices: N/A

- Based on a manual or manualised: N/A

- Includes biofeedback: N/A

- Includes homework or assignments: N/A

\section{Outcomes}

Change in severity of depression symptoms

- Outcome type: continuous outcome

- Reporting: fully reported

- Scale: Revised Child Anxiety and Depression Scale (RCADS)

- Direction: lower is better

- Data value: endpoint

Change in caseness of depression

- Outcome type: dichotomous outcome

- Reporting: not reported

E-Health interventions for anxiety and depression in children and adolescents with long-term physical conditions (Review) 
- Outcome type: continuous outcome

- Reporting: fully reported

- Scale: Treatment Evaluation Inventory - Short Form (1 item)

- Range: strongly disagree (1) to strongly agree (5)

- Unit of measure: points

- Direction: higher is better

- Data value: endpoint

- Notes: "Children and parents in the Internet treatment group reported moderate to high ratings of treatment acceptability (child report mean $=3.55, \mathrm{SD}=0.80$, parent report mean $=3.82, \mathrm{SD}=0.50$ ). Global satisfaction was also moderate to high (child report mean $=3.68, \mathrm{SD}=0.84$, parent report mean $=4.09, \mathrm{SD}=0.61)$. Parent and child reports of acceptability and satisfaction were positively correlated $(r=0.50, P=0.02$; and $r=0.53, P=0.01$, respectively). The vast majority of children (91\%) and all parents rated the treatment as acceptable and were satisfied with the treatment (ratings > 3)." (p.211)

Improvement in QOL

- Outcome type: continuous outcome

- Reporting: not reported

Change in severity of anxiety

- Outcome type: continuous outcome

- Reporting: not reported

Change in caseness of anxiety

- Outcome type: continuous outcome

- Reporting: not reported

Suicide-related behaviour

- Outcome type: continuous outcome

- Reporting: not reported

Functioning

- Outcome type: continuous outcome

- Reporting: fully reported

- Scale: Child Activity Limitations Interview (CALI, retro)

- Range: 0 to 32

- Unit of measure: N/A

- Direction: lower is better

- Data value: endpoint

- Notes: "Activity limitations were assessed using the Child Activity Limitations Interview (CALI), which includes both retrospective and prospective versions. In the retrospective version, children respond to an item-selection list of 21 activities and choose the eight activities that are the most difficult or bothersome for them due to recurring pain. Importance of each activity to the individual child is rated on a 5-point scale from (0) not very important to (4)extremely important, to ensure that the child identifies activities that are considered relevant in his or her daily life. The primary score for the retrospective version of the CALI is derived from the difficulty ratings of the eight most difficult activities, which are obtained on a 5-point scale from (0) not very difficult to (4) extremely difficult, with total scores ranging from 0 to $32 . "$ (p. 206)

Status of long-term physical condition

- Outcome type: continuous outcome

- Reporting: fully reported

- Scale: 11-point rating scale of pain intensity retrospective

E-Health interventions for anxiety and depression in children and adolescents with long-term physical conditions (Review) Copyright $\odot 2018$ The Cochrane Collaboration. Published by John Wiley \& Sons, Ltd. 
Palermo 2009 (Continued)

- Range: 0 to 10

- Unit of measure: points

- Direction: lower is better

- Data value: endpoint

Adherence to LTPC treatment

- Outcome type: continuous outcome

- Reporting: not reported

School attendance

- Outcome type: continuous outcome

- Reporting: not reported

Economic benefits

- Outcome type: continuous outcome

- Reporting: not reported

Identification

Sponsorship source: Grant HD050674 from the National Institutes of Health/National Institute of Child Health and Human Development (PI: Palermo) and by a grant from the Doernbecher Foundation.

Country: USA

Setting: outpatient

Comments: N/A

Authors name: Tonya Palermo

Institution: Oregon Health \& Science University

Email: palermot@ohsu.edu

Address: Department of Anesthesiology and Perioperative Medicine, Oregon Health \& Science University, Portland, OR, USA

Notes

\section{Risk of bias}

Bias Authors' judgement Support for judgement

Random sequence genera- Low risk tion (selection bias)

Quote: "A fixed allocation randomisation scheme was used. Specifically, we used blocked randomisation with blocks of 10 to assign participants to the two treatment conditions during the course of randomisation. An online random number generator was used to produce the blocked randomisation."

Allocation concealment Low risk
(selection bias)

Quote: "Group assignments were identified by ID number in sealed envelopes during the 24-month recruiting period. Following completion of all pre-treatment assessments, a research co-ordinator opened the sealed envelope to reveal the group assignment. This information was then programmed into the Web-MAP system, which generated a message on the website to each trial participant revealing the instructions for the treatment phase."

$\begin{array}{lll}\begin{array}{l}\text { Blinding of participants } \\ \text { and personnel (perfor- }\end{array} & \text { High risk } & \begin{array}{l}\text { Judgement comment: waiting-list controlled trial - therefore, impossible to } \\ \text { blind participants or personnel }\end{array}\end{array}$
mance bias)

All outcomes 
Palermo 2009 (Continued)

Blinding of outcome assessment (detection bias)

All outcomes
Unclear risk Judgement comment: blinding of outcome assessors not specified

\begin{tabular}{lll}
\hline $\begin{array}{l}\text { Incomplete outcome data } \\
\text { (attrition bias) } \\
\text { All outcomes }\end{array}$ & High risk & $\begin{array}{l}\text { Judgement comment: reasons for attrition or exclusions reported adequately. } \\
\text { However, LOCF used for ITT analysis. }\end{array}$ \\
\hline $\begin{array}{l}\text { Selective reporting (re- } \\
\text { porting bias) }\end{array}$ & Unclear risk & Judgement comment: no trial protocol available \\
\hline Other bias & High risk & $\begin{array}{l}\text { Judgement comment: Authors are also the inventors of the Internet-delivered } \\
\text { family CBT programme. }\end{array}$
\end{tabular}

\section{Palermo 2016a}

Methods Study design: randomised controlled trial

Study grouping: parallel group

\section{Participants}

Baseline Characteristics

Internet-delivered CBT for adolescents

- Age: 14.63 (1.62)

- Gender (\% male): 21.7

- Ethnicity: Anglo American 92\%, Black/African American 1.4\%, Hispanic/Latino 1.4\%, other 4.5\%, missing $0.7 \%$

- Type of chronic illness: head pain $8 \%$, abdomen pain $12.3 \%$, musculoskeletal pain $37.7 \%$, multiple pain $42.0 \%$

- Severity of chronic illness: pain intensity (numerical rating scale (NRS) 0 to 10) 6.23 (1.72)

- Duration of chronic illness: not specified

Internet-delivered education

- Age: $14.70(1.72)$

- Gender (\% male): 28.1

- Ethnicity: Anglo-American 77.8\%, Black/African American 8.1\%, Hispanic/Latino 5.9\%, other 6.0\%, missing $2.2 \%$

- Type of chronic illness: head pain 5.9\%, aAbdomen pain $10.4 \%$, musculoskeletal pain $45.9 \%$, multiple pain $37.8 \%$

- Severity of chronic illness: pain intensity $5.78(1.94) / 10$

- Duration of chronic illness: not specified

Overall

- Age: $14.71(1.62)$

- Gender (\% male): 24.9

- Ethnicity: Anglo-American 85\%, Black/African American 4.8\%, Hispanic/Latino 3.7\%, other 5.0\%, missing $1.5 \%$

- Type of chronic illness: head pain 7\%, abdomen pain $11.4 \%$, musculoskeletal pain $41.8 \%$, multiple pain $39.9 \%$

- Severity of chronic illness: not available

- Duration of chronic illness: not specified 
Included criteria: (1) age 11 to 17 years, (2) chronic idiopathic pain present over the previous 3 months, (3) pain at least once per week, (4) parent report of pain interfering with at least 1 area of daily functioning, and (5) the adolescent received a new patient evaluation in one of the participating pain clinics.

Excluded criteria: (1) the adolescent had a serious comorbid psychiatric or chronic medical condition (e.g. cancer), (2) the adolescent had a developmental disability per parent report, (3) the parent or adolescent was non-English speaking, (4) the family did not have regular access to the Internet on a desktop, tablet, phone, or laptop computer, or (5) the adolescent was not residing at home (e.g. in an intensive pain rehabilitation programme)

Pretreatment: equivalent with respect to age, sex, pain condition, and parent education, pretreatment ratings of computer comfort as reported by adolescents. However, the 2 groups were significantly different on adolescent and parent race; adolescents and parents in the Internet-CBT group were more likely to be Anglo-American compared with adolescents and parents in the Internet-education group.

Interventions

\section{Intervention Characteristics}

Internet-delivered CBT for adolescents

- Type of Health intervention: Web-MAP2

- Audience: children with chronic pain and their parents

- Number of modules: 8 adolescent modules, 8 parent modules

- Time required and duration: $30 \mathrm{~min}$ each $\times 8=4$ hours; total family time estimated at 9 hrs including $1 \mathrm{hr}$ of coach time

- Description: 5 functional components of the Web-MAP2 programme, (1) treatment modules, (2) assessments and daily diaries, (3) compass (audio files of relaxation strategies), (4) passport (progress tracker), and (5) a message centre to correspond with their online coach

- Psychotherapeutic modality: CBT

- Parent or caregiver involvement: parent modules: training in operant strategies, the importance of modelling, supporting independence, and enhancing communication with their adolescent. The 8 parent modules are: (1) education about chronic pain, (2) recognizing stress and negative emotions, (3) operant strategies I (using attention and praise to increase positive coping), (4) operant strategies II (using reward to increase positive coping; strategies to support school goals), (5) modelling, (6) sleep hygiene and lifestyle, (7) communication, and (8) relapse prevention

- Parent or caregiver time required: $8 \times 30$ min modules $+1 \mathrm{hr}$ coaching time

- Therapist involvement and description: up to 5 min responses to messages from participants, up to 1 hr total time during therapy. Coaches were Masters level or PhD post-doc fellows who were trained in $\mathrm{CBT}$, undertook a standard series of training tasks, used a manual and were supervised by the author

- Therapist time involved: $1 \mathrm{hr}$ per family

- Description of modules: The programme has a travel theme; the design and treatment content of WebMAP2 was adapted from a pilot version of the programme. Cognitive behavioural, social learning, and family systems frameworks guided the interventions. There are 8 adolescent modules including: (1) education about chronic pain, (2) recognizing stress and negative emotions, (3) deep breathing and relaxation, (4) implementing coping skills at school, (5) cognitive skills (e.g. reducing negative thoughts), (6) sleep hygiene and lifestyle, (7) staying active (e.g. activity pacing, pleasant activity scheduling), and (8) relapse prevention. Vignettes, videos of peer models, illustrations, and reinforcing quizzes are used throughout the programme to increase interactivity. At some destinations, adolescents receive online postcards from previous places they have visited reminding them to practice skills. Adolescents and parents interact with the programme by identifying personal goals and entering information, which allowed tailoring and personalisation of information for weekly behavioural assignments. Adolescents and parents were asked to complete 1 module per week, designed to be analogous to weekly, clinician-delivered in-person CBT. Participants (youth and parents) spent time practicing skills and completing assignments in 6 of the 8 modules.

- Related papers: Palermo 2009

- Devices: computer

- Based on manual or manualised: yes

- Includes biofeedback: no

- Includes homework or assignments: yes 
- Type of health intervention: Internet education programme

- Audience: children with chronic pain and their parents

- Number of modules: unspecified, but adolescents and parents were instructed to log onto the web programme weekly to read the information

- Time required and duration: unspecified, but adolescents and parents were instructed to log onto the web programme weekly to read the information

- Description: 2 functional components: (1) modules with information compiled from publicly available educational web sites about paediatric chronic pain management (e.g. National Headache Foundation, etc), and (2) diary and assessments. The control web site did not provide access to behavioral and cognitive skills training.

- Psychotherapeutic modality: education about chronic pain - served as attention control condition

- Parent or caregiver involvement: yes

- Parent or caregiver time required: unspecified

- Therapist involvement and description: no

- Therapist time involved: N/A

- Description of modules: (1) modules with information compiled from publicly available educational web sites about paediatric chronic pain management (e.g. National Headache Foundation, etc), and (2) diary and assessments

- Related papers: N/A

- Devices: computer

- Based on manual or manualised: no

- Includes biofeedback: no

- Includes homework or assignments: no

Outcomes

Change in severity of depression symptoms

- Outcome type: continuous outcome

- Scale: Bath Adolescent Pain Questionnaire - depression subscale

- Range: 0 to 4

- Unit of measure: points

- Direction: lower is better

- Data value: endpoint

- Notes: "Adolescents provided difficulty ratings using a 5-point scale ( $0=$ no difficulty, $4=$ extremely difficult), with a range of 0 to 32, with higher scores indicating greater functional limitations. Average daily activity limitation scores across each assessment period were computed. Reliability and validity of the CALI has been demonstrated in school-age children and adolescents with chronic pain recruited through pain clinics and specialty clinics (e.g. rheumatology, haematology). Previous research on the prospective daily diary version of the CALI found evidence of responsiveness to changes in children's pain symptoms as evidenced by significant differences between mean CALI scores on days when pain was reported (mean $=8.2$ ) compared with pain-free days (mean $=1.7 ; \mathrm{P}=0.001)$. Mean CALI difficulty ratings also increased with increasing pain severity. Secondary outcome measures: Pain intensity: the Web-MAP online diary was used for daily assessment of presence of pain and pain intensity for 7 days at each assessment period. Pain intensity was assessed using an 11-point numerical rating scale (NRS) ( $0=$ no pain, $10=$ worst pain). The NRS has been recommended for assessment of pain intensity in adolescents with chronic pain. Average pain intensity across each assessment period was computed."

Change in caseness of depression

- Outcome type: dichotomous outcome

- Direction: lower is better

- Data value: endpoint

Treatment acceptability (child-rated)

- Outcome type: continuous outcome 
- Range: 0 to 5 scale $\times 9=$ total 9 to 45

- Unit of measure: points

- Direction: higher is better

- Data value: endpoint

- Notes:Youth acceptability reported in table. Parent acceptability also measured: adolescents and parents in the Internet education group reported moderate satisfaction and acceptability for the intervention immediately after treatment (youth mean $=29.9, S D=5.0$; parent mean $=30.2, S D=4.9$ ) and at follow-up (youth mean $=29.7, S D=5.9$; parent mean $=29.6, S D=6.0$ ). Comparatively, as hypothesized, adolescents and parents in the Internet CBT group reported significantly higher satisfaction and acceptability for the intervention immediately after treatment (youth mean $=32.2, S D=4.7, t(253) 53.84$, $\mathrm{P}=0.001$; parent mean $=33.0, \mathrm{SD}=4.5, \mathrm{t}(254) 54.89, \mathrm{P}=0.001$ ); and at follow-up (youth mean $=31.9, \mathrm{SD}$ $=4.9, \mathrm{t}(246) 53.25, \mathrm{P}=0.001$; parent mean $=32.8, \mathrm{SD}=5.2, \mathrm{t}(243) 54.48, \mathrm{P}=0.001)$. Scores indicate that both treatments are acceptable to adolescents and parents (mean $=27)$. Web programme-specific preference ratings were also in the moderate range. After treatment, adolescents assigned to the CBT condition rated significantly higher preference for the appearance of the web programme (CBT: mean $=4.1, \mathrm{SD}=0.8$ vs education: mean $=3.8, \mathrm{SD}=1.0, \mathrm{t}(252) 52.31, \mathrm{P}=0.02)$; and the travel theme (CBT: mean $=4.2, \mathrm{SD}=1.0$ vs education: mean $=3.9, \mathrm{SD}=1.1, \mathrm{t}(255) 52.60, \mathrm{P}=0.01)$; and rated the overall usefulness as higher (CBT: mean $=4.1, S D=0.8$ vs education: mean $=3.8, S D=1.0, t(253) 52.13, P=0.03$ ) compared with adolescents in the education condition. Similarly, after treatment, parents assigned to the CBT condition also rated significantly higher preference for the appearance $(C B T:$ mean $=54.4$, $\mathrm{SD}=50.7$ vs education: mean $=54.1, \mathrm{SD}=0.8, \mathrm{t}(251) 53.89, \mathrm{P}=0.0001)$; and the travel theme (CBT: mean $=4.3, \mathrm{SD}=0.9$ vs education: mean $=4.0, \mathrm{SD}=0.9, \mathrm{t}(252) 52.95, \mathrm{P}=0.003)$; and rated the overall usefulness as higher (CBT: mean $=4.5, \mathrm{SD}=0.7$ vs education: mean $=4.0, \mathrm{SD}=0.9$, t (245) 54.46, $\mathrm{P}=$ 0.0001 ) than parents in the education group. Adolescents and parents rated ease of navigation on the web programme similar for both treatment conditions.

Improvement in QOL

- Outcome type: continuous outcome

- Direction: higher is better

- Data value: endpoint

Change in severity of anxiety

- Outcome type: continuous outcome

- Scale: Bath Adolescent Pain Q'aire - pain-specific anxiety

- Range: 0 to $4 \times 7=0$ to 28 total scores

- Unit of measure: points

- Direction: lower is better

- Data value: endpoint

- Notes: "Adolescents provided difficulty ratings using a 5-point scale ( $0=$ no difficulty, $4=$ extremely difficult), with a range of 0 to 32 with higher scores indicating greater functional limitations. Average daily activity limitation scores across each assessment period were computed. Reliability and validity of the CALI has been demonstrated in school-age children and adolescents with chronic pain recruited through pain clinics and specialty clinics (e.g. rheumatology, haematology). Previous research on the prospective daily diary version of the CALI found evidence of responsiveness to changes in children's pain symptoms as evidenced by significant differences between mean CALI scores on days when pain was reported $($ mean $=8.2$ ) compared with pain-free days (mean $=1.7, P=0.001)$. Mean CALI difficulty ratings also increased with increasing pain severity. Secondary outcome measures: Pain intensity: the Web-MAP online diary was used for daily assessment of presence of pain and pain intensity for 7 days at each assessment period. Pain intensity was assessed using an 11-point numerical rating scale (NRS; $0=$ no pain, $10=$ worst pain). The NRS has been recommended for assessment of pain intensity in adolescents with chronic pain. Average pain intensity across each assessment period was computed."

Change in caseness of anxiety

- Outcome type: dichotomous outcome

- Direction: lower is better

E-Health interventions for anxiety and depression in children and adolescents with long-term physical conditions (Review) 
- Data value: endpoint

Suicide-related behaviour

- Outcome type: continuous outcome

- Direction: lower is better

- Data value: endpoint

Functioning

- Outcome type: continuous outcome

- Scale: Child Activity Limitations Interview (CALI)

- Range: 0 to $5 \times 8 / 21$ chosen activities = total 0 to 32

- Unit of measure: points

- Direction: lower is better

- Data value: endpoint

Status of long-term physical condition

- Outcome type: continuous outcome

- Scale: 11-point numerical rating scale

- Range: 0 to 10

- Unit of measure: points

- Direction: lower is better

- Data value: endpoint

Adherence to LTPC treatment

- Outcome type: continuous outcome

- Direction: higher is better

- Data value: endpoint

School attendance

- Outcome type: continuous outcome

- Direction: higher is better

- Data value: endpoint

Economic benefits

- Outcome type: continuous outcome

- Direction: higher is better

- Data value: endpoint

Sponsorship source: Eunice Kennedy Shriver National Institute of Child Health \& HumanDevelopment of the National Institutes of Health under Award Number R01HD062538 (T.M.P. [principal investigator])

Country: USA

Setting: interdisciplinary paediatric pain clinics at academic medical centres across USA and Canada

Comments: 15 centres, from which 14 sent in referrals, and patients from 12 were enrolled.

Authors name: Tonya M. Palermo

Institution: University of Washington, Seattle

Email: tonya.palermo@seattlechildrens.org

E-Health interventions for anxiety and depression in children and adolescents with long-term physical conditions (Review) 
Palermo 2016a (Continued)

Address: Department of Anesthesiology and Pain Medicine, University of Washington,Seattle, WA, USA, Center for Child Health, Behavior, and Development, SeattleChildren's Research Institute, Seattle, WA, USA

\section{Notes}

\section{Risk of bias}

\begin{tabular}{|c|c|c|}
\hline Bias & Authors' judgement & Support for judgement \\
\hline $\begin{array}{l}\text { Random sequence genera- } \\
\text { tion (selection bias) }\end{array}$ & Low risk & $\begin{array}{l}\text { Quote: "Randomization was implemented using a computer-generated ran- } \\
\text { domisation schedule to derive a randomisation assignment to } 2 \text { treatment } \\
\text { conditions in blocks of } 4 \text { for each ID number." }\end{array}$ \\
\hline \multirow[t]{2}{*}{$\begin{array}{l}\text { Allocation concealment } \\
\text { (selection bias) }\end{array}$} & Low risk & $\begin{array}{l}\text { Quote: "The randomisation assignment was programmed into the Web-MAP2 } \\
\text { system." }\end{array}$ \\
\hline & & Judgement comment: computerised allocation \\
\hline $\begin{array}{l}\text { Blinding of participants } \\
\text { and personnel (perfor- } \\
\text { mance bias) } \\
\text { All outcomes }\end{array}$ & Unclear risk & $\begin{array}{l}\text { Judgement comment: no information provided on blinding procedures or } \\
\text { whether the intended blinding was effective }\end{array}$ \\
\hline $\begin{array}{l}\text { Blinding of outcome as- } \\
\text { sessment (detection bias) } \\
\text { All outcomes }\end{array}$ & Unclear risk & $\begin{array}{l}\text { Quote: "Because all trial assessments were completed independently online, } \\
\text { there was no possible examiner bias in outcome assessments." }\end{array}$ \\
\hline $\begin{array}{l}\text { Incomplete outcome data } \\
\text { (attrition bias) } \\
\text { All outcomes }\end{array}$ & Low risk & $\begin{array}{l}\text { Judgement comment: reasons for attrition and exclusions from analysis were } \\
\text { reported }\end{array}$ \\
\hline $\begin{array}{l}\text { Selective reporting (re- } \\
\text { porting bias) }\end{array}$ & Low risk & $\begin{array}{l}\text { Quote: "The clinical trial was registered and the full protocol is avail- } \\
\text { able" (Palermo 2016b) }\end{array}$ \\
\hline Other bias & High risk & Judgement comment: trial author is inventor of Web-MAP2 \\
\hline
\end{tabular}

\section{Trautmann 2010}

\begin{tabular}{ll}
\hline Methods & Study design: randomised controlled trial \\
& Study grouping: parallel group \\
\hline Participants & Baseline Characteristics \\
& Internet-based CBT \\
- Sex (\%male): $12 / 24$ (50\%) & - Age: $13.1(2.3)$ \\
- Chronic illness: migraine 16, tension headache 7 , both 1 \\
- Duration of chronic illness: duration of headache in years (mean, SD) 3.6 (3.6) \\
- Severity of chronic illness: headache diary: frequency 11.5 (8.2), intensity 5.0 (1.8), duration 6.8 (4.0) \\
- Ethnicity: unspecified \\
Applied Relaxation \\
- Sex (\%male): $8 / 22(36 \%)$
\end{tabular}


- Age: $12.8(2.1)$

- Chronic illness: migraine 13, tension headache 3, both 6

- Duration of chronic illness: duration of headache in years (mean, SD) 2.9 (3.1)

- Severity of chronic illness: headache diary: frequency 10.3 (7.8), intensity 5.1 (1.7), duration 8.1 (6.7)

- Ethnicity: unspecified

Educational Intervention

- Sex (\% male): $10 / 19(52 \%)$

- Age: $11.9(1.6)$

- Chronic illness: migraine 4, tension headache 1 , both 0

- Duration of chronic illness: duration of headache in years (mean, SD) 2.0 (1.9)

- Severity of chronic illness: headache diary: frequency 10.7 (7.4), intensity 5.2 (1.7), duration 7.8 (5.8)

- Ethnicity: unspecified

Overall

- Sex (\% male): $30 / 66(45 \%)$

- Age: $12.7(2.2)$

- Chronic illness: recurrent headache

- Duration of chronic illness: 2.8 years $(S D=3.0)$

- Severity of chronic illness: unspecified

- Ethnicity: unspecified

Included criteria: 1 ) between the ages of 10 and 18 years, since younger children often need help from their parents. 2) suffering from primary headache (migraine, tension type headache (TTH) or combined head-ache) at least twice a month (diagnosed by their personal physician and reported by their parents), 3) be able to read and write in German, 4) have access to a personal computer and the Internet

Excluded criteria: 1) had recently started taking prophylactic medication for the headache, 2) or were in psychotherapeutic treatment

Pretreatment: no significant differences in pretreatment measures or demographic variables between 3 groups. Nor were there any between those who completed all assessments and those who dropped out part-way.

Interventions Intervention Characteristics

Internet-based CBT

- Type of e-health intervention: multimodal cognitive behavioural training (CBT)

- Audience: children and adolescents

- Number of modules: 6 modules

- Time required and duration: 0.5 to $1 \mathrm{hr} \times 6$, and email follow-up weekly over 6 weeks

- Description: "CBT was adapted from the manualised face-to-face group therapy programme devised by Denecke and Kroner-Herwig (2000) for children with recurrent headache. CBT was reduced from 8 to 6 sessions in a self-help format, and the protocol was adapted to adolescents up to 18 years. While the first module present education on headaches, the second unit focused on stress management (perception of own stress symptoms, coping with stress). In the following modules the participants acquire progressive relaxation techniques, cognitive restructuring (identification of dysfunctional cognitions regarding headache and stress and identifying functional cognitions), self-assurance strategies (being proactive and sensitive to one's own needs), as well as problem solving. Participants of the CBT were offered a CD with relaxation instructions (a full relaxation protocol involving tensing and relaxing of major muscle groups, beginning with the upper body and proceeding to the lower body), and they could download the relaxation instructions from the training website."

- Psychotherapeutic modality: cognitive behavioural therapy

- Parent or caregiver involvement: no

- Parent or caregiver time required: N/A 
- Therapist involvement and description: weekly email contact x 6 weeks +2 additional booster contacts at week 4 and 8

- Therapist time involved: 1.8 (0.55) contacts per week, average therapist time per patient $132 \mathrm{~min}(66.1)$ over treatment period

- Description of modules: Module 1: education about headaches; Module 2: stress management (perception of stress symptoms, coping with stress); Module 3 to 6: progressive relaxation techniques; cognitive restructuring (identifying dysfunctional cognitions regarding headache and stress and then identifying functional cognitions); self-assurance strategies (being pro-active and sensitive to one's own needs); problem-solving

- Related papers: related study (Trautmann 2008); CBT adapted from Denecke and Kröner-Herwig (Denecke 2000).

- Devices: computer and CD with relaxation instructions

- Based on manual or manualised: CBT was adapted from the manualised face-to-face group therapy programme devised by Denecke and Kroner-Herwig (2000) for children with recurrent headache.

- Includes biofeedback: no

- Includes homework or assignments: yes

Applied relaxation (AR)

- Type of e-health intervention: applied relaxation via CD with differential, cue-controlled and full relaxation procedures

- Audience: child only

- Number of modules: 6 sessions

- Time required and duration: the three groups underwent six weeks of the training programme with six modules weekly, including homework exercises and email contact to discuss the week's module topics with their therapists.

- Description: the self-help modules contained only several phases from the original training: progressive relaxation, cue-controlled relaxation and differential relaxation.

- Psychotherapeutic modality: relaxation

- Parent or caregiver involvement: no

- Parent or caregiver time required: N/A

- Therapist involvement and description: weekly email contact x 6 weeks +2 additional booster contacts at week 4 and 8

- Therapist time involved: 2.1 (0.42) contacts per week, average therapist time per patient $132 \mathrm{~min}(66.1)$ over treatment period

- Description of modules: 4 relaxation tracks (2 for differential relaxation, 1 for cue-controlled relaxation and 1 full relaxation protocol (same as in CBT)

- Related papers: Ost 1987

- Devices: Internet and CD with relaxation instructions

- Based on manual or manualised: AR follows the training developed by Ost (1987).

- Includes biofeedback: no

- Includes homework or assignments: yes

Educational Intervention

- Type of e-health intervention: one self-help online module and email contact with therapist

- Audience: child only

- Number of modules: 1 module

- Time required and duration: 0.5 to $1 \mathrm{hr} \times 1$ module only, then email follow-up weekly over 6 weeks

- Description: "Participants in the EDU group received only the first self-help module (education on headache), but they had the same number of email contacts as those in the CBT and AR. The emails focused on the diary records of the previous week (e.g. Did you have any headache last week? What did you do?), rather than on cognitive behavioral elements or applied relaxation instructions. This condition served as an active control group."

- Psychotherapeutic modality: education about headache, which served as an active control

- Parent or caregiver involvement: no 
- Parent or caregiver time required: N/A

- Therapist involvement and description: weekly email contact x 6 weeks +2 additional booster contacts at week 4 and 8

- Therapist time involved: 2.2 (0.57) contacts per week, average therapist time per patient $132 \mathrm{~min}(66.1)$ over treatment period

- Description of modules: the emails focused on the diary records of the previous week (e.g. Did you have any headache last week? What did you do?), rather than on cognitive-behavioral elements or applied relaxation instructions.

- Related papers: N/A

- Devices: Internet (1 session) and pain diary

- Based on manual or manualised: no

- Includes biofeedback: no

- Includes homework or assignments: pain diary

Outcomes

Change in severity of depression symptoms

- Outcome type: continuous outcome

- Direction: lower is better

- Data value: endpoint

Change in caseness of depression

- Outcome type: dichotomous outcome

- Direction: lower is better

- Data value: endpoint

Treatment acceptability (child-rated)

- Outcome type: continuous outcome

- Scale: Patient Therapist Alliance scale

- Range: 0 to 3

- Unit of measure: points

- Direction: higher is better

- Data value: endpoint

- Notes: 2 subscales only: patient or therapist assistance and helping to cope with problems (rated 0 to 3 , higher $=$ better)

Improvement in QOL

- Outcome type: continuous outcome

- Scale: KINDL-R

- Unit of measure: points

- Direction: higher is better

- Data value: endpoint

- Notes: 6 dimensions of HR-QOL (physical, general, family functioning, self-esteem, social functioning). 24 items

Change in severity of anxiety

- Outcome type: continuous outcome

- Direction: lower is better

- Data value: endpoint

Change in caseness of anxiety

- Outcome type: dichotomous outcome

- Direction: lower is better

- Data value: endpoint

E-Health interventions for anxiety and depression in children and adolescents with long-term physical conditions (Review) Copyright $\odot 2018$ The Cochrane Collaboration. Published by John Wiley \& Sons, Ltd. 
Trautmann 2010 (Continued)

Suicide-related behaviour

- Outcome type: continuous outcome

- Direction: lower is better

- Data value: endpoint

Functioning

- Outcome type: continuous outcome

- Direction: higher is better

- Data value: endpoint

Status of long-term physical condition

- Outcome type: continuous outcome

- Scale: headache diary - frequency of headache

- Unit of measure: number of headaches per week

- Direction: lower is better

- Data value: endpoint

Adherence to LTPC treatment

- Outcome type: continuous outcome

- Direction: higher is better

- Data value: endpoint

School attendance

- Outcome type: continuous outcome

- Direction: higher is better

- Data value: endpoint

Economic benefits

- Outcome type: continuous outcome

- Direction: higher is better

- Data value: endpoint

Identification

Sponsorship source: German Research Foundation (Number: KR756/16-2)

Country: Germany

Setting: community sample recruited through advertisements

Comments: no comment

Authors name: Ellen Trautmann

Institution: Department of Clinical Psychology and Psychotherapy, University of Gottingen, Germany

Email: ekrembe@uni-goettingen.de

Address: Department of Clinical Psychology and Psychotherapy, University of Gottingen, GoBlerstr.

14,37073, Gottingen, Germany

Notes

\section{Risk of bias}


Trautmann 2010 (Continued)

Random sequence genera- Low risk Quote: "The randomly ordered list of groups was used to assign sequentialtion (selection bias)

ly enrolled participants to two intervention groups ( $N 1 / 424, N 1 / 422$ ), and the active control condition ( $N 1 / 419$ ). The first author randomly selected participants according to a computer- generated randomisation list by using the 'select cases' random selection option in the statistical software program SPSS 15.0."

Allocation concealment Unclear risk Judgement comment: not stated

(selection bias)

Blinding of participants Unclear risk Judgement comment: not stated
and personnel (performance bias)

All outcomes

\begin{tabular}{lll}
\hline $\begin{array}{l}\text { Blinding of outcome as- } \\
\text { sessment (detection bias) } \\
\text { All outcomes }\end{array}$ & Unclear risk & Judgement comment: not stated \\
\hline $\begin{array}{l}\text { Incomplete outcome data } \\
\text { (attrition bias) } \\
\text { All outcomes }\end{array}$ & High risk & $\begin{array}{l}\text { Judgement comment: reasons for attrition or exclusions reported adequately. } \\
\text { More than } 10 \% \text { data missing postintervention. }\end{array}$ \\
\hline $\begin{array}{l}\text { Selective reporting (re- } \\
\text { porting bias) }\end{array}$ & Unclear risk & Judgement comment: protocol unavailable \\
\hline Other bias & High risk & $\begin{array}{l}\text { Judgement comment: trial authors were also inventors of the internet-based } \\
\text { CBT programme. }\end{array}$ \\
\hline
\end{tabular}

CBT = Cognitive Behavioural Therapy

$\mathrm{CDI}=$ Child Drepression inventory

$\mathrm{QOL}=$ Quality of Life

$\mathrm{SD}=$ Standard Deviation

$M=$ Mean

$\mathrm{CD}=$ Compact Disc

Characteristics of excluded studies [ordered by study ID]

\begin{tabular}{ll}
\hline Study & Reason for exclusion \\
\hline Al-Haggar 2006 & Neither changes in anxiety nor in depression were measured during this trial \\
\hline Alemi 2014 & Not e-health intervention \\
\hline Berndt 2014 & Neither changes in anxiety nor in depression were measured during this trial \\
\hline Blocher 2013 & Wrong trial design \\
\hline Fernandes 2015 & Changes in neither anxiety nor depression were measured during this trial \\
\hline Hanberger 2013 & Changes in neither anxiety nor depression were measured during this trial \\
\hline Holden 1999 & Wrong trial design \\
\hline Holden 2002 & Wrong trial design \\
\hline
\end{tabular}

E-Health interventions for anxiety and depression in children and adolescents with long-term physical conditions (Review) Copyright ๑ 2018 The Cochrane Collaboration. Published by John Wiley \& Sons, Ltd. 


\begin{tabular}{|c|c|}
\hline Study & Reason for exclusion \\
\hline Ketchen 2006 & Wrong trial design \\
\hline Kotses 1991 & No e-health intervention included \\
\hline Li 2011 & Wrong trial design \\
\hline Liu 2001 & No e-health intervention included \\
\hline Miller 2012 & Not an individual trial report \\
\hline Newton 2013 & Changes in neither anxiety nor depression were measured during this trial \\
\hline Nijhof 2011 & Changes in neither anxiety nor depression were measured during this trial \\
\hline O'Hea 2013 & Wrong trial population \\
\hline Pham 2016 & Wrong trial population \\
\hline Piaserico 2016 & No e-health intervention included \\
\hline Reigada 2013 & Wrong trial design \\
\hline Sansom-Daly 2012 & No e-health intervention included \\
\hline Seitz 2014 & Wrong trial design \\
\hline Stinson 2010 & Neither changes in anxiety nor in depression were measured during this trial \\
\hline Tung 2015 & Wrong trial design \\
\hline Yetwin 2012 & No e-health intervention included \\
\hline Zinchenko 2014 & No e-health intervention included \\
\hline
\end{tabular}

Characteristics of studies awaiting assessment [ordered by study ID]

Aubin 2014

\begin{tabular}{ll}
\hline Methods & Randomised controlled trial \\
\hline Participants & $\begin{array}{l}\text { Young adults } 18 \text { to } 35 \text { years in the USA. Proportions of different ages not stated in conference ab- } \\
\text { stract. }\end{array}$ \\
\hline Interventions & $\begin{array}{l}\text { Three thematic sessions via Skype or face to face: Boxing the Cancer, Hey it's still the same me; Sex, } \\
\text { fertility, relationships and everything in between (no name provided for intervention) vs unclear } \\
\text { control intervention }\end{array}$ \\
\hline
\end{tabular}

Outcomes

Significant difference in self-efficacy reported between pre- and postintervention, but not between postintervention and follow-up, using a self-report questionnaire. No changes in mood reported between pre- and postintervention using an unnamed scale. Actual data not provided in conference abstract

Notes

Despite the likelihood this was a trial of adults, it was rated 'awaiting classification' as confirmation could not be obtained from the authors 
Blackwell 2012

\begin{tabular}{ll}
\hline Methods & Randomised controlled trial \\
\hline Participants & $\begin{array}{l}\mathrm{N}=94 \text { adolescents with cystic fibrosis (mean age } 15.69 \text { years, } 62 \% \text { female; } 57 \% \text { Caucasian, } 16 \% \text { His }- \\
\text { panic, } 9 \% \text { African American; mildly reduced lung function (predicted FEV } 1=79.89 \%, S D=25.90) \text { in } \\
\text { the USA }\end{array}$
\end{tabular}

$\begin{array}{ll}\text { Interventions } & \text { CFfone (online peer support programme for adolescents and young adults with cystic fibrosis) vs } \\ \text { CF educational website }\end{array}$

\begin{tabular}{ll}
\hline Outcomes & Preliminary results (baseline data) presented in conference abstract. Participants who felt more \\
& supported by friends had lower depressive symptoms $(r=-0.26, P<0.01)$, and anxiety symptoms $(r$ \\
& $=0.19, P=0.04)$, measured using the Hospital Anxiety and Depression Scale (HADS) at baseline, 3 \\
months, 6 months, and 9 months
\end{tabular}

\begin{tabular}{ll}
\hline Notes & $\begin{array}{l}\text { Full paper and final results of changes in anxiety and depression over time not available despite } \\
\text { contacting the authors }\end{array}$ \\
\hline
\end{tabular}

Quittner 2013

\begin{tabular}{ll}
\hline Methods & Randomised controlled trial \\
\hline Participants & $\begin{array}{l}\mathrm{N}=88 \text { adolescents and young adults with cystic fibrosis (mean age } 15.95 \text { years, } 63 \% \text { female, FEV1 }= \\
80.28 \%) \text { in the USA }\end{array}$ \\
\hline Interventions & $\begin{array}{l}\text { CFfone (online peer support programme for adolescents and young adults with cystic fibrosis) vs } \\
\text { educational website }\end{array}$ \\
\hline Outcomes & $\begin{array}{l}\text { Preliminary assessment revealed that participants in the experimental group reported fewer de- } \\
\text { pressive symptoms ( }(86)=4.19, \mathrm{P}<0.05) \text { measured using the Hospital Anxiety and Depression } \\
\text { Scale (HADS) }\end{array}$ \\
\hline Notes & $\begin{array}{l}\text { Trial paused due to funding issues, according to lead author. Final results not available and raw da- } \\
\text { ta not forwarded for independent analysis despite request }\end{array}$ \\
\hline
\end{tabular}

Sansom-Daly 2014

\begin{tabular}{ll}
\hline Methods & Randomised controlled trial \\
\hline Participants & $\mathrm{N}=21$ adolescents and young adults (aged 15 to 25 years) in Australia with cancer \\
\hline Interventions & $\begin{array}{l}\text { ReCaPTure LiFe (Resilience and Coping skills for young People To Live well Following Cancer) vs on- } \\
\text { line peer support group control intervention }\end{array}$ \\
\hline Outcomes & $\begin{array}{l}\text { Feasibiliy, acceptability, and preliminary efficacy of ReCaPTure examined. 51\% response rate ob- } \\
\text { tained across five states in the country. Preliminary data indicated an improvement in anxiety (P= } \\
0.015) \text { using an unnamed scale and at an unstated timeframe }\end{array}$ \\
\hline Notes & $\begin{array}{l}\text { Limited amount of data presented in conference abstract. Further details not available despite } \\
\text { contacting authors. }\end{array}$ \\
\hline
\end{tabular}


Sansom-Daly 2015

\begin{tabular}{ll}
\hline Methods & Case series of a subset of participants undertaking a randomised controlled trial \\
\hline Participants & $\mathrm{N}=35$ adolescents and young adults (aged 15 to 25 years) in Australia with cancer \\
\hline Interventions & $\begin{array}{l}\text { ReCaPTure LiFe (Resilience and Coping skills for young People To Live well Following Cancer) vs on- } \\
\text { line peer support group control intervention }\end{array}$ \\
\hline Outcomes & $\begin{array}{l}\text { Ethical and clinical issues associated with e-health intervention use examined in a subset of partici- } \\
\text { pants during a randomised controlled trial using unnamed scales at an unstated timeframe }\end{array}$ \\
\hline Notes & $\begin{array}{l}\text { Limited amount of data presented in conference abstract. Further details not available despite } \\
\text { contacting authors. }\end{array}$ \\
\hline
\end{tabular}

$\mathrm{N}=$ number

Characteristics of ongoing studies [ordered by study ID]

Benson 2015

Trial name or title Web-based CBT for symptoms of mild-to-moderate anxiety and depression in youth with chronic illness

\begin{tabular}{ll}
\hline Methods & Open label single-group assignment \\
\hline Participants & $\begin{array}{l}\text { Young people aged between } 15 \text { and } 22 \text { years with inflammatory bowel disease, systemic lupus ery- } \\
\text { thematosus, juvenile idiopathic arthritis, anxiety, and depression }\end{array}$ \\
\hline Interventions & Web-based CBT (no control group) \\
\hline Outcomes & $\begin{array}{l}\text { Primary outcome: programme completion rates. Secondary outcomes: change in anxiety symp- } \\
\text { toms (measured using Generalised Anxiety Disorder - 7-item (GAD-7) scales), change in depression } \\
\text { symptoms (measured using Patient Health Questionnaire (PHQ-9) scales), change in patient acti- } \\
\text { vation measure, change in quality of life (measured using the Pediatric Quality of Life Scale (Peds- } \\
\text { QL)). All scales will be completed at baseline and at } 3 \text { months }\end{array}$ \\
\hline Starting date & January 2014 \\
\hline Contact information & Rachel Bensen, Stanford University, USA \\
\hline
\end{tabular}

Notes

Cheng 2013

\begin{tabular}{ll}
\hline Trial name or title & None provided \\
\hline Methods & Randomised controlled trial \\
\hline Participants & $\begin{array}{l}\mathrm{N}=60 \text { adolescents with sickle cell disease aged } 12 \text { to } 18 \text { years recruited from the Children's Health- } \\
\text { care of Atlanta sickle cell clinic }\end{array}$ \\
\hline Interventions & $\begin{array}{l}\text { iACT, an interactive mHealth monitoring system to enhance psychotherapy vs standard Acceptance } \\
\text { and Committment Therapy (ACT) over } 6 \text { months }\end{array}$ \\
\hline
\end{tabular}


Cheng 2013 (Continued)

\begin{tabular}{ll} 
Outcomes & None stated \\
\hline Starting date & Not stated \\
\hline Contact information & $\begin{array}{l}\text { Chihwen Cheng, Department of Georgia Research Alliance, Hewlett Packard (HP) an Microsoft Re- } \\
\text { seach. Email:cwcheng83@gatech.edu }\end{array}$ \\
\hline Notes & Partly research grant-funded, partly industry-funded trial \\
\hline
\end{tabular}

\section{Clarke 2015}

\begin{tabular}{ll}
\hline Trial name or title & None stated \\
\hline Methods & Randomised controlled trial \\
\hline Participants & $\begin{array}{l}\mathrm{N}=280 \text { young people with type } 1 \text { diabetes will be recruited from diabetic services at three hospi- } \\
\text { tals in Sydney: Sydney Children's Hospital, Westmead Hospital, and St Vincent's Hospital }\end{array}$ \\
\hline Interventions & $\begin{array}{l}\text { MyCompass (mobile phone and web-based intervention for improving mental well-being in young } \\
\text { people with type 1 diabetes) vs active placebo control intervention over } 8 \text { weeks }\end{array}$ \\
\hline Outcomes & $\begin{array}{l}\text { Multiple outcomes including Patient Health Questionnaire-9 (PHQ-9) and the Generalized Anxiety } \\
\text { Disorder - } 7 \text { item (GAD-7) scales will be completed at baseline, post-intervention, and 3 month fol- } \\
\text { low-up }\end{array}$ \\
\hline Starting date & Not stated \\
\hline Contact information & $\begin{array}{l}\text { Janine Clarke, Black Dog Institute and UNSW, Hospital Road, Prince of Wales Hospital, Sydney } \\
\text { 2031, Australia. Email: janine.clarke@unsw.edu.au }\end{array}$ \\
\hline Notes &
\end{tabular}

\section{Kaufman 2017}

\begin{tabular}{ll}
\hline Trial name or title & In-Person vs e-health mindfulness-based intervention for adolescents with chronic illness \\
\hline Methods & Randomised controlled trial \\
\hline Participants & $\begin{array}{l}60 \text { adolescents aged } 13 \text { to } 18 \text { years with chronic illness attending the Hospital for Sick Kids, Toron- } \\
\text { to, Canada }\end{array}$ \\
\hline Interventions & MARS-A e-health mindfulness-based intervention vs face-to-face mindfulness intervention \\
\hline Outcomes & $\begin{array}{l}\text { Primary outcome: mindfulness skill acquisition. Secondary outcomes: change in anxiety and de- } \\
\text { pression scores (measured via DASS-21 questionnaire), appreciation of mindfulness intervention, } \\
\text { perception of illness, salivary cortisol levels, self-esteem. All outcomes will be collected at baseline, } \\
\text { and } 8 \text { weekly over } 6 \text { months. }\end{array}$ \\
\hline Starting date & Not stated \\
\hline Contact information & Miriam Kaufman, Hospital for Sick Kids, Toronto, Canada \\
\hline
\end{tabular}


Kaufman 2017 (Continued)

Notes

Mattson 2013

\begin{tabular}{ll}
\hline Trial name or title & None stated \\
\hline Methods & Randomised controlled trial \\
\hline Participants & $\begin{array}{l}1300 \text { adolescents and adults with cancer will be recruited from three hospitals in Sweden (as long } \\
\text { as they can provide consent, understand the written material, and are not in need of constant care) }\end{array}$ \\
\hline Interventions & $\begin{array}{l}\text { U }=\text { Care (internet-based stepped care with interactive support and cognitive behaviour therapy) vs } \\
\text { standard care }\end{array}$ \\
\hline Outcomes & $\begin{array}{l}\text { Multiple outcomes including the Hospital Anxiety and Depression Scale (HADS) and Mont- } \\
\text { gomery-Asberg Depression Rating Scale (MADRS) will be completed at baseline and 1, 4, 7, 10, 18, } \\
\text { and 24 months after inclusion }\end{array}$ \\
\hline Starting date & $\begin{array}{l}\text { March 2013 (duration 18 months) } \\
\text { Contact information }\end{array}$ \\
\hline
\end{tabular}

Notes

$\mathrm{CBT}=$ cognitive behavioural therapy

$\mathrm{N}=$ number

DATA AND ANALYSES

Comparison 1. E-health interventions vs any comparator

\begin{tabular}{|c|c|c|c|c|}
\hline Outcome or subgroup title & No. of studies & $\begin{array}{l}\text { No. of partici- } \\
\text { pants }\end{array}$ & Statistical method & Effect size \\
\hline 1 Depression postintervention & 5 & 441 & $\begin{array}{l}\text { Std. Mean Difference (IV, Random, } \\
95 \% \mathrm{CI})\end{array}$ & $-0.06[-0.35,0.23]$ \\
\hline $\begin{array}{l}2 \text { Depression 3- to 6-month fol- } \\
\text { low-up }\end{array}$ & 3 & 339 & $\begin{array}{l}\text { Std. Mean Difference (IV, Random, } \\
95 \% \mathrm{CI} \text { ) }\end{array}$ & $0.04[-0.18,0.25]$ \\
\hline 3 Anxiety postintervention & 2 & 324 & $\begin{array}{l}\text { Std. Mean Difference (IV, Random, } \\
95 \% \mathrm{CI} \text { ) }\end{array}$ & $-0.07[-0.29,0.14]$ \\
\hline 4 Anxiety 3- to 6-month follow-up & 2 & 319 & $\begin{array}{l}\text { Std. Mean Difference (IV, Random, } \\
95 \% \mathrm{CI} \text { ) }\end{array}$ & $0.02[-0.20,0.24]$ \\
\hline $\begin{array}{l}5 \text { Treatment acceptability postin- } \\
\text { tervention }\end{array}$ & 2 & 304 & $\begin{array}{l}\text { Std. Mean Difference (IV, Random, } \\
95 \% \mathrm{CI} \text { ) }\end{array}$ & $0.46[0.23,0.69]$ \\
\hline 6 Quality of life postintervention & 1 & 34 & $\begin{array}{l}\text { Mean Difference (IV, Random, } \\
95 \% \mathrm{Cl} \text { ) }\end{array}$ & $-0.30[-0.54,-0.06]$ \\
\hline
\end{tabular}




\begin{tabular}{|c|c|c|c|c|}
\hline Outcome or subgroup title & No. of studies & $\begin{array}{l}\text { No. of partici- } \\
\text { pants }\end{array}$ & Statistical method & Effect size \\
\hline $\begin{array}{l}7 \text { Quality of life 3- to 6-month fol- } \\
\text { low-up }\end{array}$ & 1 & 22 & $\begin{array}{l}\text { Mean Difference (IV, Random, } \\
95 \% \mathrm{CI} \text { ) }\end{array}$ & $0.10[-0.19,0.39]$ \\
\hline 8 Functioning postintervention & 3 & 368 & $\begin{array}{l}\text { Std. Mean Difference (IV, Random, } \\
95 \% \mathrm{CI} \text { ) }\end{array}$ & $-0.08[-0.33,0.18]$ \\
\hline $\begin{array}{l}9 \text { Functioning 3- to 6-month fol- } \\
\text { low-up }\end{array}$ & 2 & 319 & $\begin{array}{l}\text { Std. Mean Difference (IV, Random, } \\
95 \% \mathrm{CI})\end{array}$ & $-0.13[-0.35,0.09]$ \\
\hline $\begin{array}{l}10 \text { Status of long-term physical } \\
\text { condition postintervention }\end{array}$ & 5 & 463 & $\begin{array}{l}\text { Std. Mean Difference (IV, Random, } \\
95 \% \mathrm{CI} \text { ) }\end{array}$ & $0.06[-0.12,0.24]$ \\
\hline $\begin{array}{l}11 \text { Status of long-term physi- } \\
\text { cal condition 3- to 6-month fol- } \\
\text { low-up }\end{array}$ & 3 & 340 & $\begin{array}{l}\text { Std. Mean Difference (IV, Random, } \\
95 \% \mathrm{CI} \text { ) }\end{array}$ & $0.10[-0.11,0.32]$ \\
\hline
\end{tabular}

Analysis 1.1. Comparison $1 \mathrm{E}$-health interventions vs any comparator, Outcome 1 Depression postintervention.

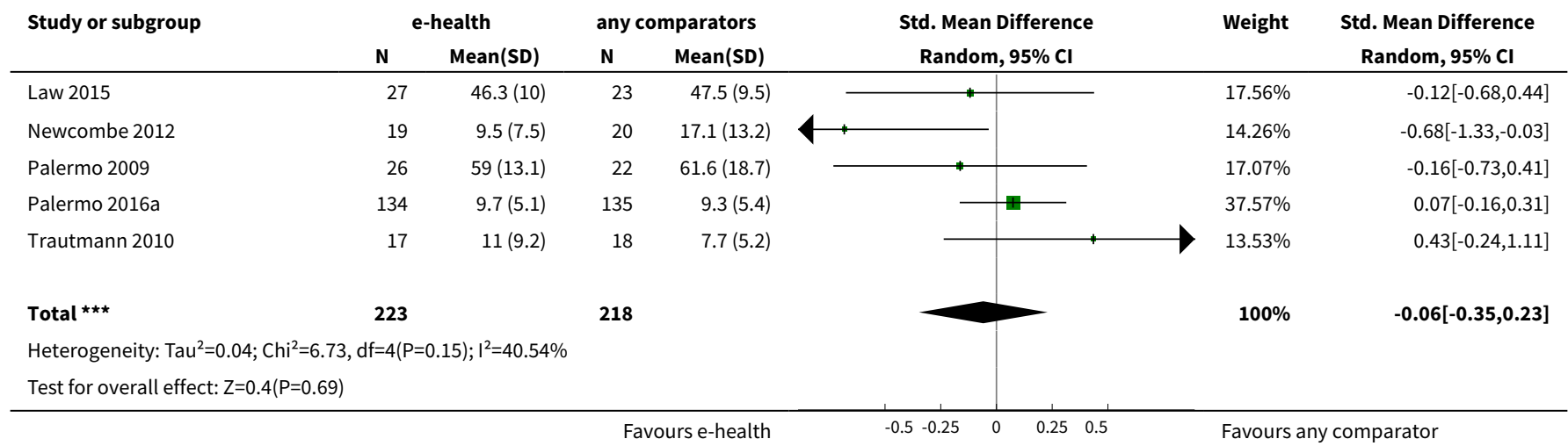

Analysis 1.2. Comparison 1 E-health interventions vs any comparator, Outcome 2 Depression 3- to 6-month follow-up.

\begin{tabular}{|c|c|c|c|c|c|c|c|}
\hline \multirow{3}{*}{$\begin{array}{l}\text { Study or subgroup } \\
\text { Law } 2015\end{array}$} & \multicolumn{2}{|c|}{ e-health } & \multicolumn{2}{|c|}{ any comparators } & \multirow{2}{*}{$\begin{array}{c}\text { Std. Mean Difference } \\
\text { Random, } 95 \% \mathrm{Cl}\end{array}$} & \multirow[t]{2}{*}{ Weight } & \multirow{2}{*}{$\begin{array}{c}\text { Std. Mean Difference } \\
\text { Random, } 95 \% \mathrm{CI}\end{array}$} \\
\hline & $\mathbf{N}$ & Mean(SD) & $\mathbf{N}$ & Mean(SD) & & & \\
\hline & 28 & $44.8(9.5)$ & 23 & $43.7(6.5)$ & & $14.9 \%$ & $0.12[-0.43,0.67]$ \\
\hline Palermo 2016a & 134 & $9.6(5.1)$ & 135 & $9.5(5.6)$ & & $79.52 \%$ & $0.01[-0.23,0.25]$ \\
\hline Trautmann 2010 & 10 & $7.7(7.1)$ & 9 & $6.6(3.7)$ & & $5.57 \%$ & $0.18[-0.72,1.09]$ \\
\hline Total $\star \star \star ~$ & 172 & & 167 & & & $100 \%$ & $0.04[-0.18,0.25]$ \\
\hline \multicolumn{8}{|c|}{ Test for overall effect: $Z=0.34(P=0.73)$} \\
\hline
\end{tabular}


Analysis 1.3. Comparison $1 \mathrm{E}$-health interventions vs any comparator, Outcome 3 Anxiety postintervention.

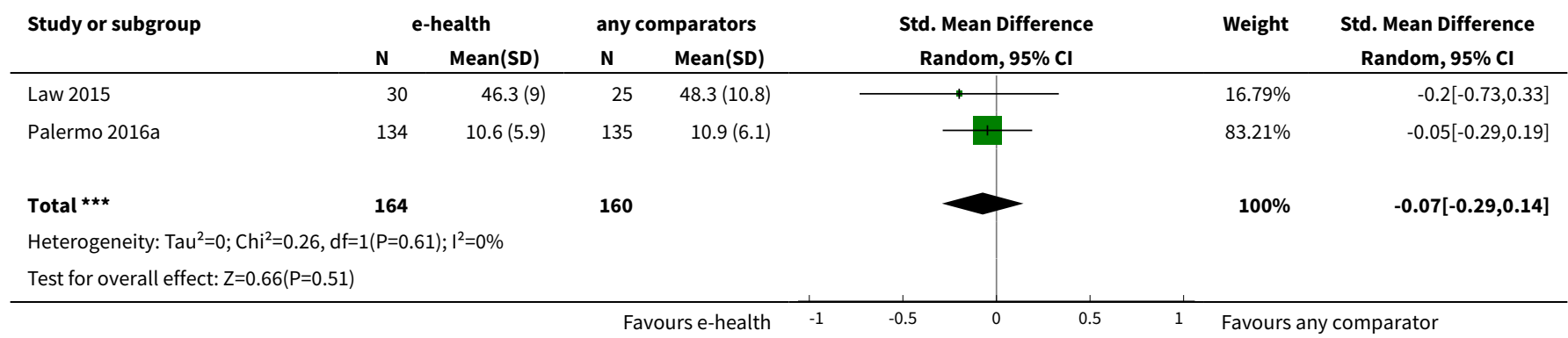

Analysis 1.4. Comparison 1 E-health interventions vs any comparator, Outcome 4 Anxiety 3- to 6-month follow-up.

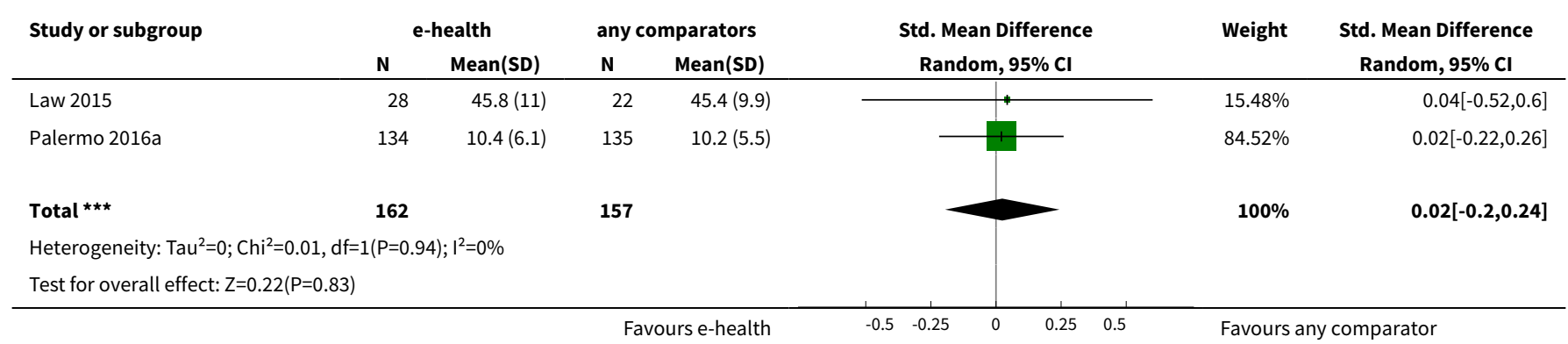

Analysis 1.5. Comparison $1 \mathrm{E}$-health interventions vs any comparator, Outcome 5 Treatment acceptability postintervention.

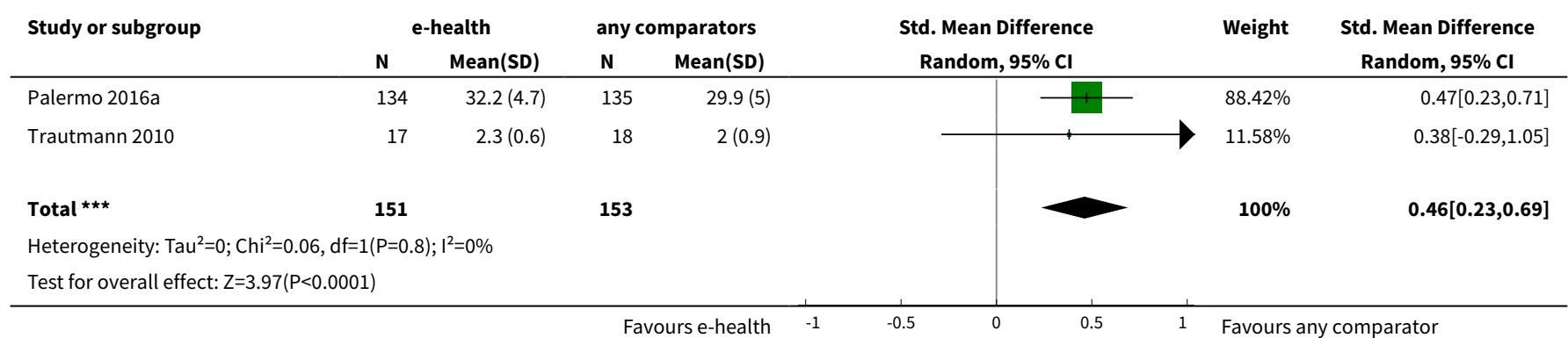

Analysis 1.6. Comparison $1 \mathrm{E}$-health interventions vs any comparator, Outcome 6 Quality of life postintervention.

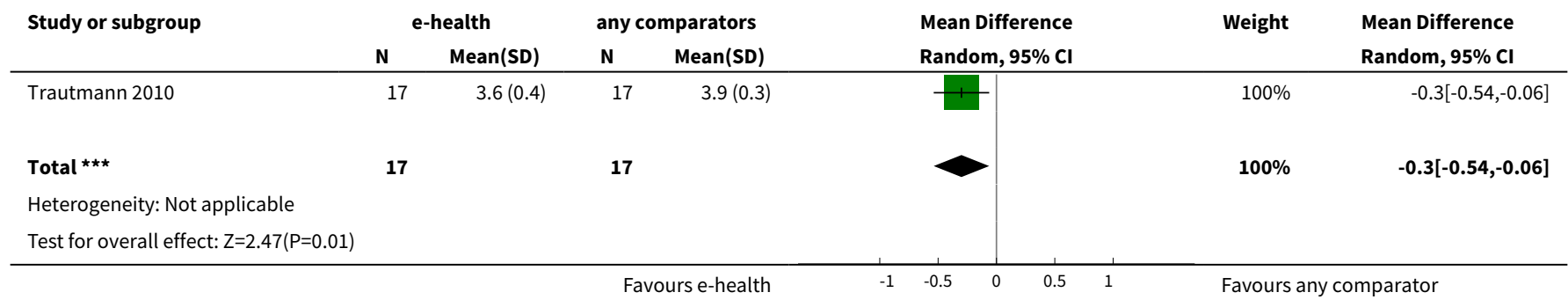


Analysis 1.7. Comparison 1 E-health interventions vs any comparator, Outcome 7 Quality of life 3- to 6-month follow-up.

\begin{tabular}{|c|c|c|c|c|c|c|c|}
\hline \multirow[t]{2}{*}{ Study or subgroup } & \multicolumn{2}{|c|}{ e-health } & \multicolumn{2}{|c|}{ any comparators } & \multirow{2}{*}{$\begin{array}{l}\text { Mean Difference } \\
\text { Random, } 95 \% \mathrm{Cl}\end{array}$} & \multirow[t]{2}{*}{ Weight } & \multirow{2}{*}{$\begin{array}{l}\text { Mean Difference } \\
\text { Random, } 95 \% \mathrm{Cl}\end{array}$} \\
\hline & $\mathbf{N}$ & $\operatorname{Mean}(S D)$ & $\mathbf{N}$ & $\operatorname{Mean}(S D)$ & & & \\
\hline Trautmann 2010 & 12 & $3.9(0.4)$ & 10 & $3.8(0.3)$ & & $100 \%$ & $0.1[-0.19,0.39]$ \\
\hline 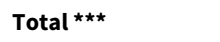 & 12 & & 10 & & & $100 \%$ & $0.1[-0.19,0.39]$ \\
\hline \multicolumn{8}{|c|}{ Heterogeneity: Not applicable } \\
\hline \multicolumn{8}{|c|}{ Test for overall effect: $Z=0.67(P=0.5)$} \\
\hline
\end{tabular}

Analysis 1.8. Comparison 1 E-health interventions vs any comparator, Outcome 8 Functioning postintervention.

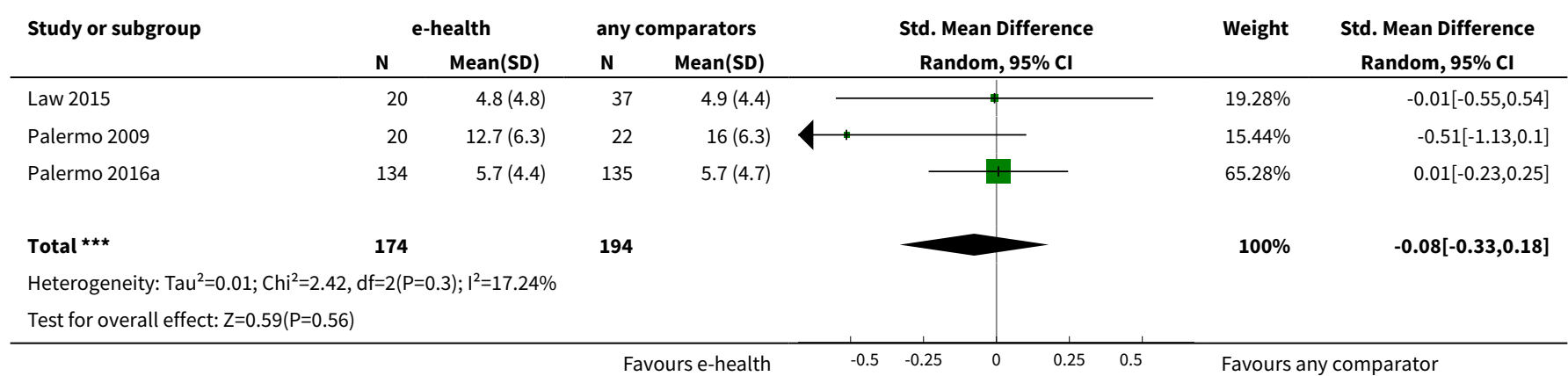

Analysis 1.9. Comparison 1 E-health interventions vs any comparator, Outcome 9 Functioning 3- to 6-month follow-up.

\begin{tabular}{|c|c|c|c|c|c|c|c|}
\hline \multirow[t]{2}{*}{ Study or subgroup } & \multicolumn{2}{|c|}{ e-health } & \multicolumn{2}{|c|}{ any comparators } & \multirow{2}{*}{$\begin{array}{c}\text { Std. Mean Difference } \\
\text { Random, } 95 \% \mathrm{Cl}\end{array}$} & \multirow[t]{2}{*}{ Weight } & \multirow{2}{*}{$\begin{array}{c}\text { Std. Mean Difference } \\
\text { Random, } 95 \% \mathrm{Cl}\end{array}$} \\
\hline & $\mathbf{N}$ & Mean(SD) & $\mathbf{N}$ & Mean(SD) & & & \\
\hline Law 2015 & 28 & $5.2(5)$ & 22 & $5.3(4.6)$ & & $15.52 \%$ & $-0.02[-0.57,0.54]$ \\
\hline Palermo 2016a & 134 & $5.5(4.3)$ & 135 & $6.2(5)$ & & $84.48 \%$ & $-0.15[-0.39,0.09]$ \\
\hline 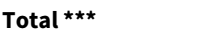 & 162 & & 157 & & & $100 \%$ & $-0.13[-0.35,0.09]$ \\
\hline \multicolumn{8}{|c|}{ Heterogeneity: $\mathrm{Tau}^{2}=0 ; \mathrm{Chi}^{2}=0.19, \mathrm{df}=1(\mathrm{P}=0.66) ; \mathrm{I}^{2}=0 \%$} \\
\hline
\end{tabular}

Analysis 1.10. Comparison 1 E-health interventions vs any comparator, Outcome 10 Status of long-term physical condition postintervention.

\begin{tabular}{|c|c|c|c|c|c|c|c|}
\hline \multirow{2}{*}{ Study or subgroup } & \multicolumn{2}{|c|}{ e-health } & \multicolumn{2}{|c|}{ any comparators } & \multirow{2}{*}{$\begin{array}{c}\text { Std. Mean Difference } \\
\text { Random, } 95 \% \mathrm{CI}\end{array}$} & \multirow[t]{2}{*}{ Weight } & \multirow{2}{*}{$\begin{array}{c}\text { Std. Mean Difference } \\
\text { Random, } 95 \% \mathrm{Cl}\end{array}$} \\
\hline & $\mathbf{N}$ & $\operatorname{Mean}(\mathrm{SD})$ & $\mathbf{N}$ & Mean(SD) & & & \\
\hline Law 2015 & 40 & $4.6(2.1)$ & 37 & $4.7(2.2)$ & \begin{tabular}{l|l} 
& \\
\end{tabular} & $16.69 \%$ & $-0.03[-0.48,0.42]$ \\
\hline Newcombe 2012 & 19 & $83.2(17.2)$ & 20 & $72(32.5)$ & - & $8.26 \%$ & $0.42[-0.22,1.05]$ \\
\hline
\end{tabular}




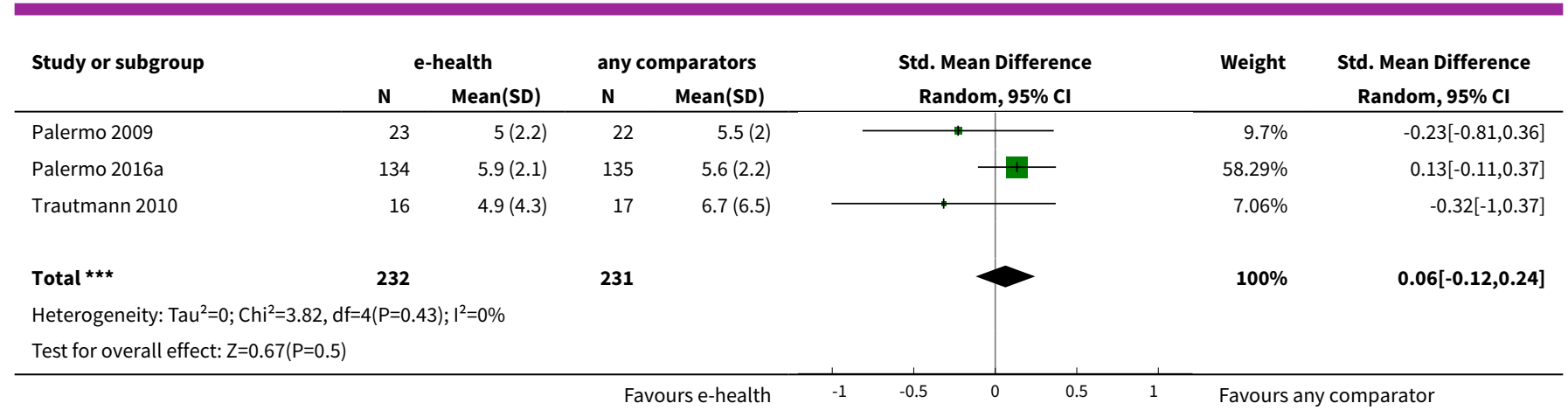

Analysis 1.11. Comparison 1 E-health interventions vs any comparator, Outcome 11 Status of long-term physical condition 3- to 6-month follow-up.

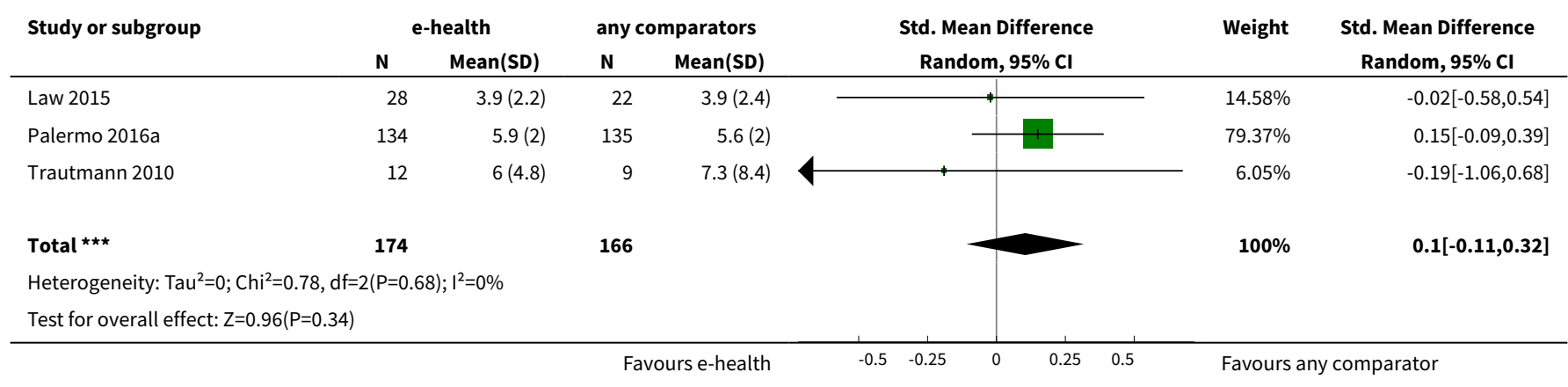

\section{Comparison 2. E-health interventions vs attention placebo}

\begin{tabular}{|c|c|c|c|c|}
\hline Outcome or subgroup title & No. of studies & $\begin{array}{l}\text { No. of partici- } \\
\text { pants }\end{array}$ & Statistical method & Effect size \\
\hline 1 Depression postintervention & 2 & 304 & $\begin{array}{l}\text { Std. Mean Difference (IV, Ran- } \\
\text { dom, } 95 \% \mathrm{CI} \text { ) }\end{array}$ & $0.11[-0.11,0.34]$ \\
\hline $\begin{array}{l}2 \text { Depression 3- to 6-month fol- } \\
\text { low-up }\end{array}$ & 2 & 288 & $\begin{array}{l}\text { Std. Mean Difference (IV, Ran- } \\
\text { dom, } 95 \% \mathrm{Cl} \text { ) }\end{array}$ & $0.02[-0.21,0.25]$ \\
\hline 3 Anxiety postintervention & 1 & 269 & $\begin{array}{l}\text { Mean Difference (IV, Random, } \\
95 \% \mathrm{CI} \text { ) }\end{array}$ & $-0.29[-1.73,1.15]$ \\
\hline 4 Anxiety 3- to 6-month follow-up & 1 & 269 & $\begin{array}{l}\text { Mean Difference (IV, Random, } \\
95 \% \mathrm{CI} \text { ) }\end{array}$ & $0.12[-1.27,1.51]$ \\
\hline $\begin{array}{l}5 \text { Treatment acceptability postin- } \\
\text { tervention }\end{array}$ & 2 & 304 & $\begin{array}{l}\text { Std. Mean Difference (IV, Ran- } \\
\text { dom, } 95 \% \mathrm{CI} \text { ) }\end{array}$ & $0.46[0.23,0.69]$ \\
\hline 6 Quality of life postintervention & 1 & 34 & $\begin{array}{l}\text { Mean Difference (IV, Random, } \\
95 \% \mathrm{CI} \text { ) }\end{array}$ & $-0.30[-0.54,-0.06]$ \\
\hline 7 Quality of life 6-month follow-up & 1 & 22 & $\begin{array}{l}\text { Mean Difference (IV, Random, } \\
95 \% \mathrm{CI} \text { ) }\end{array}$ & $0.10[-0.19,0.39]$ \\
\hline
\end{tabular}




\begin{tabular}{|c|c|c|c|c|}
\hline Outcome or subgroup title & No. of studies & $\begin{array}{l}\text { No. of partici- } \\
\text { pants }\end{array}$ & Statistical method & Effect size \\
\hline 8 Functioning postintervention & 1 & 269 & $\begin{array}{l}\text { Mean Difference (IV, Random, } \\
95 \% \mathrm{CI} \text { ) }\end{array}$ & $0.03[-1.05,1.11]$ \\
\hline $\begin{array}{l}9 \text { Functioning 3- to 6-month fol- } \\
\text { low-up }\end{array}$ & 1 & 269 & $\begin{array}{l}\text { Mean Difference (IV, Random, } \\
95 \% \mathrm{CI} \text { ) }\end{array}$ & $-0.72[-1.84,0.40]$ \\
\hline $\begin{array}{l}10 \text { Status of long-term physical } \\
\text { condition postintervention }\end{array}$ & 2 & 302 & $\begin{array}{l}\text { Std. Mean Difference (IV, Ran- } \\
\text { dom, } 95 \% \mathrm{Cl} \text { ) }\end{array}$ & $0.03[-0.34,0.40]$ \\
\hline $\begin{array}{l}11 \text { Status of long-term physical } \\
\text { condition 3- to 6-month follow-up }\end{array}$ & 2 & 290 & $\begin{array}{l}\text { Std. Mean Difference (IV, Ran- } \\
\text { dom, } 95 \% \mathrm{Cl} \text { ) }\end{array}$ & $0.13[-0.10,0.36]$ \\
\hline
\end{tabular}

Analysis 2.1. Comparison $2 \mathrm{E}$-health interventions vs attention placebo, Outcome 1 Depression postintervention.

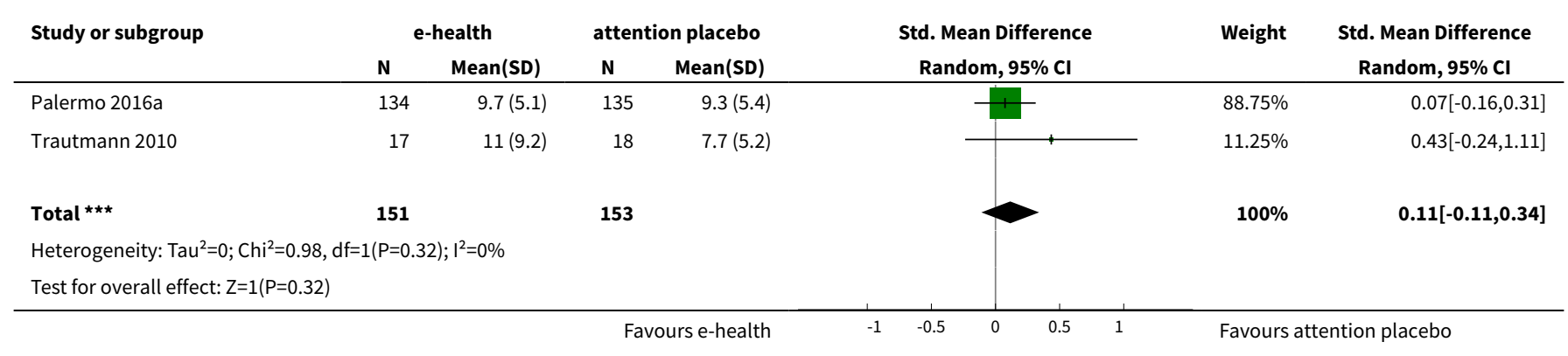

\section{Analysis 2.2. Comparison $2 \mathrm{E}$-health interventions vs attention} placebo, Outcome 2 Depression 3- to 6-month follow-up.

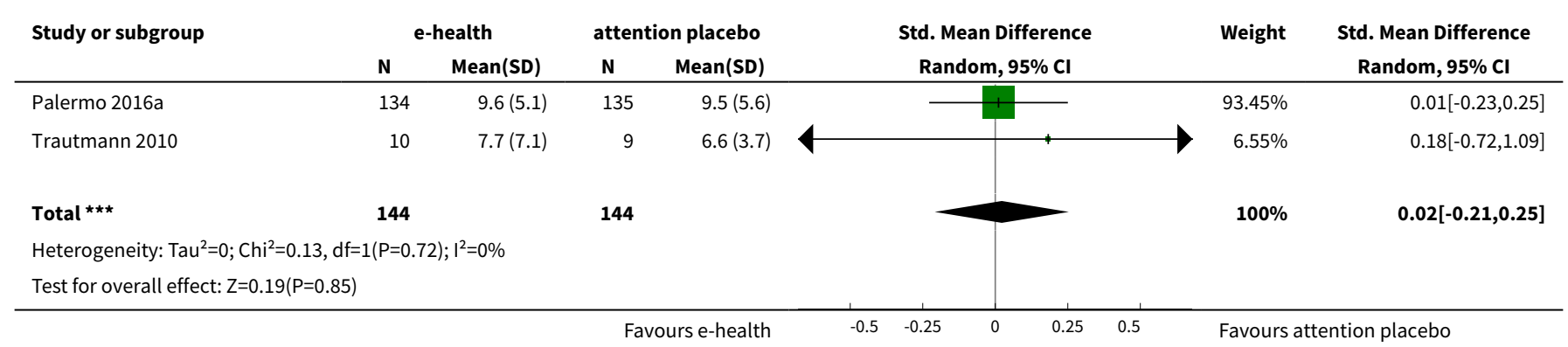

\section{Analysis 2.3. Comparison $2 \mathrm{E}$-health interventions vs attention placebo, Outcome 3 Anxiety postintervention.}

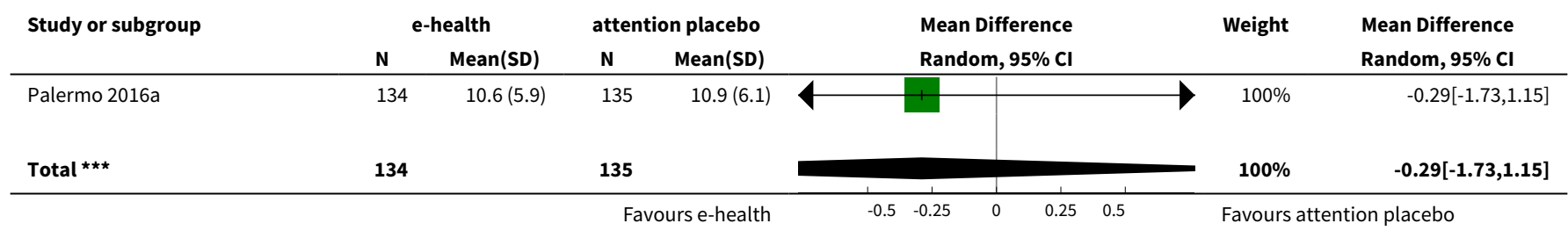




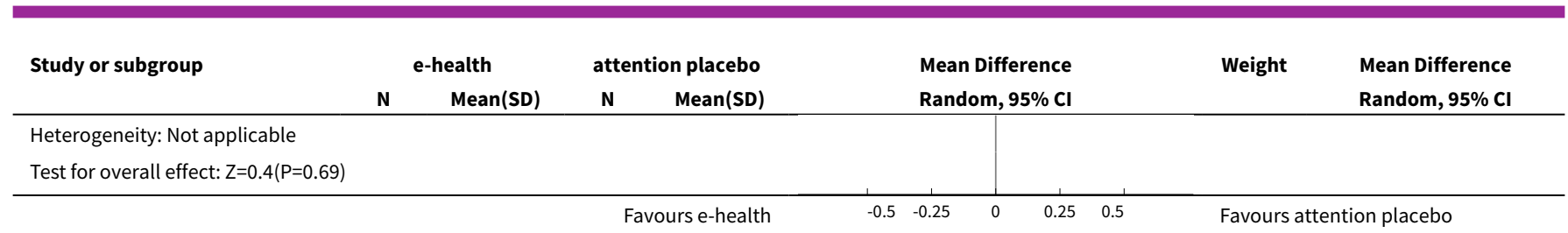

Analysis 2.4. Comparison $2 \mathrm{E}$-health interventions vs attention placebo, Outcome 4 Anxiety 3- to 6-month follow-up.

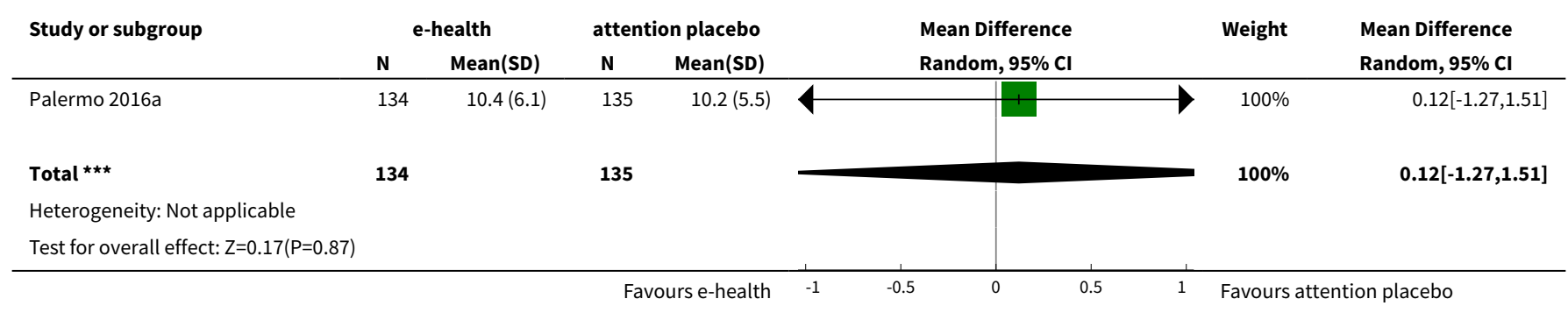

Analysis 2.5. Comparison $2 \mathrm{E}$-health interventions vs attention placebo, Outcome 5 Treatment acceptability postintervention.

\begin{tabular}{|c|c|c|c|c|c|c|c|}
\hline \multirow{3}{*}{$\begin{array}{l}\text { Study or subgroup } \\
\text { Palermo } 2016 a\end{array}$} & \multicolumn{2}{|c|}{ e-health } & \multicolumn{2}{|c|}{ attention placebo } & \multirow{2}{*}{$\begin{array}{c}\text { Std. Mean Difference } \\
\text { Random, } 95 \% \mathrm{Cl}\end{array}$} & \multirow[t]{2}{*}{ Weight } & \multirow{2}{*}{$\begin{array}{c}\text { Std. Mean Difference } \\
\text { Random, } 95 \% \mathrm{CI}\end{array}$} \\
\hline & $\mathbf{N}$ & Mean(SD) & $\mathbf{N}$ & Mean(SD) & & & \\
\hline & 134 & $32.2(4.7)$ & 135 & $29.9(5)$ & & $88.42 \%$ & $0.47[0.23,0.71]$ \\
\hline Total $\star \star \star ~$ & 151 & & 153 & & & $100 \%$ & $0.46[0.23,0.69]$ \\
\hline \multicolumn{8}{|c|}{ Heterogeneity: Tau $^{2}=0 ; \mathrm{Chi}^{2}=0.06, \mathrm{df}=1(\mathrm{P}=0.8) ; \mathrm{I}^{2}=0 \%$} \\
\hline \multicolumn{8}{|c|}{ Test for overall effect: $Z=3.97(P<0.0001)$} \\
\hline
\end{tabular}

Analysis 2.6. Comparison $2 \mathrm{E}$-health interventions vs attention placebo, Outcome 6 Quality of life postintervention.

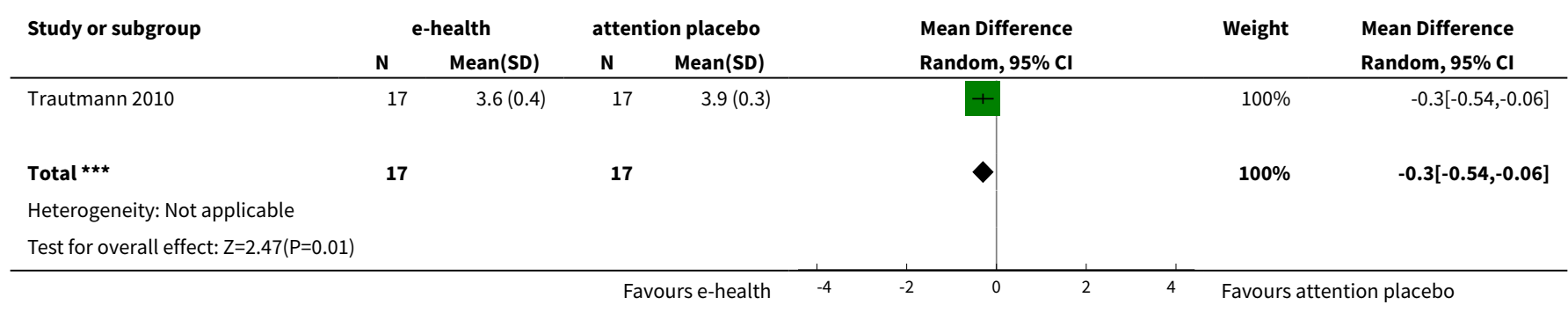


Analysis 2.7. Comparison $2 \mathrm{E}$-health interventions vs attention placebo, Outcome 7 Quality of life 6-month follow-up.

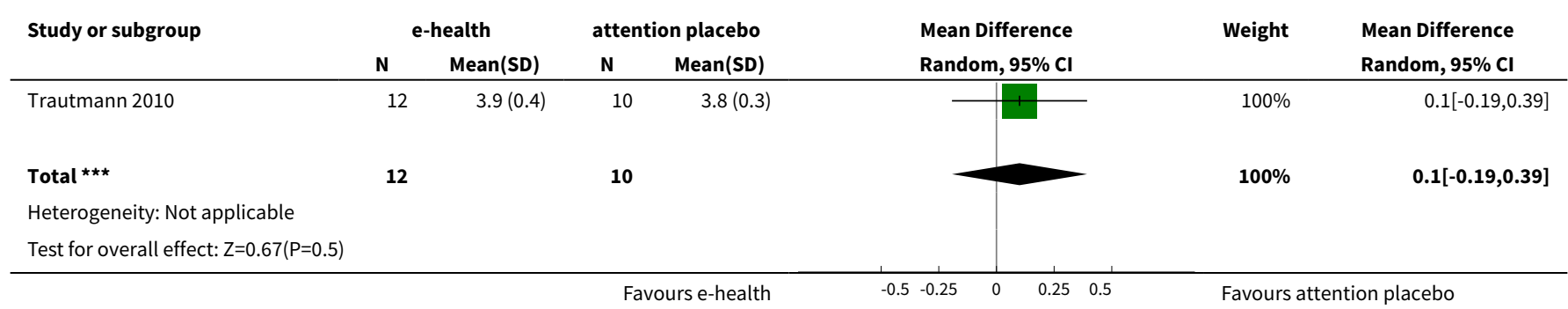

Analysis 2.8. Comparison $2 \mathrm{E}$-health interventions vs attention placebo, Outcome 8 Functioning postintervention.

\begin{tabular}{|c|c|c|c|c|c|c|c|}
\hline \multirow[t]{2}{*}{ Study or subgroup } & \multicolumn{2}{|c|}{ e-health } & \multicolumn{2}{|c|}{ attention placebo } & \multirow{2}{*}{$\begin{array}{l}\text { Mean Difference } \\
\text { Random, } 95 \% \mathrm{Cl}\end{array}$} & \multirow[t]{2}{*}{ Weight } & \multirow{2}{*}{$\begin{array}{l}\text { Mean Difference } \\
\text { Random, } 95 \% \mathrm{Cl}\end{array}$} \\
\hline & $\mathbf{N}$ & Mean(SD) & $\mathbf{N}$ & Mean(SD) & & & \\
\hline Palermo 2016a & 134 & $5.7(4.4)$ & 135 & $5.7(4.7)$ & & $100 \%$ & $0.03[-1.05,1.11]$ \\
\hline 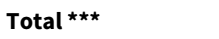 & 134 & & 135 & & & $100 \%$ & $0.03[-1.05,1.11]$ \\
\hline \multicolumn{8}{|c|}{ Heterogeneity: Not applicable } \\
\hline \multicolumn{8}{|c|}{ Test for overall effect: $Z=0.05(P=0.96)$} \\
\hline
\end{tabular}

\section{Analysis 2.9. Comparison $2 \mathrm{E}$-health interventions vs attention} placebo, Outcome 9 Functioning 3- to 6-month follow-up.

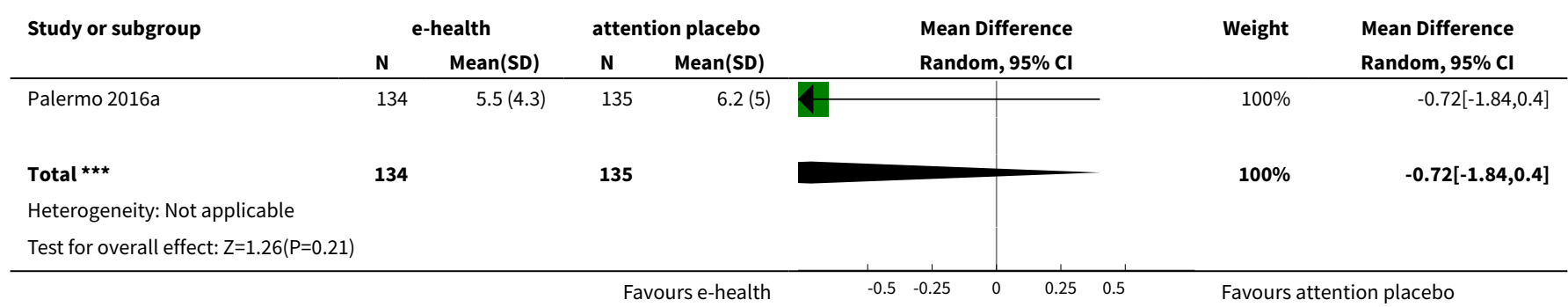

Analysis 2.10. Comparison $2 \mathrm{E}$-health interventions vs attention placebo, Outcome 10 Status of long-term physical condition postintervention.

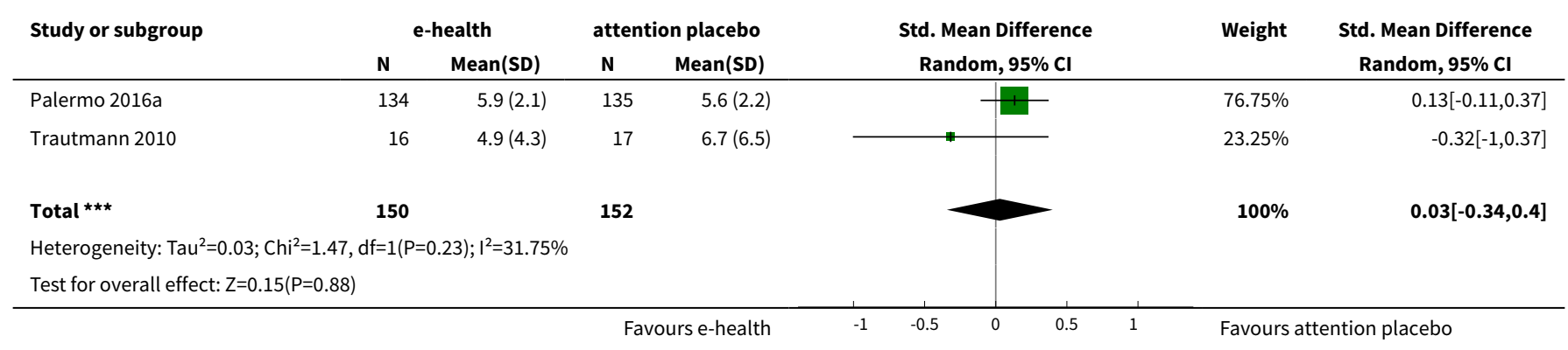


Analysis 2.11. Comparison 2 E-health interventions vs attention placebo, Outcome 11 Status of long-term physical condition 3- to 6-month follow-up.

\begin{tabular}{|c|c|c|c|c|c|c|c|}
\hline \multirow[t]{2}{*}{ Study or subgroup } & \multicolumn{2}{|c|}{ e-health } & \multicolumn{2}{|c|}{ attention placebo } & \multirow{2}{*}{$\begin{array}{c}\text { Std. Mean Difference } \\
\text { Random, } 95 \% \mathrm{Cl}\end{array}$} & \multirow[t]{2}{*}{ Weight } & \multirow{2}{*}{$\begin{array}{c}\text { Std. Mean Difference } \\
\text { Random, } 95 \% \mathrm{Cl}\end{array}$} \\
\hline & $\mathbf{N}$ & Mean(SD) & $\mathbf{N}$ & Mean(SD) & & & \\
\hline Palermo 2016a & 134 & $5.9(2)$ & 135 & $5.6(2)$ & 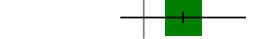 & $92.91 \%$ & $0.15[-0.09,0.39]$ \\
\hline Trautmann 2010 & 12 & $6(4.8)$ & 9 & $7.3(8.4)$ & & $7.09 \%$ & $-0.19[-1.06,0.68]$ \\
\hline 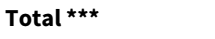 & 146 & & 144 & & & $100 \%$ & $0.13[-0.1,0.36]$ \\
\hline \multicolumn{8}{|c|}{ Heterogeneity: $\operatorname{Tau}^{2}=0 ; \mathrm{Chi}^{2}=0.55, \mathrm{df}=1(\mathrm{P}=0.46) ;\left.\right|^{2}=0 \%$} \\
\hline & & & & urs e-health & $-0.5-0.25$ & Favours & htion placebo \\
\hline
\end{tabular}

\section{Comparison 3. E-health interventions vs treatment as usual (TAU)}

\begin{tabular}{|c|c|c|c|c|}
\hline Outcome or subgroup title & No. of studies & $\begin{array}{l}\text { No. of partici- } \\
\text { pants }\end{array}$ & Statistical method & Effect size \\
\hline 1 Depression postintervention & 1 & 50 & $\begin{array}{l}\text { Mean Difference (IV, Random, } \\
95 \% \mathrm{CI} \text { ) }\end{array}$ & $-1.18[-6.60,4.24]$ \\
\hline 2 Depression 3-month follow-up & 1 & 51 & $\begin{array}{l}\text { Mean Difference (IV, Random, } \\
95 \% \mathrm{CI} \text { ) }\end{array}$ & $1.01[-3.39,5.41]$ \\
\hline 3 Anxiety postintervention & 1 & 55 & $\begin{array}{l}\text { Mean Difference (IV, Random, } \\
95 \% \mathrm{CI} \text { ) }\end{array}$ & $-1.99[-7.31,3.33]$ \\
\hline 4 Anxiety 3-month follow-up & 1 & 50 & $\begin{array}{l}\text { Mean Difference (IV, Random, } \\
95 \% \mathrm{CI} \text { ) }\end{array}$ & $0.46[-5.34,6.26]$ \\
\hline 5 Functioning postintervention & 1 & 57 & $\begin{array}{l}\text { Mean Difference (IV, Random, } \\
95 \% \mathrm{CI} \text { ) }\end{array}$ & $-0.03[-2.56,2.50]$ \\
\hline 6 Functioning 3-month follow-up & 1 & 50 & $\begin{array}{l}\text { Mean Difference (IV, Random, } \\
95 \% \mathrm{CI} \text { ) }\end{array}$ & $-0.08[-2.76,2.60]$ \\
\hline $\begin{array}{l}7 \text { Status of long-term physical con- } \\
\text { dition postintervention }\end{array}$ & 1 & 77 & $\begin{array}{l}\text { Mean Difference (IV, Random, } \\
95 \% \mathrm{CI} \text { ) }\end{array}$ & $-0.07[-1.05,0.91]$ \\
\hline $\begin{array}{l}8 \text { Status of long-term physical con- } \\
\text { dition at 3- to 6-month follow-up }\end{array}$ & 1 & 50 & $\begin{array}{l}\text { Mean Difference (IV, Random, } \\
95 \% \mathrm{CI} \text { ) }\end{array}$ & $-0.05[-1.34,1.24]$ \\
\hline
\end{tabular}

Analysis 3.1. Comparison 3 E-health interventions vs treatment as usual (TAU), Outcome 1 Depression postintervention.

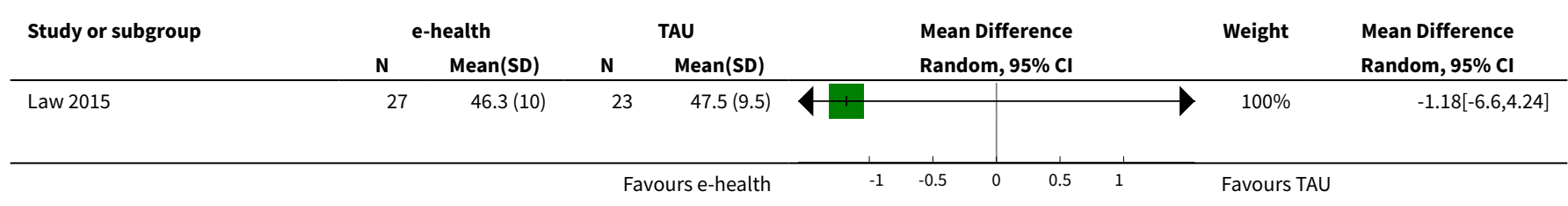




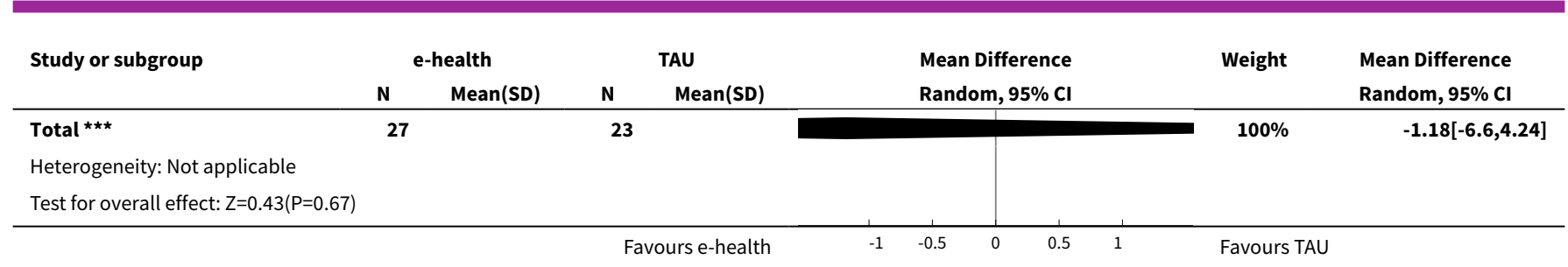

\section{Analysis 3.2. Comparison $3 \mathrm{E}$-health interventions vs treatment} as usual (TAU), Outcome 2 Depression 3-month follow-up.

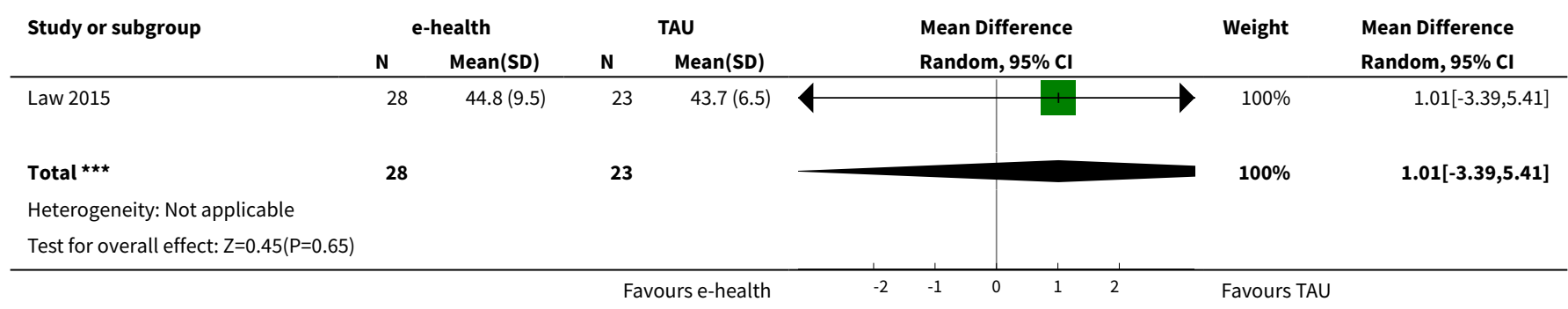

Analysis 3.3. Comparison 3 E-health interventions vs treatment as usual (TAU), Outcome 3 Anxiety postintervention.

\begin{tabular}{|c|c|c|c|c|c|c|c|}
\hline \multirow{3}{*}{$\begin{array}{l}\text { Study or subgroup } \\
\text { Law } 2015\end{array}$} & \multicolumn{2}{|c|}{ e-health } & \multicolumn{2}{|c|}{ TAU } & \multirow{2}{*}{$\begin{array}{l}\text { Mean Difference } \\
\text { Random, 95\% Cl }\end{array}$} & \multirow[t]{2}{*}{ Weight } & \multirow{2}{*}{$\begin{array}{l}\text { Mean Difference } \\
\text { Random, } 95 \% \mathrm{Cl}\end{array}$} \\
\hline & $\mathbf{N}$ & Mean(SD) & $\mathbf{N}$ & Mean(SD) & & & \\
\hline & 30 & $46.3(9)$ & 25 & $48.3(10.8)$ & & $100 \%$ & $-1.99[-7.31,3.33]$ \\
\hline 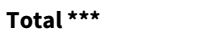 & 30 & & 25 & & & $100 \%$ & $-1.99[-7.31,3.33]$ \\
\hline \multicolumn{8}{|c|}{ Heterogeneity: $\operatorname{Tau}^{2}=0 ; \mathrm{Chi}^{2}=0, \mathrm{df}=0(\mathrm{P}<0.0001) ; \mathrm{I}^{2}=100 \%$} \\
\hline \multicolumn{8}{|c|}{ Test for overall effect: $Z=0.73(P=0.46)$} \\
\hline
\end{tabular}

\section{Analysis 3.4. Comparison $3 \mathrm{E}$-health interventions vs treatment} as usual (TAU), Outcome 4 Anxiety 3-month follow-up.

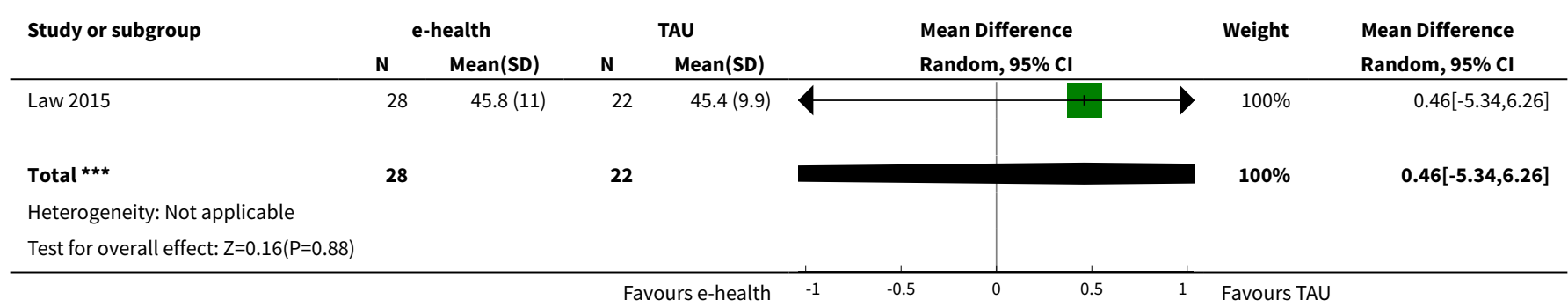


Analysis 3.5. Comparison $3 \mathrm{E}$-health interventions vs treatment as usual (TAU), Outcome 5 Functioning postintervention.

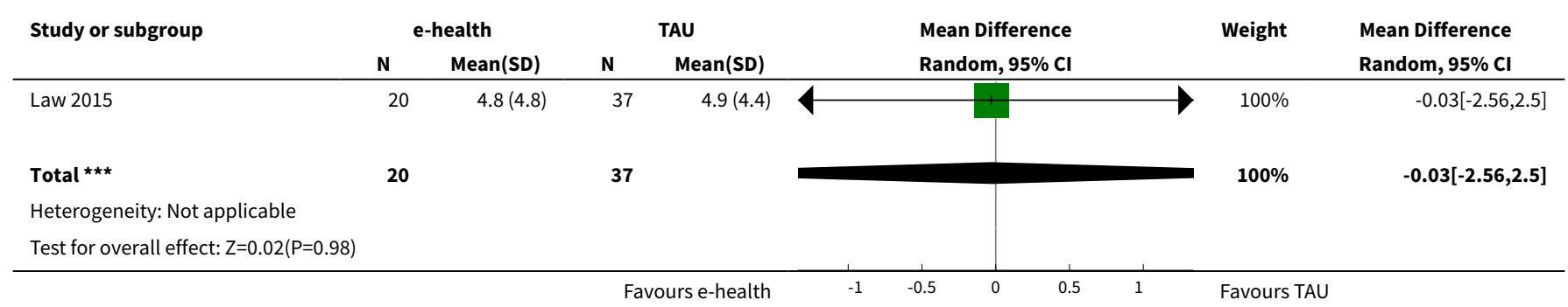

Analysis 3.6. Comparison $3 \mathrm{E}$-health interventions vs treatment as usual (TAU), Outcome 6 Functioning 3-month follow-up.

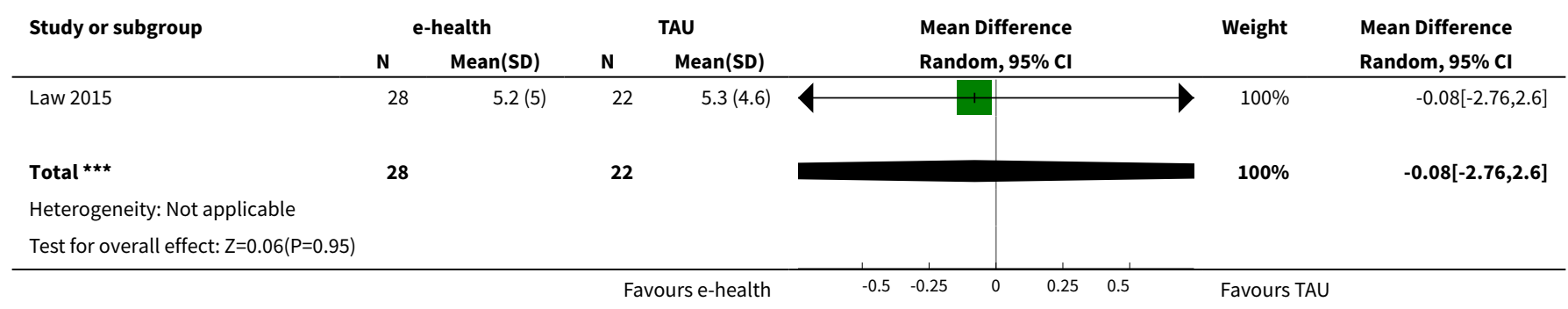

Analysis 3.7. Comparison $3 \mathrm{E}$-health interventions vs treatment as usual (TAU), Outcome 7 Status of long-term physical condition postintervention.

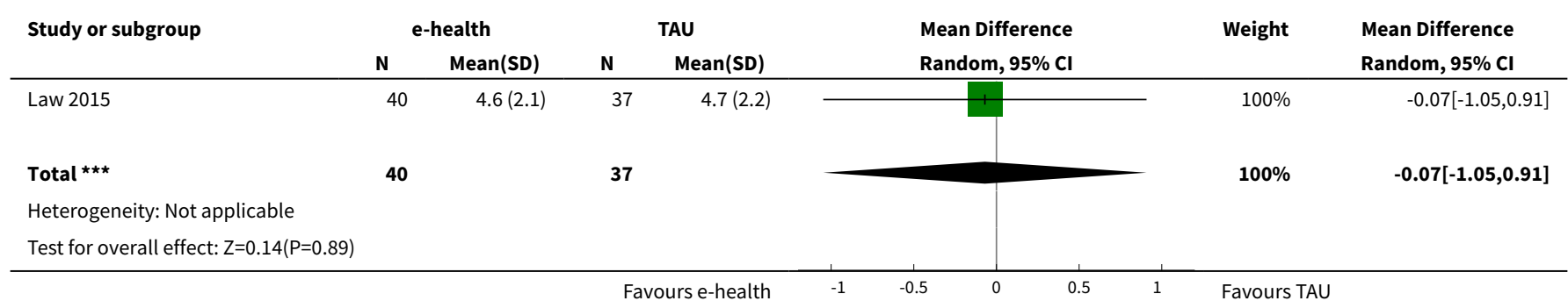

Analysis 3.8. Comparison $3 \mathrm{E}$-health interventions vs treatment as usual (TAU), Outcome 8 Status of long-term physical condition at 3- to 6-month follow-up.

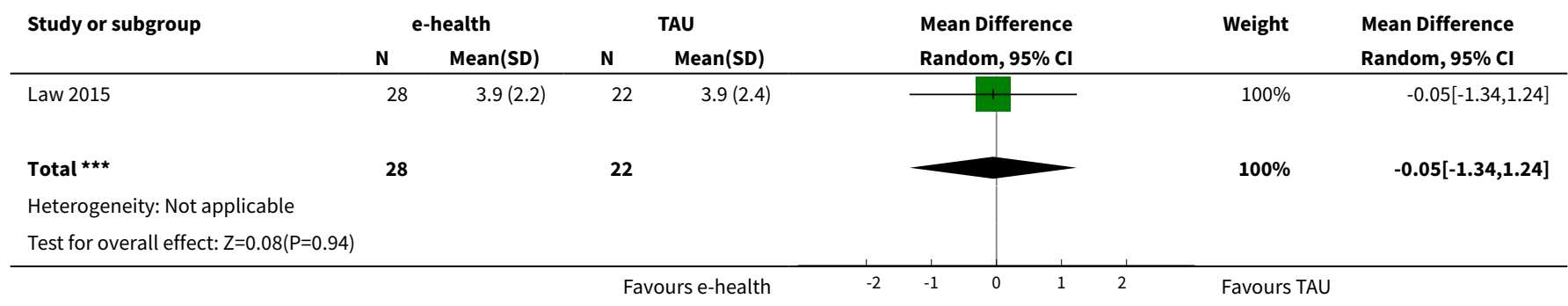


Comparison 4. E-health interventions vs waiting list

\begin{tabular}{lllll}
\hline Outcome or subgroup title & No. of studies & $\begin{array}{l}\text { No. of partici- } \\
\text { pants }\end{array}$ & Statistical method & Effect size \\
\hline 1 Depression postintervention & 2 & 87 & $\begin{array}{l}\text { Std. Mean Difference (IV, Ran- } \\
\text { dom, 95\% Cl) }\end{array}$ & $-0.40[-0.91,0.11]$ \\
\hline 2 Functioning postintervention & 1 & 48 & $\begin{array}{l}\text { Mean Difference (IV, Random, } \\
95 \% \text { Cl) }\end{array}$ & $-3.31[-6.90,0.28]$ \\
\hline $\begin{array}{l}\text { 3 Status of long-term physical con- } \\
\text { dition postintervention }\end{array}$ & 2 & 84 & $\begin{array}{l}\text { Std. Mean Difference (IV, Ran- } \\
\text { dom, 95\% Cl) }\end{array}$ & 0.08 [-0.55, 0.72] \\
\hline
\end{tabular}

Analysis 4.1. Comparison 4 E-health interventions vs waiting list, Outcome 1 Depression postintervention.

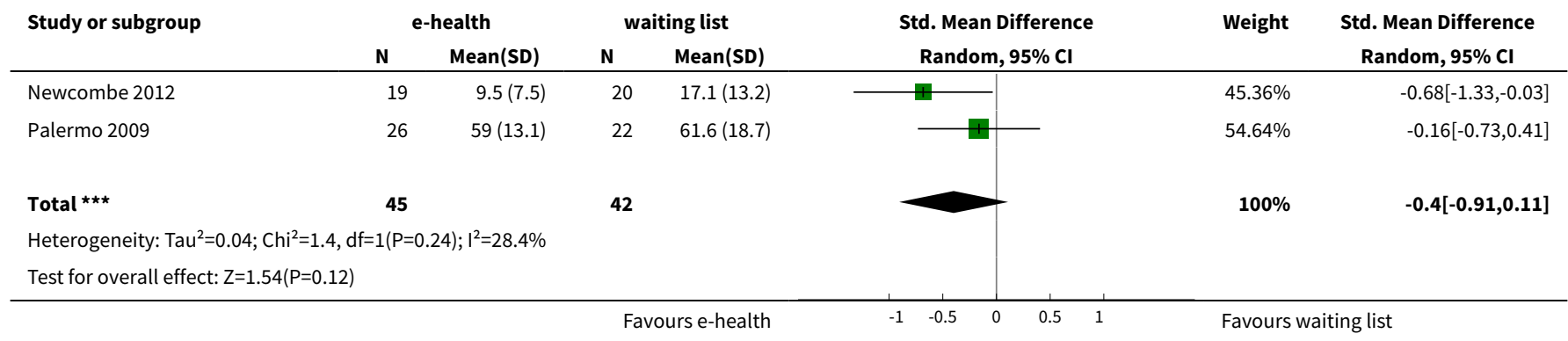

Analysis 4.2. Comparison 4 E-health interventions vs waiting list, Outcome 2 Functioning postintervention.

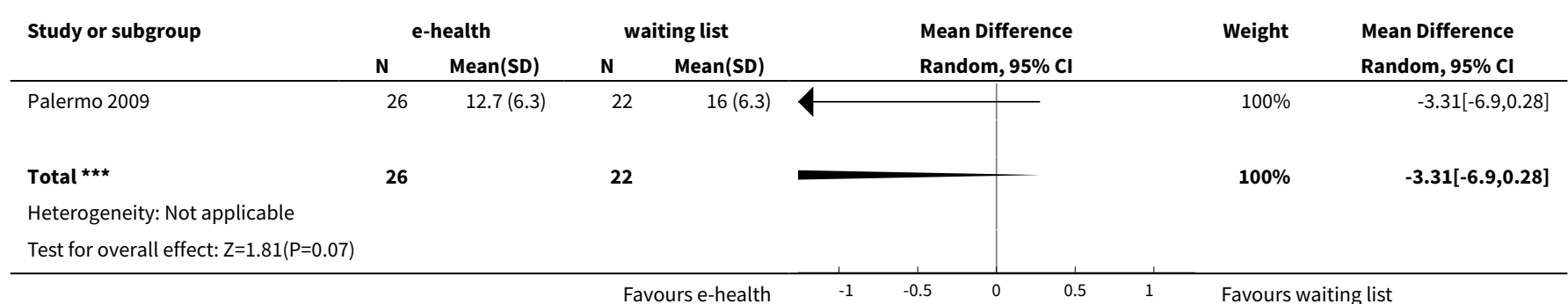

Analysis 4.3. Comparison 4 E-health interventions vs waiting list, Outcome 3 Status of long-term physical condition postintervention.

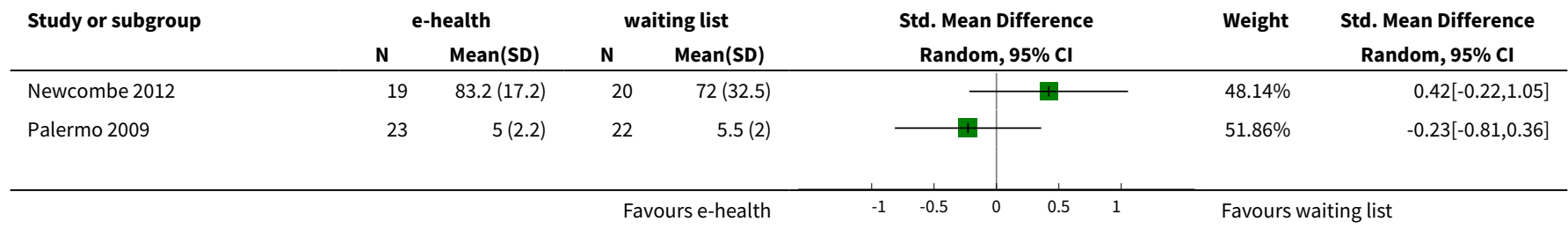




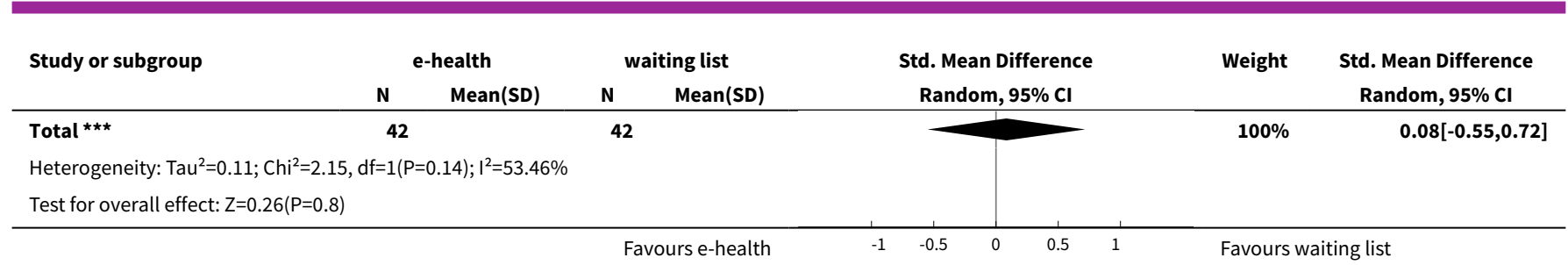

Comparison 5. E-health interventions vs any comparison (by type of therapy)

\begin{tabular}{lllll}
\hline $\begin{array}{l}\text { Outcome or sub- } \\
\text { group title }\end{array}$ & No. of studies & $\begin{array}{l}\text { No. of partici- } \\
\text { pants }\end{array}$ & Statistical method & Effect size \\
\hline $\begin{array}{l}1 \text { Depression postin- } \\
\text { tervention }\end{array}$ & 5 & 441 & Std. Mean Difference (IV, Random, 95\% CI) & $-0.06[-0.35,0.23]$ \\
\hline 1.1 CBT & 4 & 402 & Std. Mean Difference (IV, Random, 95\% CI) & $0.05[-0.14,0.25]$ \\
\hline 1.2 Non-CBT & 1 & 39 & Std. Mean Difference (IV, Random, 95\% CI) & $-0.68[-1.33,-0.04]$ \\
\hline
\end{tabular}

Analysis 5.1. Comparison $5 \mathrm{E}$-health interventions vs any comparison (by type of therapy), Outcome 1 Depression postintervention.

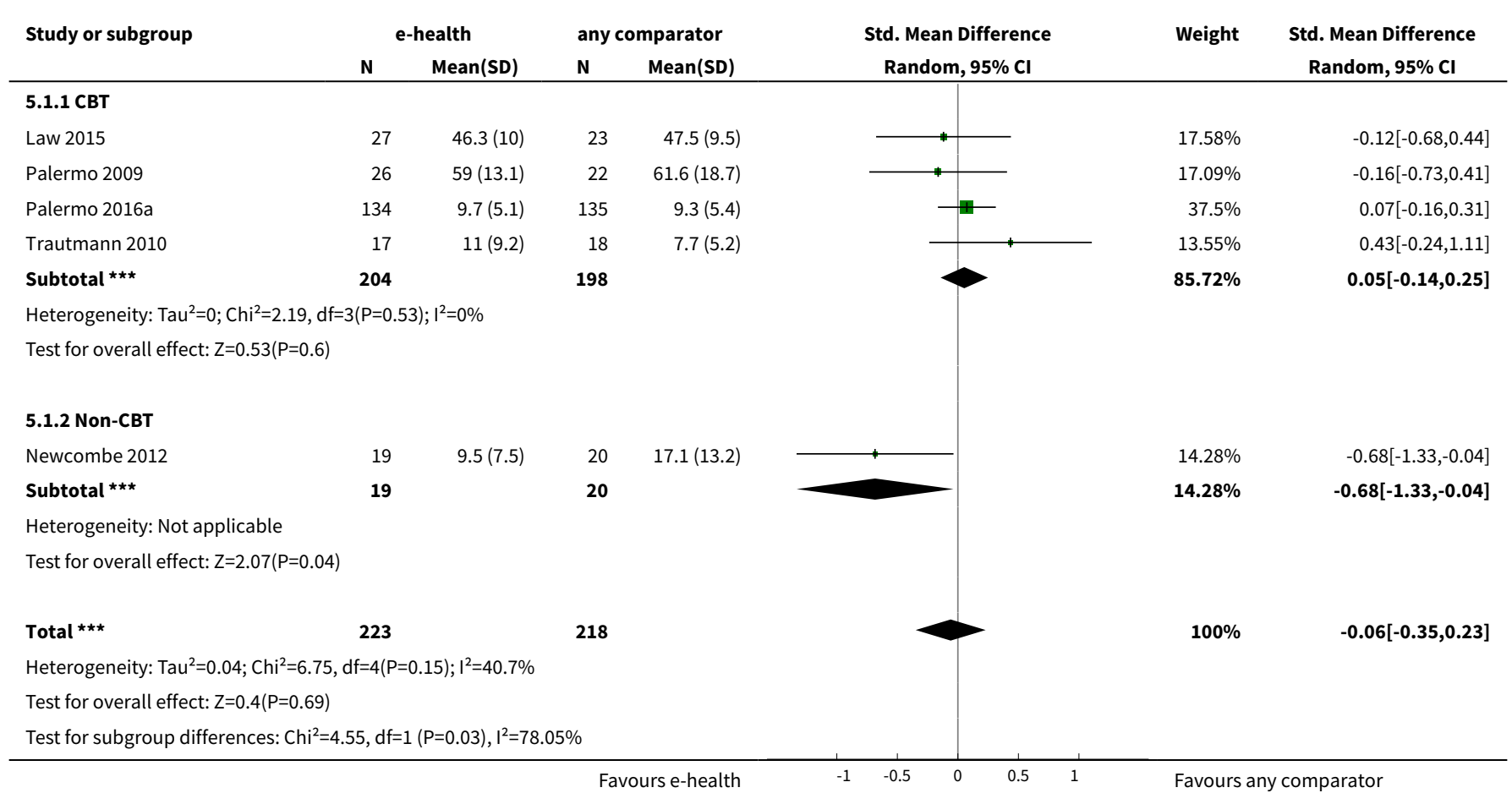


Comparison 6. E-health interventions vs any comparator (by type of comparator)

\begin{tabular}{|c|c|c|c|c|}
\hline $\begin{array}{l}\text { Outcome or subgroup ti- } \\
\text { tle }\end{array}$ & No. of studies & $\begin{array}{l}\text { No. of partici- } \\
\text { pants }\end{array}$ & Statistical method & Effect size \\
\hline $\begin{array}{l}1 \text { Depression postinter- } \\
\text { vention }\end{array}$ & 5 & 441 & $\begin{array}{l}\text { Std. Mean Difference (IV, Random, 95\% } \\
\mathrm{Cl} \text { ) }\end{array}$ & $-0.06[-0.35,0.23]$ \\
\hline 1.1 Attention placebo & 2 & 304 & $\begin{array}{l}\text { Std. Mean Difference (IV, Random, 95\% } \\
\mathrm{CI} \text { ) }\end{array}$ & $0.11[-0.11,0.34]$ \\
\hline $\begin{array}{l}\text { 1.2 Treatment as usual } \\
(\mathrm{TAU})\end{array}$ & 1 & 50 & $\begin{array}{l}\text { Std. Mean Difference (IV, Random, 95\% } \\
\mathrm{CI} \text { ) }\end{array}$ & $-0.12[-0.68,0.44]$ \\
\hline 1.3 Waiting list & 2 & 87 & $\begin{array}{l}\text { Std. Mean Difference (IV, Random, 95\% } \\
\mathrm{CI} \text { ) }\end{array}$ & $-0.40[-0.91,0.11]$ \\
\hline $\begin{array}{l}2 \text { Depression 3- to 6- } \\
\text { month follow-up }\end{array}$ & 3 & 339 & $\begin{array}{l}\text { Std. Mean Difference (IV, Random, 95\% } \\
\mathrm{CI} \text { ) }\end{array}$ & $0.04[-0.18,0.25]$ \\
\hline 2.1 Attention placebo & 2 & 288 & $\begin{array}{l}\text { Std. Mean Difference (IV, Random, 95\% } \\
\mathrm{Cl} \text { ) }\end{array}$ & $0.02[-0.21,0.25]$ \\
\hline 2.2 TAU & 1 & 51 & $\begin{array}{l}\text { Std. Mean Difference (IV, Random, 95\% } \\
\mathrm{CI} \text { ) }\end{array}$ & $0.12[-0.43,0.67]$ \\
\hline 3 Anxiety postintervention & 2 & 324 & $\begin{array}{l}\text { Std. Mean Difference (IV, Random, 95\% } \\
\mathrm{CI} \text { ) }\end{array}$ & $-0.07[-0.29,0.14]$ \\
\hline 3.1 Attention placebo & 1 & 269 & $\begin{array}{l}\text { Std. Mean Difference (IV, Random, 95\% } \\
\mathrm{Cl} \text { ) }\end{array}$ & $-0.05[-0.29,0.19]$ \\
\hline $\begin{array}{l}\text { 3.2 Treatment as usual } \\
\text { (TAU) }\end{array}$ & 1 & 55 & $\begin{array}{l}\text { Std. Mean Difference (IV, Random, 95\% } \\
\mathrm{CI} \text { ) }\end{array}$ & $-0.20[-0.73,0.33]$ \\
\hline $\begin{array}{l}4 \text { Anxiety 3- to 6-month } \\
\text { follow-up }\end{array}$ & 2 & 319 & $\begin{array}{l}\text { Std. Mean Difference (IV, Random, 95\% } \\
\mathrm{Cl} \text { ) }\end{array}$ & $0.02[-0.20,0.24]$ \\
\hline 4.1 Attention placebo & 1 & 269 & $\begin{array}{l}\text { Std. Mean Difference (IV, Random, 95\% } \\
\mathrm{CI} \text { ) }\end{array}$ & $0.02[-0.22,0.26]$ \\
\hline $\begin{array}{l}\text { 4.2 Treatment as usual } \\
\text { (TAU) }\end{array}$ & 1 & 50 & $\begin{array}{l}\text { Std. Mean Difference (IV, Random, 95\% } \\
\mathrm{CI} \text { ) }\end{array}$ & $0.04[-0.52,0.60]$ \\
\hline $\begin{array}{l}5 \text { Treatment acceptability } \\
\text { postintervention }\end{array}$ & 1 & 35 & $\begin{array}{l}\text { Std. Mean Difference (IV, Random, 95\% } \\
\mathrm{Cl} \text { ) }\end{array}$ & $0.38[-0.29,1.05]$ \\
\hline 5.1 Attention placebo & 1 & 35 & $\begin{array}{l}\text { Std. Mean Difference (IV, Random, 95\% } \\
\mathrm{Cl} \text { ) }\end{array}$ & $0.38[-0.29,1.05]$ \\
\hline
\end{tabular}


Analysis 6.1. Comparison 6 E-health interventions vs any comparator (by type of comparator), Outcome 1 Depression postintervention.

\begin{tabular}{|c|c|c|c|c|c|c|c|}
\hline \multirow[t]{2}{*}{ Study or subgroup } & \multicolumn{2}{|c|}{ e-health } & \multicolumn{2}{|c|}{ comparator } & \multirow{2}{*}{$\begin{array}{c}\text { Std. Mean Difference } \\
\text { Random, } 95 \% \mathrm{Cl}\end{array}$} & \multirow[t]{2}{*}{ Weight } & \multirow{2}{*}{$\begin{array}{c}\text { Std. Mean Difference } \\
\text { Random, } 95 \% \mathrm{Cl}\end{array}$} \\
\hline & $\mathbf{N}$ & Mean(SD) & $\mathbf{N}$ & Mean(SD) & & & \\
\hline \multicolumn{8}{|c|}{ 6.1.1 Attention placebo } \\
\hline Palermo 2016a & 134 & $9.7(5.1)$ & 135 & $9.3(5.4)$ & - & $37.57 \%$ & $0.07[-0.16,0.31]$ \\
\hline Trautmann 2010 & 17 & $11(9.2)$ & 18 & $7.7(5.2)$ & & $13.53 \%$ & $0.43[-0.24,1.11]$ \\
\hline Subtotal $\star \star \star$ & 151 & & 153 & & & $51.11 \%$ & $0.11[-0.11,0.34]$ \\
\hline \multicolumn{8}{|c|}{ Heterogeneity: $\mathrm{Tau}^{2}=0 ; \mathrm{Chi}^{2}=0.98, \mathrm{df}=1(\mathrm{P}=0.32) ; \mathrm{I}^{2}=0 \%$} \\
\hline \multicolumn{8}{|c|}{ 6.1.2 Treatment as usual (TAU) } \\
\hline Law 2015 & 27 & $46.3(10)$ & 23 & $47.5(9.5)$ & & $17.56 \%$ & $-0.12[-0.68,0.44]$ \\
\hline Subtotal $\star \star \star$ & 27 & & 23 & & & $17.56 \%$ & $-0.12[-0.68,0.44]$ \\
\hline \multicolumn{8}{|c|}{ Heterogeneity: Not applicable } \\
\hline \multicolumn{8}{|c|}{ Test for overall effect: $Z=0.42(P=0.68)$} \\
\hline \multicolumn{8}{|l|}{ 6.1.3 Waiting list } \\
\hline Newcombe 2012 & 19 & $9.5(7.5)$ & 20 & $17.1(13.2)$ & + & $14.26 \%$ & $-0.68[-1.33,-0.03]$ \\
\hline Palermo 2009 & 26 & $59(13.1)$ & 22 & $61.6(18.7)$ & 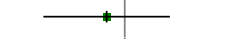 & $17.07 \%$ & $-0.16[-0.73,0.41]$ \\
\hline Subtotal $* \star \star$ & 45 & & 42 & & & $31.33 \%$ & $-0.4[-0.91,0.11]$ \\
\hline \multicolumn{8}{|c|}{ Heterogeneity: $\mathrm{Tau}^{2}=0.04 ; \mathrm{Chi}^{2}=1.4, \mathrm{df}=1(\mathrm{P}=0.24) ; \mathrm{I}^{2}=28.4 \%$} \\
\hline \multicolumn{8}{|c|}{ Test for overall effect: $Z=1.54(P=0.12)$} \\
\hline 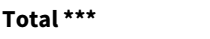 & 223 & & 218 & & & $100 \%$ & $-0.06[-0.35,0.23]$ \\
\hline \multicolumn{8}{|c|}{ Heterogeneity: $\mathrm{Tau}^{2}=0.04 ; \mathrm{Chi}^{2}=6.73, \mathrm{df}=4(\mathrm{P}=0.15) ; \mathrm{I}^{2}=40.54 \%$} \\
\hline \multicolumn{8}{|c|}{ Test for overall effect: $Z=0.4(P=0.69)$} \\
\hline Test for subgroup dif & $53, \mathrm{df}=$ & $P=0.17), I^{2}=4$ & & & & & \\
\hline
\end{tabular}

Analysis 6.2. Comparison 6 E-health interventions vs any comparator (by type of comparator), Outcome 2 Depression 3- to 6-month follow-up.

\begin{tabular}{|c|c|c|c|c|c|c|c|}
\hline \multirow[t]{2}{*}{ Study or subgroup } & \multicolumn{2}{|c|}{ e-health } & \multicolumn{2}{|c|}{ comparator } & \multirow{2}{*}{$\begin{array}{c}\text { Std. Mean Difference } \\
\text { Random, } 95 \% \mathrm{Cl}\end{array}$} & \multirow[t]{2}{*}{ Weight } & \multirow{2}{*}{$\begin{array}{c}\text { Std. Mean Difference } \\
\text { Random, } 95 \% \mathrm{Cl}\end{array}$} \\
\hline & $\mathbf{N}$ & Mean(SD) & $\mathbf{N}$ & Mean(SD) & & & \\
\hline \multicolumn{8}{|c|}{ 6.2.1 Attention placebo } \\
\hline Palermo 2016a & 134 & $9.6(5.1)$ & 135 & $9.5(5.6)$ & & $79.52 \%$ & $0.01[-0.23,0.25]$ \\
\hline Trautmann 2010 & 10 & $7.7(7.1)$ & 9 & $6.6(3.7)$ & & $5.57 \%$ & $0.18[-0.72,1.09]$ \\
\hline 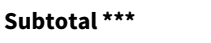 & 144 & & 144 & & & $85.1 \%$ & $0.02[-0.21,0.25]$ \\
\hline \multicolumn{8}{|c|}{ Heterogeneity: $\operatorname{Tau}^{2}=0 ; \mathrm{Chi}^{2}=0.13, \mathrm{df}=1(\mathrm{P}=0.72) ; \mathrm{I}^{2}=0 \%$} \\
\hline \multicolumn{8}{|l|}{ 6.2.2 TAU } \\
\hline Law 2015 & 28 & $44.8(9.5)$ & 23 & $43.7(6.5)$ & $\square$ & $14.9 \%$ & $0.12[-0.43,0.67]$ \\
\hline 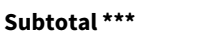 & 28 & & 23 & & & $14.9 \%$ & $0.12[-0.43,0.67]$ \\
\hline \multicolumn{8}{|c|}{ Heterogeneity: Not applicable } \\
\hline \multicolumn{8}{|c|}{ Test for overall effect: $Z=0.43(P=0.67)$} \\
\hline Total ${ }^{\star \star \star}$ & 172 & & 167 & & & $100 \%$ & $0.04[-0.18,0.25]$ \\
\hline \multicolumn{8}{|c|}{ Heterogeneity: $\mathrm{Tau}^{2}=0 ; \mathrm{Chi}^{2}=0.23, \mathrm{df}=2(\mathrm{P}=0.89) ; \mathrm{I}^{2}=0 \%$} \\
\hline \multicolumn{8}{|c|}{ Test for overall effect: $Z=0.34(P=0.73)$} \\
\hline & & & & urs e-health & -0.5 & Favours & arator \\
\hline
\end{tabular}




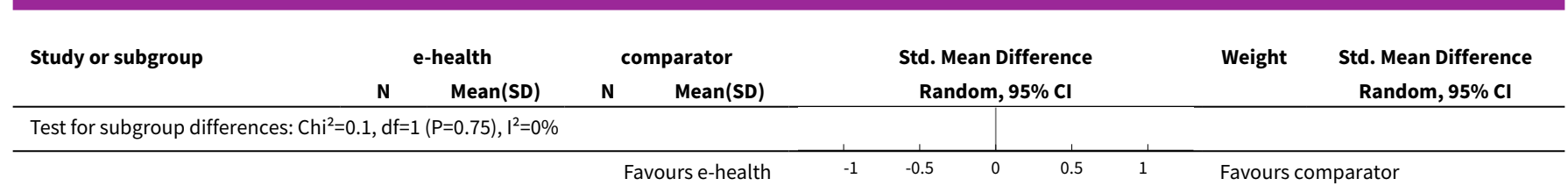

Analysis 6.3. Comparison 6 E-health interventions vs any comparator (by type of comparator), Outcome 3 Anxiety postintervention.

\begin{tabular}{|c|c|c|c|c|c|c|c|}
\hline \multirow[t]{2}{*}{ Study or subgroup } & \multicolumn{2}{|c|}{ e-health } & \multicolumn{2}{|c|}{ comparator } & \multirow{2}{*}{$\begin{array}{c}\text { Std. Mean Difference } \\
\text { Random, } 95 \% \mathrm{Cl}\end{array}$} & \multirow[t]{2}{*}{ Weight } & \multirow{2}{*}{$\begin{array}{c}\text { Std. Mean Difference } \\
\text { Random, } 95 \% \mathrm{Cl}\end{array}$} \\
\hline & $\mathbf{N}$ & Mean(SD) & $\mathbf{N}$ & Mean(SD) & & & \\
\hline \multicolumn{8}{|c|}{ 6.3.1 Attention placebo } \\
\hline Palermo 2016a & 134 & $10.6(5.9)$ & 135 & $10.9(6.1)$ & & $83.21 \%$ & $-0.05[-0.29,0.19]$ \\
\hline 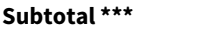 & 134 & & 135 & & & $83.21 \%$ & $-0.05[-0.29,0.19]$ \\
\hline \multicolumn{8}{|c|}{ Heterogeneity: Not applicable } \\
\hline \multicolumn{8}{|c|}{ Test for overall effect: $Z=0.39(P=0.69)$} \\
\hline \multicolumn{8}{|c|}{ 6.3.2 Treatment as usual (TAU) } \\
\hline Law 2015 & 30 & $46.3(9)$ & 25 & $48.3(10.8)$ & $\longrightarrow$ & $16.79 \%$ & $-0.2[-0.73,0.33]$ \\
\hline Subtotal $\star \star \star$ & 30 & & 25 & & & $16.79 \%$ & $-0.2[-0.73,0.33]$ \\
\hline \multicolumn{8}{|c|}{ Heterogeneity: $\mathrm{Tau}^{2}=0 ; \mathrm{Chi}^{2}=0, \mathrm{df}=0(\mathrm{P}<0.0001) ; \mathrm{I}^{2}=100 \%$} \\
\hline \multicolumn{8}{|c|}{ Test for overall effect: $Z=0.73(P=0.46)$} \\
\hline \multicolumn{8}{|c|}{ Heterogeneity: $\mathrm{Tau}^{2}=0 ; \mathrm{Chi}^{2}=0.26, \mathrm{df}=1(\mathrm{P}=0.61) ; \mathrm{I}^{2}=0 \%$} \\
\hline \multicolumn{8}{|c|}{ Test for overall effect: $Z=0.66(P=0.51)$} \\
\hline \multicolumn{8}{|c|}{ Test for subgroup differences: $\mathrm{Chi}^{2}=0.26, \mathrm{df}=1(\mathrm{P}=0.61), \mathrm{I}^{2}=0 \%$} \\
\hline
\end{tabular}

Analysis 6.4. Comparison $6 \mathrm{E}$-health interventions vs any comparator (by type of comparator), Outcome 4 Anxiety 3- to 6-month follow-up.

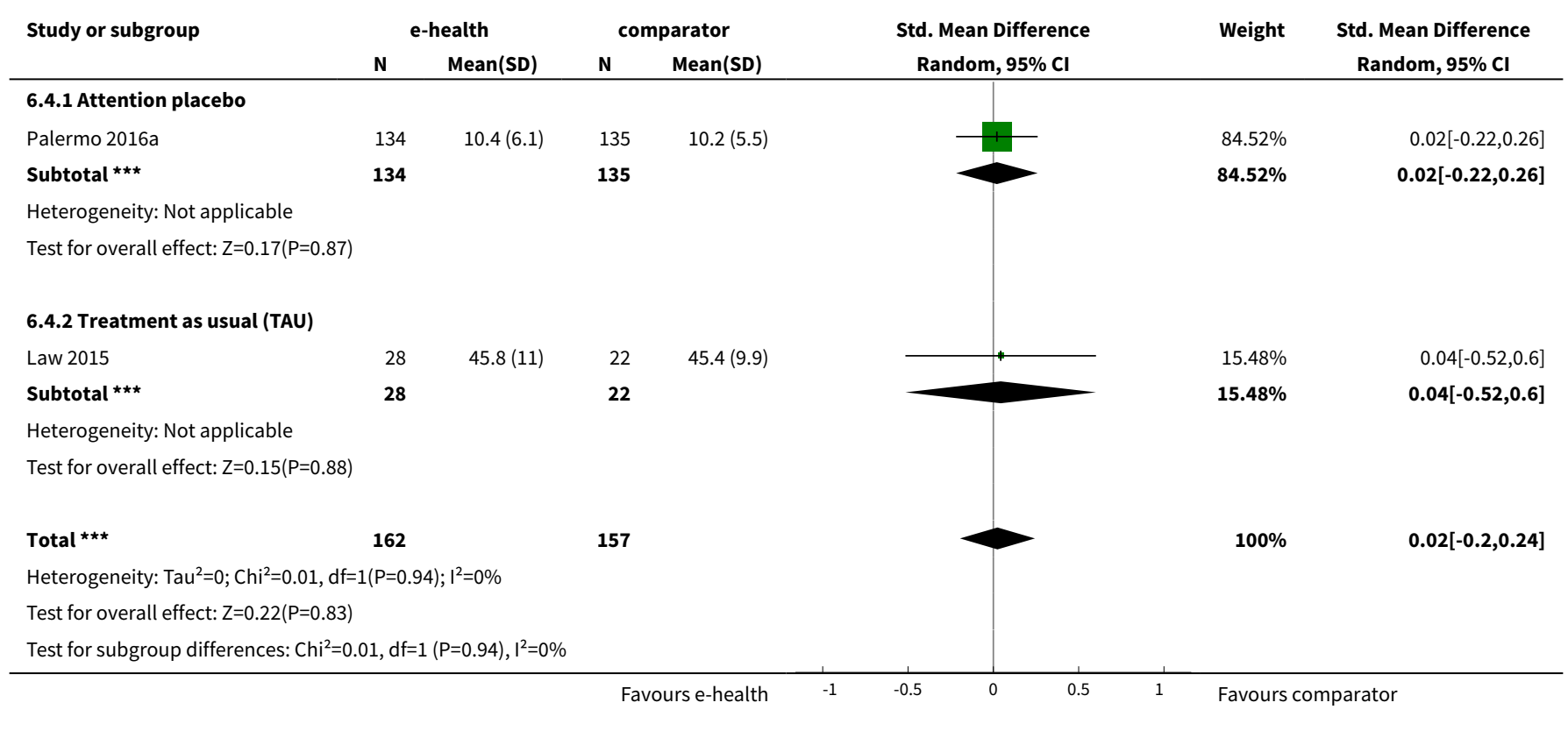


Analysis 6.5. Comparison 6 E-health interventions vs any comparator (by type of comparator), Outcome 5 Treatment acceptability postintervention.

\begin{tabular}{|c|c|c|c|c|c|c|c|}
\hline \multirow[t]{2}{*}{ Study or subgroup } & \multicolumn{2}{|c|}{ e-health } & \multicolumn{2}{|c|}{ comparator } & \multirow{2}{*}{$\begin{array}{c}\text { Std. Mean Difference } \\
\text { Random, } 95 \% \mathrm{Cl}\end{array}$} & \multirow[t]{2}{*}{ Weight } & \multirow{2}{*}{$\begin{array}{c}\text { Std. Mean Difference } \\
\text { Random, } 95 \% \mathrm{Cl}\end{array}$} \\
\hline & $\mathbf{N}$ & Mean(SD) & $\mathbf{N}$ & Mean(SD) & & & \\
\hline \multicolumn{8}{|l|}{ 6.5.1 Attention placebo } \\
\hline Trautmann 2010 & 17 & $2.3(0.6)$ & 18 & $2(0.9)$ & & $100 \%$ & $0.38[-0.29,1.05]$ \\
\hline Subtotal $\star \star \star$ & 17 & & 18 & & & $100 \%$ & $0.38[-0.29,1.05]$ \\
\hline \multicolumn{8}{|l|}{ Heterogeneity: Not applicable } \\
\hline \multicolumn{8}{|l|}{ Test for overall effect: $Z=1.12(P=0.26)$} \\
\hline Total $\star \star \star ~$ & 17 & & 18 & & & $100 \%$ & $0.38[-0.29,1.05]$ \\
\hline \multicolumn{8}{|l|}{ Heterogeneity: Not applicable } \\
\hline \multicolumn{8}{|l|}{ Test for overall effect: $Z=1.12(P=0.26)$} \\
\hline & & & $\mathrm{Fa}$ & urs e-health & -2 & Favours & parator \\
\hline
\end{tabular}

Comparison 7. E-health interventions vs any comparator (by type of long-term physical condition (LTPC))

\begin{tabular}{lllll}
\hline $\begin{array}{l}\text { Outcome or sub- } \\
\text { group title }\end{array}$ & No. of studies & $\begin{array}{l}\text { No. of partici- } \\
\text { pants }\end{array}$ & Statistical method & Effect size \\
\hline $\begin{array}{l}1 \text { Depression postin- } \\
\text { tervention }\end{array}$ & 5 & 441 & Std. Mean Difference (IV, Random, 95\% Cl) & $-0.06[-0.35,0.23]$ \\
\hline 1.1 Pain & 4 & 402 & Std. Mean Difference (IV, Random, 95\% Cl) & $0.05[-0.14,0.25]$ \\
\hline 1.2 Non-pain & 1 & 39 & Std. Mean Difference (IV, Random, 95\% Cl) & $-0.68[-1.33,-0.04]$ \\
\hline
\end{tabular}

Analysis 7.1. Comparison 7 E-health interventions vs any comparator (by type of long-term physical condition (LTPC)), Outcome 1 Depression postintervention.

\begin{tabular}{|c|c|c|c|c|c|c|c|}
\hline \multirow[t]{2}{*}{ Study or subgroup } & \multicolumn{2}{|c|}{ e-health } & \multicolumn{2}{|c|}{ any comparator } & \multirow{2}{*}{$\begin{array}{c}\text { Std. Mean Difference } \\
\text { Random, } 95 \% \mathrm{Cl}\end{array}$} & \multirow[t]{2}{*}{ Weight } & \multirow{2}{*}{$\begin{array}{c}\text { Std. Mean Difference } \\
\text { Random, } 95 \% \mathrm{Cl}\end{array}$} \\
\hline & $\mathbf{N}$ & Mean(SD) & $\mathbf{N}$ & $\operatorname{Mean}(S D)$ & & & \\
\hline \multicolumn{8}{|l|}{ 7.1.1 Pain } \\
\hline Law 2015 & 27 & $46.3(10)$ & 23 & $47.5(9.5)$ & & $17.58 \%$ & $-0.12[-0.68,0.44]$ \\
\hline Palermo 2009 & 26 & $59(13.1)$ & 22 & $61.6(18.7)$ & 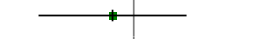 & $17.09 \%$ & $-0.16[-0.73,0.41]$ \\
\hline Palermo 2016a & 134 & $9.7(5.1)$ & 135 & $9.3(5.3)$ & - & $37.47 \%$ & $0.07[-0.16,0.31]$ \\
\hline Trautmann 2010 & 17 & $11(9.2)$ & 18 & $7.7(5.2)$ & & $13.56 \%$ & $0.43[-0.24,1.11]$ \\
\hline Subtotal $\star \star \star$ & 204 & & 198 & & & $85.72 \%$ & $0.05[-0.14,0.25]$ \\
\hline \multicolumn{8}{|c|}{ Heterogeneity: $\operatorname{Tau}^{2}=0 ; \mathrm{Chi}^{2}=2.19, \mathrm{df}=3(\mathrm{P}=0.53) ; \mathrm{I}^{2}=0 \%$} \\
\hline \multicolumn{8}{|c|}{ Test for overall effect: $Z=0.53(P=0.59)$} \\
\hline \multicolumn{8}{|l|}{ 7.1.2 Non-pain } \\
\hline Newcombe 2012 & 19 & $9.5(7.5)$ & 20 & $17.1(13.2)$ & & $14.28 \%$ & $-0.68[-1.33,-0.04]$ \\
\hline Subtotal $\star \star \star$ & 19 & & 20 & & & $14.28 \%$ & $-0.68[-1.33,-0.04]$ \\
\hline \multicolumn{8}{|c|}{ Test for overall effect: $Z=2.07(P=0.04)$} \\
\hline & & & & urs e-health & -0.5 & Favours & mparator \\
\hline
\end{tabular}




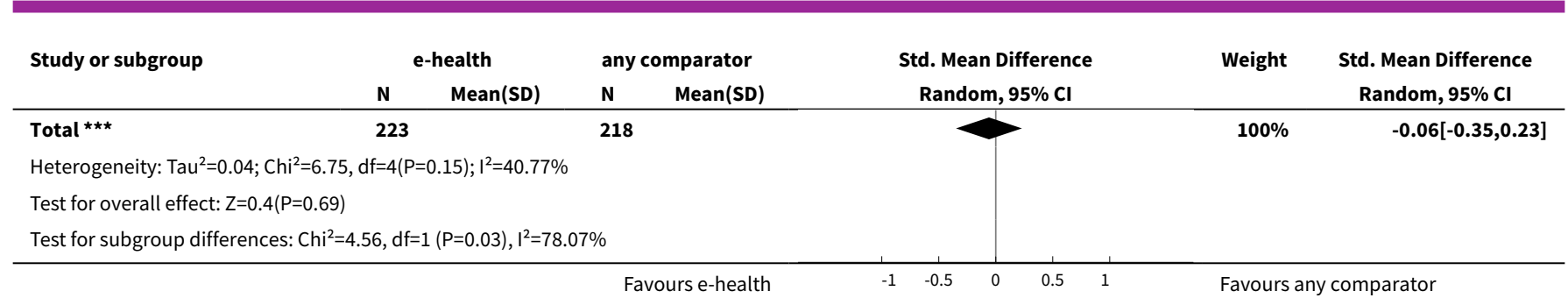

\section{Comparison 8. E-health interventions vs any comparator (by audience: child plus parent vs child only)}

\begin{tabular}{lllll}
\hline $\begin{array}{l}\text { Outcome or subgroup } \\
\text { title }\end{array}$ & No. of studies & $\begin{array}{l}\text { No. of partici- } \\
\text { pants }\end{array}$ & Statistical method & Effect size \\
\hline $\begin{array}{l}1 \text { Depression postinter- } \\
\text { vention }\end{array}$ & 5 & 441 & $\begin{array}{l}\text { Std. Mean Difference (IV, Random, 95\% } \\
\text { Cl) }\end{array}$ & $-0.02[-0.31,0.26]$ \\
\hline 1.1 Child alone & 2 & 74 & $\begin{array}{l}\text { Std. Mean Difference (IV, Random, 95\% } \\
\text { Cl) }\end{array}$ & $-0.13[-1.23,0.97]$ \\
\hline 1.2 Child plus parent & 3 & 367 & $\begin{array}{l}\text { Std. Mean Difference (IV, Random, 95\% } \\
\text { Cl) }\end{array}$ & $0.05[-0.16,0.25]$ \\
\hline
\end{tabular}

Analysis 8.1. Comparison $8 \mathrm{E}-$ health interventions vs any comparator (by audience: child plus parent vs child only), Outcome 1 Depression postintervention.

\begin{tabular}{|c|c|c|c|c|c|c|c|}
\hline \multirow{3}{*}{$\begin{array}{l}\text { Study or subgroup } \\
8.1 .1 \text { Child alone }\end{array}$} & \multicolumn{2}{|c|}{ e-health } & \multicolumn{2}{|c|}{ any comparator } & \multirow{2}{*}{$\begin{array}{c}\text { Std. Mean Difference } \\
\text { Random, } 95 \% \mathrm{Cl}\end{array}$} & \multirow[t]{2}{*}{ Weight } & \multirow{2}{*}{$\begin{array}{c}\text { Std. Mean Difference } \\
\text { Random, } 95 \% \mathrm{Cl}\end{array}$} \\
\hline & \multirow[t]{2}{*}{$\mathbf{N}$} & \multirow[t]{2}{*}{ Mean(SD) } & \multirow[t]{2}{*}{$\mathbf{N}$} & \multirow[t]{2}{*}{$\operatorname{Mean}(S D)$} & & & \\
\hline & & & & & & & \\
\hline Newcombe 2012 & 19 & $9.5(7.5)$ & 20 & $17.1(13.2)$ & $\longrightarrow$ & $14.18 \%$ & $-0.68[-1.33,-0.04]$ \\
\hline Trautmann 2010 & 17 & $11(9.2)$ & 18 & $7.7(5.2)$ & & $13.45 \%$ & $0.43[-0.24,1.11]$ \\
\hline Subtotal $\star \star \star$ & 36 & & 38 & & & $27.63 \%$ & $-0.13[-1.23,0.97]$ \\
\hline \multicolumn{8}{|c|}{ Heterogeneity: $\mathrm{Tau}^{2}=0.51 ; \mathrm{Chi}^{2}=5.52, \mathrm{df}=1(\mathrm{P}=0.02) ; \mathrm{I}^{2}=81.9 \%$} \\
\hline \multicolumn{8}{|c|}{ Test for overall effect: $Z=0.23(P=0.82)$} \\
\hline \multicolumn{8}{|c|}{ 8.1.2 Child plus parent } \\
\hline Law 2015 & 27 & $7.9(6.9)$ & 23 & $7.3(5.7)$ & & $17.51 \%$ & $0.09[-0.47,0.64]$ \\
\hline Palermo 2009 & 26 & $59(13.1)$ & 22 & $61.6(18.7)$ & - & $17 \%$ & $-0.16[-0.73,0.41]$ \\
\hline Palermo 2016a & 134 & $9.7(5.1)$ & 135 & $9.3(5.4)$ & & $37.86 \%$ & $0.07[-0.16,0.31]$ \\
\hline Subtotal *** & 187 & & 180 & & & $72.37 \%$ & $0.05[-0.16,0.25]$ \\
\hline \multicolumn{8}{|c|}{ Test for overall effect: $Z=0.43(P=0.67)$} \\
\hline Total $\star \star \star$ & 223 & & 218 & & & $100 \%$ & $-0.02[-0.31,0.26]$ \\
\hline \multicolumn{8}{|c|}{ Heterogeneity: $\mathrm{Tau}^{2}=0.04 ; \mathrm{Chi}^{2}=6.65, \mathrm{df}=4(\mathrm{P}=0.16) ; \mathrm{I}^{2}=39.86 \%$} \\
\hline \multicolumn{8}{|c|}{ Test for overall effect: $Z=0.16(P=0.88)$} \\
\hline Test for subgroup di & $9, \mathrm{df}=$ & $P=0.76), I^{2}=0 \%$ & & & & & \\
\hline
\end{tabular}




\section{A P P E N D I CES}

\section{Appendix 1. CCMDCT core MEDLINE search}

\section{OVID MEDLINE search strategy, used to inform the Cochrane Common Mental Disorders Group's Specialised Register}

A weekly search alert based on condition + RCT filter only

1. [MeSH Headings]:

eating disorders/ or anorexia nervosa/ or binge-eating disorder/ or bulimia nervosa/ or female athlete triad syndrome/ or pica/ or hyperphagia/ or bulimia/ or self-injurious behavior/ or self mutilation/ or suicide/ or suicidal ideation/ or suicide, attempted/ or mood disorders/ or affective disorders, psychotic/ or bipolar disorder/ or cyclothymic disorder/ or depressive disorder/ or depression, postpartum/ or depressive disorder, major/ or depressive disorder, treatment-resistant/ or dysthymic disorder/ or seasonal affective disorder/ or neurotic disorders/ or depression/ or adjustment disorders/ or exp antidepressive agents/ or anxiety disorders/ or agoraphobia/ or neurocirculatory asthenia/ or obsessive-compulsive disorder/ or obsessive hoarding/ or panic disorder/ or phobic disorders/ or stress disorders, traumatic/ or combat disorders/ or stress disorders, post-traumatic/ or stress disorders, traumatic, acute/ or anxiety/ or anxiety, castration/ or koro/ or anxiety, separation/ or panic/ or exp anti-anxiety agents/or somatoform disorders/or body dysmorphic disorders/ or conversion disorder/ or hypochondriasis/or neurasthenia/ or hysteria/ or munchausen syndrome by proxy/ or munchausen syndrome/ or fatigue syndrome, chronic/ or obsessive behavior/ or compulsive behavior/ or behavior, addictive/ or impulse control disorders/ or firesetting behavior/ or gambling/ or trichotillomania/ or stress, psychological/ or burnout, professional/ or sexual dysfunctions, psychological/ or vaginismus/ or Anhedonia/ or Affective Symptoms/ or *Mental Disorders/

2. [Title/ Author Keywords]:

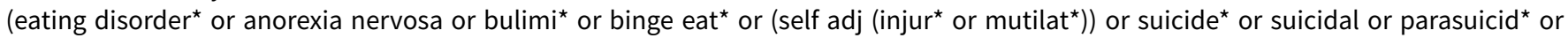
mood disorder ${ }^{\star}$ or affective disorder ${ }^{\star}$ or bipolar i or bipolar ii or (bipolar and (affective or disorder $\left.{ }^{\star}\right)$ ) or mania or manic or cyclothymic ${ }^{\star}$ or depression or depressive or dysthymi* or neurotic or neurosis or adjustment disorder ${ }^{\star}$ or antidepress ${ }^{\star}$ or anxiety disorder ${ }^{\star}$ or agoraphobia or obsess ${ }^{\star}$ or compulsi* or panic or phobi* or ptsd or posttrauma* or post trauma* or combat or somatoform or somati\#ation or medical* unexplained or body dysmorphi* or conversion disorder or hypochondria* or neurastheni* or hysteria or munchausen or chronic fatigue* or gambling or trichotillomania or vaginismus or anhedoni* or affective symptoms or mental disorder ${ }^{\star}$ or mental health).ti,kf.

3. [RCT filter]:

(controlled clinical trial.pt. or randomized controlled trial.pt. or (randomi\#ed or randomi\#ation).ab,ti. or randomly.ab. or (random* adj3 (administ* or allocat ${ }^{\star}$ or assign* or class ${ }^{\star}$ or control ${ }^{\star}$ or determine* or divide* or distribut ${ }^{\star}$ or expose* or fashion or number ${ }^{\star}$ or place or recruit ${ }^{\star}$ or subsitut ${ }^{\star}$ or treat $\left.{ }^{\star}\right)$ ).ab. or placebo*.ab,ti. or drug therapy.fs. or trial.ab,ti. or groups.ab. or (control ${ }^{\star}$ adj3 (trial $^{\star}$ or study or $^{*}$ studies)).ab,ti. or ((singl ${ }^{\star}$ or doubl ${ }^{\star}$ or tripl ${ }^{\star}$ or trebl $\left.l^{\star}\right)$ adj3 (blind ${ }^{\star}$ or mask ${ }^{\star}$ or dummy $\left.{ }^{\star}\right)$ ).mp. or clinical trial, phase ii/ or clinical trial, phase iii/ or clinical trial, phase iv/ or randomized controlled trial/ or pragmatic clinical trial/ or (quasi adj (experimental or random)).ti,ab. or ((waitlist ${ }^{\star}$ or wait* list $^{\star}$ or treatment as usual or TAU) adj3 (control or group)).ab.)

4. (1 and 2 and 3 )

Records are screened for reports of RCTs within the scope of the Cochrane Common Mental Disorders Group. Secondary reports of RCTs are tagged to the appropriate study record. Similar weekly search alerts are also conducted on OVID Embase and PsycINFO, using relevant subject headings (controlled vocabularies) and search syntax, appropriate to each resource.

\section{Appendix 2. Review search: CCMDCTR references register}

The CCMD-CTR-references register was searched using a sensitive set of terms for: age group + condition + comorbidity + eHealth platforms/ computer programs (06/05/16):

\section{[Age Group]}

$\# 1$. (child ${ }^{\star}$ or boy ${ }^{\star}$ or girl ${ }^{\star}$ or infant ${ }^{\star}$ or juvenil ${ }^{\star}$ or minors or paediatric ${ }^{\star}$ or pediatric ${ }^{\star}$ or school ${ }^{\star}$ or preschool ${ }^{*}$ or pre-school ${ }^{*}$ or kindergarten or nursery or adolesc ${ }^{\star}$ or preadolesc ${ }^{\star}$ or pre-adolesc ${ }^{\star}$ or pubert $^{\star}$ or pubescen ${ }^{\star}$ or prepube ${ }^{\star}$ or pre-pube ${ }^{\star}$ or high-school or teen ${ }^{\star}$ or (young next (adult* or people or patient ${ }^{\star}$ or men* or women ${ }^{\star}$ or mother ${ }^{\star}$ or male or female or survivor ${ }^{\star}$ or offender ${ }^{\star}$ or minorit $\left.{ }^{\star}\right)$ ) or youth ${ }^{\star}$ or student* or undergrad* or college or campus or classroom):ti,ab

\section{[Condition: anxiety/depression]}

\#2. ((emotion* or psycholog* or mental) next (health or stress ${ }^{\star}$ or problem ${ }^{\star}$ or disturb* or aspect ${ }^{\star}$ or state ${ }^{\star}$ or ill $)$ ):ti,ab,kw,ky,emt,mh,mc \#3. (depress ${ }^{\star}$ or mood or anxiety or *phobi* or PTSD or post-trauma* or posttrauma or "post trauma*" or panic* or OCD or obsess* or compulsi ${ }^{\star}$ or GAD or "stress disorder*" or "stress reaction"” or "acute stress" or "psychological stress" or "school refusal" or mutism or neurosis or neuroses or neurotic or psychoneuro*):ti,ab,kw,ky,emt, mh, mc

\section{[Comorbidity: chronic physical illness]}

\#4. ("physical* ill*" or "medical* ill*" or "chronic disease" or (chronic* NEXT (ill* or condition" 1 or disease* or disorder* or health)) or (long term NEXT (condition*1 or sick*)) or "medical ${ }^{\star}$ morbid $^{\star}$ " or (medical ${ }^{\star}$ NEXT (comorbid" or co morbid*)) or multimorbid ${ }^{\star}$ or (multi* NEXT (morbid" or "co morbid"” or comorbid* or physical))):ti,ab,kw,ky,emt, mh,mc

\#5. (AIDS or allerg* or angina or aneurysm or "ankylosing spondylitis" or arthropath* or arthriti* or arthrosis or arthroses or asthma* or "atrial fibrillation" or "autoimmune disease*" or "back pain" or blindness or "brain atroph" or (bone NEXT (disease* or disorder*)) or ((bronchi* or bowel) NEXT (disease* or disorder $\left.{ }^{\star}\right)$ ) or bypass or (cancer or neoplasm or neoplastic or malignan ${ }^{\star}$ ) or (cardiac NEXT (arrest or arrhythmia* or surg*)) or cardiomyopath* or ((cardiovascular or coronary) NEAR2 (disease* or disorder* or event $\left.{ }^{\star}\right)$ ) or "cerebral palsy" 
or (cerebrovascular NEAR2 (disease* or disorder* or event*)) or "chronic obstructive" or COPD or pain or cirrhosis or colitis or "congenital abnormalit" or (congential NEAR3 (disease or disorder $\left.{ }^{\star}\right)$ ) or coxarthrosis or Crohn* or Cushing* or "cystic fibrosis" or cystitis) \#6. (deaf* or deformit* or disabled or (physical NEXT (deform* or disab* or impair $\left.{ }^{\star}\right)$ ) or dermatitis or dermato* or dorsopath* or diabet* or "digestive system" ${ }^{\star}$ or duoden* or dystonia or eczema or (endocrine NEXT (disease* or disorder $)$ ) or enuresis or epilep" or "eye disease*" or ("fatigue syndrome" or "chronic fatigue") or fibromyalgia or fibrosis or "food hypersensitivity" or (gastr* NEXT (disease* or disorder*)) or gastritis or "genetic disorder" or gout or (glomerul* NEXT (disease* or disorder $\left.{ }^{\star}\right)$ ) or headache* or ((h?emic or lymph") NEXT (disease* or disorder*)) or h?ematuria or h?emophili* or h?emorrhag* or ((hearing or visual or vision) NEAR2 (aid ${ }^{\star}$ or impair* or loss)) or hemiplegi ${ }^{\star}$ or hepatitis or h? emodialysis or ((renal or kidney) NEXT (disease* or disorder $^{\star}$ or failure)) or (heart NEXT (disease* or disorder* or failure or surg $\left.^{\star}\right)$ ) or HIV or "human immunodeficiency virus" or hypertensi* or hypotensi*)

\#7. ("inflammatory disease*" or incontinen* or "irritable bowel" or isch?emi* or (joint NEXT (disease* or disorder*)) or kyphosis or leuk? emia or ((liver or hepatic) NEXT (disease* or disorder* or failure)) or lordosis or "lung disease*" or "lupus erythemat*" or lymphoma or "macular degeneration" or migraine* or "movement disorder" or musculoskeletal or necrotizing or nephrotic* or neuromuscular or "multiple sclerosis" or myeloma)

\#8. ("nephrotic syndrome" or ((nutritional or metabolic) NEXT (disease* or disorder or syndrome*)) or (organ* NEAR2 (transplant* or recipient $\left.{ }^{\star}\right)$ ) or (neurological NEXT (disease* or disorder*)) or occlusion* or obesity or obese or orthop?edic* or osteo* or "otitis media" or otorhinolaryngology* or otosclerosis or pancrea* or papulosquamous or paraplegi* or parkinson* or "peripheral vascular" or "pick disease*" or pneumoconiosis or polio* or polyarthropath* or polyarteritis or polyarthrosis or polyneuropath* or psoriasis or parapsoriasis or (pulmonary NEAR2 (disease* or disorder $\left.\left.{ }^{\star}\right)\right)$ )

\#9. ((respiratory NEXT (disease* or disorder $\left.{ }^{\star}\right)$ ) or retinopathy or rheumat* or sclerosis or scoliosis or "sickle cell an?emia" or ((skin or "connective tissue") NEXT (disease* or disorder*)) or ("sleep disorder" or "sleep apn?ea" or insomnia* or dyssomnia* or hypersomnia*) or "spina bifida" or "spinal muscular atropy" or spondylo* or stenosis* or stoma* or (stroke or strokes or "cerebral infarct"”) or tetraplegi* or ((thyroid NEAR (disease* or disorder* or dysfunction*)) or hyperthyroidism or hypothyroidism) or tuberculosis or (systemic NEAR (disorder" or disease $\left.{ }^{\star}\right)$ ) or ulcer ${ }^{\star}$ or (urogenital NEXT (disease* or disorder $\left.{ }^{\star}\right)$ ) or vasculopath* or (vascular NEAR (disease* or disorder $\left.{ }^{\star}\right)$ ) or vestibular or ((virus or viral) NEXT disease))

$\# 10$. (\#1 and (\#2 or \#3) and (\#4 or \#5 or \#6 or \#7 or \#8 or \#9))

\section{[eHealth]}

\#11 (android or app or apps or audio* or blog or CBT or CD-ROM or "cell phone" or cellphone or chat or computer* or cyber* or DVD or eHealth or e-health or "electronic health*" or e-Portal or ePortal or eTherap* or e-therap* or forum* or gaming or iCBT or "information technolog*" or "instant messag*" or internet* or ipad or i-pad or iphone or i-phone or ipod or i-pod or web* or WWW or "smart phone" or smartphone or "social network* site*" or "mobile phone" or e-mail* or email* or mHealth or m-health or mobile or multi-media or multimedia or online* or on-line or "personal digital assistant" or PDA or SMS or "social medi*" or software or telecomm ${ }^{\star}$ or telehealth* or telemed* or telemonitor ${ }^{\star}$ or telephone or telepsych* or teletherap* or "text messag*" or texting or podcast or virtual*):ab,ti,kw,ky,emt, mh,mc

\#12 ("Brave for Teen*" or "Brave for Child" " or "Camp Cope-A-Lot" or "Cool Teens" or Interapy or Memo or Minded or Mindcheck* or "Mood Gym" or Moodgym or Moodhelper or "Mood Helper" or Sparx or "The Journey" or "Think Feel Do")

\#13 (Bebo or "Club Penguin" or Facebook or Franktown or Friendster or Habbo or Jabbersmack or hi5 or iTwixie or MySpace or Orkut or "Sweety High" or Kidzworld or Tumblr or Twitter or Sina Weibo or Yoursphere or YouTube)

\#14 (\#11 or \#12 or \#13)

$\# 15$ (\#10 and \#14)

Key to field codes:

ti: title; ab: abstract; kw: CCMD keywords; ky: additional keywords; emt: EMTREE subject headings; mh:MeSH subject headings; mc: MeSH check words

\section{Appendix 3. Review search: CENTRAL search (via CRSO)}

The Cochrane Central Register of Controlled Trials (CENTRAL) was searched (via the Cochrane Register of Studies Online (CRSO)), using a sensitive set of terms for age group, condition, comorbidity and intervention (to Issue 8, 2017):

\section{[Age Group]}

\#1 (child or boy $^{\star}$ or girl ${ }^{\star}$ or infant $^{\star}$ or juvenil ${ }^{\star}$ or minors or paediatric ${ }^{\star}$ or pediatric ${ }^{\star}$ or school ${ }^{\star}$ or preschool ${ }^{\star}$ or pre-school ${ }^{*}$ or kindergarten or nursery or adolesc ${ }^{\star}$ or preadolesc ${ }^{\star}$ or pre-adolesc or pubert $^{\star}$ or pubescen* or prepube ${ }^{\star}$ or pre-pube ${ }^{\star}$ or high-school or teen* or (young next (adult* or people or patient ${ }^{\star}$ or men ${ }^{\star}$ or women ${ }^{\star}$ or mother ${ }^{\star}$ or male or female or survivor ${ }^{\star}$ or offender ${ }^{\star}$ or minorit $\left.{ }^{\star}\right)$ ) or youth ${ }^{\star}$ or student ${ }^{\star}$ or undergrad* or college or campus or classroom):ti,ab

\section{[Condition: anxiety/depression]}

\#2 ((emotion ${ }^{\star}$ or psycholog ${ }^{\star}$ or mental) next (health or stress ${ }^{\star}$ or problem ${ }^{\star}$ or disturb* or aspect $^{\star}$ or state ${ }^{\star}$ or ill*))

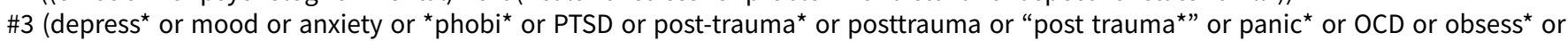

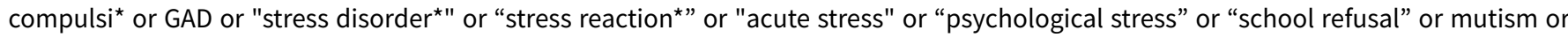
neurosis or neuroses or neurotic or psychoneuro*)

\section{[Comorbidity: chronic physical illness]}

\#4 ("physical* ill*" or "medical" ill*” or "chronic disease" or (chronic* NEXT (ill* or condition* 1 or disease* or disorder" or health)) or (long term NEXT (condition*1 or sick $\left.{ }^{\star}\right)$ ) or “medical ${ }^{\star}$ morbid $^{\star}$ ” or (medical ${ }^{\star}$ NEXT (comorbid* or co morbid*)) or multimorbid* or (multi* NEXT (morbid* or "co morbid" or comorbid* or physical))) 
\#5 (allerg* or angina or aneurysm or "ankylosing spondylitis" or arthropath* or arthriti* or arthrosis or arthroses or asthma* or "atrial fibrillation" or "autoimmune disease" or "back pain" or blindness or "brain atroph" or (bone NEXT (disease* or disorder")) or ((bronchi" or bowel) NEXT (disease* or disorder $\left.{ }^{\star}\right)$ ) or bypass or (cancer or neoplasm* or neoplastic or malignan*) or (cardiac NEXT (arrest or arrhythmia* or surg*)) or cardiomyopath* or ((cardiovascular or coronary) NEAR2 (disease* or disorder* or event $\left.{ }^{\star}\right)$ ) or "cerebral palsy" or (cerebrovascular NEAR2 (disease* or disorder* or event*)) or "chronic obstructive" or COPD or pain or cirrhosis or colitis or "congenital abnormalit" "or (congential NEAR3 (disease or disorder*)) or coxarthrosis or Crohn* or Cushing* or "cystic fibrosis" or cystitis) \#6 (deaf* or deformit* or disabled or (physical NEXT (deform* ${ }^{\star}$ or disab* or impair $\left.{ }^{\star}\right)$ ) or dermatitis or dermato* or dorsopath* or diabet* or "digestive system" " or duoden* or dystonia or eczema or (endocrine NEXT (disease* or disorder )) or enuresis or epilep" or "eye disease*" or ("fatigue syndrome" or "chronic fatigue") or fibromyalgia or fibrosis or "food hypersensitivity" or (gastr* NEXT (disease* or disorder*)) or gastritis or "genetic disorder" or gout or (glomerul* NEXT (disease* or disorder")) or headache* or ((h?emic or lymph") NEXT (disease* or disorder $\left.{ }^{\star}\right)$ ) or h?ematuria or h?emophili* or h?emorrhag ${ }^{\star}$ or ((hearing or visual or vision) NEAR2 (aid* or impair ${ }^{\star}$ or loss)) or hemiplegi ${ }^{\star}$ or hepatitis or h?emodialysis or ((renal or kidney) NEXT (disease* or disorder $^{\star}$ or failure)) or (heart NEXT (disease* or disorder ${ }^{\star}$ or failure or surg $\left.^{\star}\right)$ ) or HIV or "human immunodeficiency virus" or hypertensi* or hypotensi*)

\#7 ("inflammatory disease"” or incontinen* or "irritable bowel" or isch?emi* or (joint NEXT (disease* or disorder*)) or kyphosis or leuk? emia or ((liver or hepatic) NEXT (disease* or disorder* or failure)) or lordosis or "lung disease*" or "lupus erythemat" or lymphoma or "macular degeneration" or migraine* or "movement disorder" or musculoskeletal or necrotizing or nephrotic* or neuromuscular or "multiple sclerosis" or myeloma)

\#8 ("nephrotic syndrome" or ((nutritional or metabolic) NEXT (disease* or disorder or syndrome*)) or (organ* NEAR2 (transplant* or recipient $\left.{ }^{\star}\right)$ ) or (neurological NEXT (disease* or disorder $\left.{ }^{\star}\right)$ ) or occlusion* or obesity or obese or orthop?edic ${ }^{\star}$ or osteo* or "otitis media" or otorhinolaryngology* or otosclerosis or pancrea* or papulosquamous or paraplegi* or parkinson* or "peripheral vascular" or "pick disease " or pneumoconiosis or polio* or polyarthropath* or polyarteritis or polyarthrosis or polyneuropath* or psoriasis or parapsoriasis or (pulmonary NEAR2 (disease* or disorder $\left.\left.{ }^{\star}\right)\right)$ )

\#9 ((respiratory NEXT (disease* or disorder $\left.{ }^{\star}\right)$ ) or retinopathy or rheumat* or sclerosis or scoliosis or "sickle cell an?emia" or ((skin or "connective tissue") NEXT (disease* or disorder")) or ("sleep disorder" or "sleep apn?ea" or insomnia* or dyssomnia* or hypersomnia*) or "spina bifida" or "spinal muscular atropy" or spondylo* or stenosis" or stoma* or (stroke or strokes or "cerebral infarct") or tetraplegi* or ((thyroid NEAR (disease* or disorder* or dysfunction*)) or hyperthyroidism or hypothyroidism) or tuberculosis or (systemic NEAR (disorder" or disease $\left.{ }^{\star}\right)$ ) or ulcer ${ }^{\star}$ or (urogenital NEXT (disease ${ }^{\star}$ or disorder $\left.{ }^{\star}\right)$ ) or vasculopath ${ }^{\star}$ or (vascular NEAR (disease* or disorder $\left.^{\star}\right)$ ) or vestibular or ((virus or viral) NEXT disease))

$\# 10$ ((\#1 and (\#2 or \#3) and (\#4 or \#5 or \#6 or \#7 or \#8 or \#9))

[Intervention: psychological therapies]

\#11 MESH DESCRIPTOR Psychotherapy EXPLODE ALL TREES

\#12 ((psychologic* or behavio?r or cognitive) adj3 (intervent* or therap $^{\star}$ or treat* or manag $\left.\left.^{\star}\right)\right)$ :ti,ab

\#13 (abreaction or "acting out" or (acceptance NEAR2 commitment) or "activity scheduling" or adlerian or "analytical therap*" or "anger control" or "anger management" or "art therap" or "assertive* training" or "attention bias modification" or "autogenic training" or autosuggestion or "aversion therap" or "balint group" or "behavio* activation" or "behavio* contracting" or "behavio* modification" or "behavio* therap" "or bibliotherap* or "body therap*" or "brief therapy" or catharsis or "client cent" therapy" or "cognitive behavio*"or "cognitive therap" or CBT or CCBT or iCBT or "cognitive rehabilitation" or "cognitive restructur*" or "colour therap" or "color therap" or "compassion focus" or "compassionate therap*" or "conjoint therap" "or "contingency management" or "conversion therap" or "conversational therap" or countertransference or "coping skill*" or counsel" or "covert sensitization" or "crisis intervention" or "crisis management")

\#14 ((dialectic* NEAR2 therap*) or "diffusion therap*" or "distraction therap*" or (dream* NEAR3 analys") or "eclectic therap*" or "emotion* focus ${ }^{\star}$ therap ${ }^{\star}$ or "emotional freedom technique" or "encounter group therap" or "expressive therap" or "eye movement desensiti\#ation" or "family therap" or "focus oriented" or "free association" or freudian or "functional analysis" or gestalt or griefwork or "group therap*" or "guided image*" or "holistic therap*" or humanistic or hypnosis or hypnotherapy or hypnoti\#zability or "implosive therap*" or "insight therap*" or "integrative therap*" or "interpersonal therap*" or Jungian or kleinian)

\#15 (logotherap* or "logo therap*" or meditation or "mental healing" or metacognitive or meta-cognitive or milieu or "mind train*" or mindfulness or morita or "multimodal therap" or music or "narrative therap*" or "nondirective therap ${ }^{\star}$ " or non-directive therap ${ }^{\star}$ or "nondirective therap*" or "non-specific therap*" or "nonspecific therap*" or "object relations" or "personal construct therap*" or "person cent $^{\star}$ therap ${ }^{\star \prime}$ or "persuasion therap ${ }^{\star "}$ or "pet therap" or "animal therap*" or "play therap*" or ((pleasant or pleasing) NEAR2 event ${ }^{\star}$ ) or "present cent* therap*" or "primal therap*" or "problem focus* therap*" or "problem sol*" or "process experiential" or psychoanaly* or psychodrama or psychodynamic or psychoeducat* or psychotherap*)

\#16 ("rational emotive" or "reality therap*" or "reciprocal inhibition" or "relationship therap*" or "relaxation stress management" or "relaxation technique*" or "relaxation therap*" or "relaxation training" or "reminiscence therap*" or rogerian or "role play*" or schema or "self analys" or "self esteem building" or "sensitivity training" or "sleep phase chronotherap ${ }^{\star}$ " or "socioenvironment* therap ${ }^{\star "}$ or "social skill*" or sociotherap* or "solution focused therap" or "stress management" or "support group*" or (support NEAR3 psycho*) or "supportive therap " or "systematic desensiti*" or "systemic *therap" or "therapeutic communit" or "therapeutic technique" or "third wave" or "time limited therap*" or "transference therap*" or "transactional analysis" or transtheoretical or "validation therap*")

\section{[Intervention: eHealth]}

\#17 (Bebo or "Club Penguin" or Facebook or Franktown or Friendster or Habbo or Jabbersmack or hi5 or iTwixie or MySpace or Orkut or "Sweety High" or Kidzworld or Tumblr or Twitter or Sina Weibo or Yoursphere or YouTube) 
\#18 ("Brave for Teen"” or "Brave for Child"” or "Camp Cope-A-Lot" or "Cool Teens" or Interapy or Memo or Minded or Mindcheck* or "Mood Gym" or Moodgym or Moodhelper or "Mood Helper" or Sparx or "The Journey" or "Think Feel Do")

\#19 (android or app or apps or blog or "cell phone" or cellphone or "chat room" or computer* or cyber* or DVD or eHealth or e-health or "electronic health*" or e-Portal or ePortal or eTherap* or e-therap* or forum* or gaming or CCBT or iCBT or "information technolog*" or "instant messag*" or internet* or ipad or i-pad or iphone or i-phone or ipod or i-pod or web* or WWW or "smart phone" or smartphone or "social network" site" or "mobile phone" or e-mail* or email* or mHealth or m-health or mobile or multi-media or multimedia or online* or on-line or "personal digital assistant" or PDA or SMS or "social medi*" or software or telecomm* or telehealth* or telemed* or telemonitor or telepsych*or teletherap* or "text messag*" or texting or podcast or virtual ${ }^{\star}$ )

\#20 (\#11 OR \#12 OR \#13 OR \#14 OR \#15 OR \#16 OR \#17 OR \#18 OR \#19)

\#21 (\#10 AND \#20)

\section{Appendix 4. Review search update 2017}

In compliance with Cochrane MECIR standard C37 (searches to be rerun within 12 months of publication), CCMD's information specialist ran an update search on 18 August 2017, details below.

CENTRAL retrieved 209 records and Ovid XSearch 941. These were de-duplicated against each other and records retrieved in 2016, leaving 900 new records to screen.

\section{CENTRAL}

CENTRAL was searched (via the Cochrane Register of Studies Online (CRSO)) from Issue 6, 2016 to Issue 8, 2017. The search terms are listed in Appendix 3.

\section{Ovid XSearch (MEDLINE, Embase, PsycINFO)}

In August 2017, CCMD's Information Specialist also ran a cross-search of Ovid databases (as the Group's Specialised Register (CCMD-CTR) was out of date at this time).

Ovid databases searched: MEDLINE(R) Epub Ahead of Print, In-Process \& Other Non-Indexed Citations, MEDLINE(R) Daily and MEDLINE(R) 1946 to 18-Aug-2017, Embase 1974 to 2017 Week 33, PsycINFO 1806 to August Week 22017.

Date limited: 1 Jan 2016 to 18-Aug-2017.

\section{Search Terms:}

1. (child ${ }^{\star}$ or boy ${ }^{\star}$ or girl ${ }^{\star}$ or infant ${ }^{\star}$ or juvenil ${ }^{\star}$ or minors or paediatric ${ }^{\star}$ or pediatric ${ }^{\star}$ or school ${ }^{\star}$ or preschool ${ }^{\star}$ or pre-school ${ }^{\star}$ or kindergarten or nursery or adolesc ${ }^{\star}$ or preadolesc ${ }^{\star}$ or pre-adolesc ${ }^{\star}$ or pubert $^{\star}$ or pubescen ${ }^{\star}$ or prepube or pre-pube $^{\star}$ or high-school or teen ${ }^{\star}$ or (young adj3 (adult ${ }^{\star}$ or people or patient ${ }^{\star}$ or men ${ }^{\star}$ or women ${ }^{\star}$ or mother ${ }^{\star}$ or male or female or survivor ${ }^{\star}$ or offender ${ }^{\star}$ or minorit ${ }^{\star}$ )) or youth ${ }^{\star}$ or student $^{\star}$ or undergrad ${ }^{\star}$ or college or campus or classroom).ti,ab,kf,kw,id,hw.

2. ((emotion ${ }^{\star}$ or psycholog* or mental) adj3 (health or stress ${ }^{\star}$ or problem ${ }^{\star}$ or disturb* or aspect ${ }^{\star}$ or state* or ill $\left.{ }^{\star}\right)$ ).ti,ab,kf,kw,id,hw.

3. (depress ${ }^{\star}$ or mood or anxiety or agoraphobi* or phobi* or PTSD or post-trauma* or posttrauma or post trauma* or panic* or OCD or obsess $^{\star}$ or compulsi ${ }^{\star}$ or GAD or stress disorder* or stress reaction* or acute stress or psychological stress or school refusal or mutism or neurosis or neuroses or neurotic or psychoneuro*).ti,ab,kf,kw,id,hw.

4. or/2-3

5. (physical ${ }^{\star}$ ill $^{\star}$ or medical ${ }^{\star}$ ill $^{\star}$ or chronic disease or (chronic ${ }^{\star}$ adj3 (ill ${ }^{\star}$ or condition ${ }^{\star} 1$ or disease or disorder $^{\star}$ or health)) or (long term

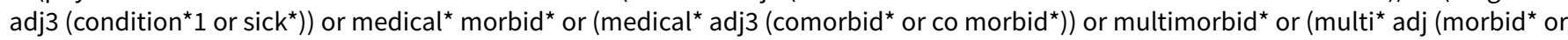
co morbid* or comorbid* or physical))).ti,ab,kf,kw,id,hw.

6. (allerg* or angina or aneurysm or ankylosing spondylitis or arthropath* or arthriti* or arthrosis or arthroses or asthma* or atrial fibrillation or autoimmune disease ${ }^{\star}$ or back pain or blindness or brain atroph* or (bone adj (disease* or disorder $\left.{ }^{\star}\right)$ ) or ((bronchi ${ }^{\star}$ or bowel) adj (disease* or disorder $\left.{ }^{\star}\right)$ ) or bypass or (cancer or neoplasm* or neoplastic or malignan*) or (cardiac adj (arrest or arrhythmia* or surg*)) or cardiomyopath* or ((cardiovascular or coronary) adj2 (disease* or disorder* or event $\left.{ }^{\star}\right)$ ) or cerebral palsy or (cerebrovascular adj2 (disease* or disorder* or event $\left.{ }^{\star}\right)$ ) or chronic obstructive or COPD or pain or cirrhosis or colitis or congenital abnormalit* or (congential adj3 (disease or disorder $\left.{ }^{\star}\right)$ ) or coxarthrosis or Crohn* or Cushing* or cystic fibrosis or cystitis).ti,ab,kf,kw,id,hw.

7. (deaf* or deformit* or disabled or (physical adj (deform* or disab* or impair $\left.{ }^{\star}\right)$ ) or dermatitis or dermato* or dorsopath* or diabet* or digestive system ${ }^{\star}$ or duoden ${ }^{\star}$ or dystonia or eczema or (endocrine adj (disease* or disorder $\left.{ }^{\star}\right)$ ) or enuresis or epilep* or eye disease ${ }^{\star}$ or (fatigue syndrome or chronic fatigue) or fibromyalgia or fibrosis or food hypersensitivity or (gastr ${ }^{\star}$ adj (disease* or disorder $\left.{ }^{\star}\right)$ ) or gastritis or genetic disorder ${ }^{\star}$ or gout or (glomerul* adj (disease* or disorder $\left.{ }^{\star}\right)$ ) or headache* or ((h?emic or lymph $\left.{ }^{\star}\right)$ adj (disease* or disorder $\left.{ }^{\star}\right)$ ) or h? ematuria or h?emophili* or h?emorrhag* or ((hearing or visual or vision) adj2 (aid* or impair ${ }^{\star}$ or loss)) or hemiplegi* or hepatitis or h? emodialysis or ((renal or kidney) adj (disease or disorder $^{\star}$ or failure)) or (heart adj (disease* or disorder ${ }^{\star}$ or failure or surg $\left.{ }^{\star}\right)$ ) or HIV or human immunodeficiency virus or hypertensi ${ }^{\star}$ or hypotensi $\left.{ }^{\star}\right) . t i, a b, k f, k w, i d, h w$.

8. (inflammatory disease* or incontinen* or irritable bowel or isch?emi ${ }^{\star}$ or (joint adj (disease* or disorder $\left.{ }^{\star}\right)$ ) or kyphosis or leuk? emia or ((liver or hepatic) adj (disease ${ }^{\star}$ or disorder ${ }^{\star}$ or failure)) or lordosis or lung disease* or lupus or lymphoma or macular degeneration or migraine $e^{\star}$ or movement disorder* or musculoskeletal or necrotizing or nephrotic* or neuromuscular or multiple sclerosis or myeloma).ti,ab,kf,kw,id,hw.

9. (nephrotic syndrome or ((nutritional or metabolic) adj (disease* or disorder or syndrome*)) or ((organ* or kidney or stem cell) adj2 (transplant ${ }^{\star}$ or recipient $\left.{ }^{\star}\right)$ ) or (neurological adj (disease* or disorder $\left.{ }^{\star}\right)$ ) or occlusion ${ }^{\star}$ or obesity or obese or orthop? edic ${ }^{\star}$ or osteo* or otitis

E-Health interventions for anxiety and depression in children and adolescents with long-term physical conditions (Review) 
media or otorhinolaryngolog* or otosclerosis or pancrea* or papulosquamous or paraplegi* or parkinson* or (peripheral adj (arterial or vascular)) or pick disease ${ }^{\star}$ or pneumoconiosis or polio* or polyarthropath* or polyarteritis or polyarthrosis or polyneuropath ${ }^{\star}$ or psoriasis or parapsoriasis or (pulmonary adj2 (disease* or disorder*))).ti,ab,kf,kw,id,hw.

10. ((respiratory adj (disease* or disorder $)$ ) or retinopathy or rheumat* or sclerosis or scoliosis or sickle cell an?emia or ((skin or connective tissue) adj (disease ${ }^{\star}$ or disorder $\left.{ }^{\star}\right)$ ) or (sleep disorder* or sleep apn?ea or insomnia* or dyssomnia* or hypersomnia*) or spina bifida or spinal muscular atropy or spondylo* or stenosis* or stoma* or (stroke or strokes or cerebral infarct*) or tetraplegi* or ((thyroid adj (disease* or disorder ${ }^{\star}$ or dysfunction*)) or hyperthyroidism or hypothyroidism) or tuberculosis or (systemic adj5 (disorder ${ }^{\star}$ or disease $\left.^{\star}\right)$ ) or ulcer ${ }^{\star}$ or (urogenital adj (disease* or disorder $\left.^{\star}\right)$ ) or vasculopath ${ }^{\star}$ or (vascular adj5 (disease* or disorder $\left.{ }^{\star}\right)$ ) or vestibular or ((virus or viral) adj disease)).ti,ab,kf,kw,id,hw.

11. or/5-10

12. exp Psychotherapy/ or exp Psychotherapeutic Techniques/

13. exp Child Psychotherapy/ or exp Adolescent Psychotherapy/

14. ((psychologic ${ }^{\star}$ or behavio?r or cognitive) adj3 (intervent ${ }^{\star}$ or therap* or treat* or manag*)).ti,ab,id,kf,kw.

15. (abreaction or acting out or (acceptance adj2 commitment) or activity scheduling or adlerian or analytical therap* or anger control or anger management or art therap* or assertive* training or attention bias modification or autogenic training or autosuggestion or aversion therap* or balint or behavio* activation or behavio* contracting or behavio* modification or behavio* therap* or bibliotherap* or body therap* or brief therapy or catharsis or client cent ${ }^{\star}$ therapy or cognitive behavio* or cognitive therap* or CBT or CCBT or iCBT or cognitive rehabilitation or cognitive restructur ${ }^{\star}$ or colour therap ${ }^{\star}$ or color therap ${ }^{\star}$ or compassion focus* or compassionate therap* ${ }^{\star}$ or conjoint therap ${ }^{\star}$ or contingency management or conversion therap ${ }^{\star}$ or conversational therap* or countertransference or coping skill* or counsel ${ }^{\star}$ or covert sensitization or crisis intervention or crisis management).ti,ab,kf,kw,id,hw.

16. ((dialectic ${ }^{\star}$ adj2 therap*) or diffusion therap* or distraction therap ${ }^{\star}$ or (dream* adj3 analys ${ }^{\star}$ ) or eclectic therap ${ }^{\star}$ or emotion $^{\star}$ focus ${ }^{\star}$ therap ${ }^{\star}$ or emotional freedom technique or encounter group therap ${ }^{\star}$ or existential or experiential or exposure therap ${ }^{\star}$ or expressive therap ${ }^{\star}$ or eye movement desensiti\#ation or family therap* or focus oriented or free association or freudian or functional analysis or gestalt or griefwork or group therap* or guided image* or holistic therap* or humanistic or hypnosis or hypnotherapy or hypnoti\#zability or implosive therap* or insight therap* or integrative therap* or interpersonal therap* or Jungian or kleinian).ti,ab,id,kf,kw,hw.

17. (logotherap* or logo therap* or meditation or mental healing or metacognitive or meta-cognitive or milieu or mind train ${ }^{\star}$ or mindfulness or morita or multimodal therap* or music or narrative therap* or nondirective therap* or non-directive therap* or nondirective therap ${ }^{\star}$ or non-specific therap ${ }^{\star}$ or nonspecific therap ${ }^{\star}$ or object relations or personal construct therap ${ }^{\star}$ or person cent ${ }^{\star}$ therap ${ }^{\star}$ or persuasion therap ${ }^{\star}$ or pet therap* or animal therap ${ }^{\star}$ or play therap* or ((pleasant or pleasing) adj2 event ${ }^{\star}$ ) or present cent ${ }^{\star}$ therap ${ }^{\star}$ or primal therap $^{\star}$ or problem focus ${ }^{\star}$ therap* or problem sol* or process experiential or psychoanaly* or psychodrama or psychodynamic or psychoeducat ${ }^{\star}$ or psychotherap*).ti,ab,kf,kw,id,hw.

18. (rational emotive or reality therap* or reciprocal inhibition or relationship therap* or relaxation stress management or relaxation technique* or relaxation therap* or relaxation training or reminiscence therap* or rogerian or role play ${ }^{\star}$ or schema or self analys ${ }^{\star}$ or self esteem building or sensitivity training or sleep phase chronotherap* or socioenvironment ${ }^{\star}$ therap* ${ }^{\star}$ or social skill* or sociotherap* or solution focused therap* or stress management or support group ${ }^{\star}$ or (support adj3 psycho*) or supportive therap ${ }^{\star}$ or systematic desensiti ${ }^{\star}$ or systemic *therap* or therapeutic communit* or therapeutic technique or third wave or time limited therap* ${ }^{\star}$ or transference therap* ${ }^{\star}$ or transactional analysis or transtheoretical or validation therap*).ti,ab,kf,kw,id,hw.

19. (Bebo or Club Penguin or Facebook or Franktown or Friendster or Habbo or Jabbersmack or hi5 or iTwixie or MySpace or Orkut or Sweety High or Kidzworld or Tumblr or Twitter or Sina Weibo or Yoursphere or YouTube).ti,ab,kf,kw,id,hw.

20. (Brave for Teen ${ }^{\star}$ or Brave for Child* or Camp Cope-A-Lot or Cool Teens or Interapy or Memo or Minded or Mindcheck* or Mood Gym or Moodgym or Moodhelper or Mood Helper or Sparx or The Journey or Think Feel Do).ti,ab,kf,kw,id,hw.

21. (android or app or apps or blog or cell phone or cellphone or chat room or computer ${ }^{\star}$ or cyber* or DVD or eHealth or e-health or electronic health* or e-Portal or ePortal or eTherap* or e-therap* or forum* or gaming or CCBT or iCBT or information technolog* or instant messag* $^{\star}$ or internet ${ }^{\star}$ or ipad or i-pad or iphone or i-phone or ipod or i-pod or web* or WWW or smart phone or smartphone or social

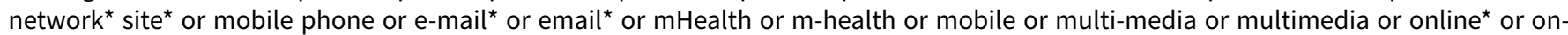
line or personal digital assistant or PDA or SMS or social medi ${ }^{\star}$ or software or telecomm ${ }^{\star}$ or telehealth* or telemed $^{\star}$ or telemonitor $^{\star}$ or telepsych*or teletherap* ${ }^{\star}$ or text messag* or texting or podcast or virtual $\left.{ }^{\star}\right): t i, k f, k w, i d, h w$.

22. or $/ 12-21$

23. trial.ti.

24. (randomi\#ed or randomi\#ation or randomi\#ing).ti,ab,kf,kw,id.

25. (RCT or at random or (random* adj3 (assign* or allocat* or control* or crossover or cross-over or design ${ }^{\star}$ or divide* or division or number))).ti,ab,kf,kw,id.

26. placebo.hw,ti,ab,kf,kw,id.

27. ((control ${ }^{\star}$ adj2 (trial or study or group)) and (placebo or waitlist ${ }^{\star}$ or wait $^{\star}$ list $^{\star}$ or ((treatment or care) adj2 usual))).ti,ab,kf,kw,id,hw.

28. Randomized Controlled Trial.sh,pt.

29. Double Blind Procedure/

30. Double Blind Method/

31. (clinical trial or empirical study).md.

32. ((single or double or triple) adj2 (blind* or mask* or dummy)).ti,ab,kf,kw,id.

33. or/23-32

34. ((animal or nonhuman) not (human and (animal or nonhuman))).hw.

35. (33 not 34)

E-Health interventions for anxiety and depression in children and adolescents with long-term physical conditions (Review)

Copyright (c) 2018 The Cochrane Collaboration. Published by John Wiley \& Sons, Ltd. 
36. ( 1 and 4 and 11 and 22 and 35 )

37. $\left(2016^{\star}\right.$ or $\left.2017^{\star}\right) \cdot y r, e m, d d, d c, e d$.

38. (36 and 37)

39. (case adj (control* or report?)).ti,kf,kw,id,hw.

40. (review or letter or comment $\left.{ }^{\star}\right)$.ti,hw,pt.

41. (dental or dentist ${ }^{\star}$ or an?esthes*).ti,hw,jw.

42. or/39-41

43. (38 not 42$)$

WHAT'S NEW

\begin{tabular}{lll}
\hline Date & Event & Description \\
\hline 31 August 2018 & Amended & Correction of link to the Characteristics of included studies table \\
\hline
\end{tabular}

CONTRIBUTIONS OF AUTHORS

\begin{tabular}{|c|c|}
\hline Task & Who undertook the task? \\
\hline Draft the protocol & Hiran Thabrew \\
\hline $\begin{array}{l}\text { Develop a search strategy (in conjunction with CCMDs Information Spe- } \\
\text { cialist) }\end{array}$ & Hiran Thabrew, Karolina Stasiak, Stephen Wong \\
\hline $\begin{array}{l}\text { Select which trials to include ( } 2 \text { people }+1 \text { arbiter in the event of dis- } \\
\text { pute) }\end{array}$ & Hiran Thabrew, Karolina Stasiak and Stephen Wong \\
\hline Extract data from trials ( 2 people +1 arbiter in the event of dispute) & Hiran Thabrew, Jessica Huss and Karolina Stasiak \\
\hline $\begin{array}{l}\text { Undertake 'Risk of bias' assessments ( } 2 \text { people }+1 \text { arbiter in the event } \\
\text { of dispute) }\end{array}$ & Hiran Thabrew, Sarah Hetrick, Karolina Stasiak \\
\hline Enter data into RevMan 5 (Cochrane software) & Hiran Thabrew, Karolina Stasiak \\
\hline Carry out the analysis & Hiran Thabrew, Sarah Hetrick \\
\hline Interpret the analysis & Hiran Thabrew, Sarah Hetrick, Sally Merry \\
\hline Draft the final review & $\begin{array}{l}\text { Hiran Thabrew, Karolina Stasiak, Sarah Hetrick, Sally Mer- } \\
\text { ry }\end{array}$ \\
\hline Produce the 'Summary of findings' tables & Hiran Thabrew \\
\hline $\begin{array}{l}\text { Check final review meets all mandatory MECIR standards before sub- } \\
\text { mission }\end{array}$ & Hiran Thabrew \\
\hline Keep the review up to date & $\begin{array}{l}\text { Hiran Thabrew, Karolina Stasiak, Sarah Hetrick, Sally Mer- } \\
\text { ry }\end{array}$ \\
\hline
\end{tabular}

\section{DECLARATIONS OF INTEREST}

Sally Merry and Karolina Stasiak have been involved in designing and trialing SPARX, an online and CD-ROM based interactive health game for adolescents with depression. 


\section{SOURCES OF SUPPORT}

\section{Internal sources}

- University of Auckland, New Zealand.

Salaries of authors

\section{External sources}

- Oakley Foundation, New Zealand.

Equipment and research assistance

- Starship Foundation, New Zealand.

Equipment and research assistance

- National Institute for Health Research (NIHR), UK.

Single largest funder of the CCMD group

\section{DIFFERENCES BETWEEN PROTOCOL AND REVIEW}

We made five amendments to the plan outlined in our review protocol. Firstly, we did not conduct a cited reference search on the Web of Science database as planned. Secondly, due to the small number of identified trials, we included an additional comparison group, ehealth interventions versus any comparator and based our Summary of Findings table on this group. Thirdly, as a number of trials had undertaken quantitative analyses of treatment acceptability, we decided to triangulate our judgement regarding treatment acceptability by including: i) quantitative measures of acceptability, ii) the number of dropouts, and iii) adverse events. Fourthly, as Covidence ${ }^{\circledR}$ software became available following the drafting of the review protocol, we extracted data using this software, and not the data extraction sheet that was described in the protocol. Finally, we ensured that it was clear in our methods that where only one trial was included in the metaanalysis that the mean difference was used (and included in the text an explanation that this would also be used in the case where an outcome was measured by the same scale across trials, which did not occur in this review) and ensured that we were expicit in describing our data synthesis method for meta-analysis (random effects model across all meta-analyses even when only one trial was included for consistency).

\section{INDEX TERMS}

\section{Medical Subject Headings (MeSH)}

Anxiety [ ${ }^{*}$ therapy]; Breathing Exercises [ ${ }^{*}$ methods]; Chronic Disease [ ${ }^{*}$ psychology]; Cognitive Behavioral Therapy [ ${ }^{*}$ methods]; Depression [ ${ }^{\star}$ therapy]; Randomized Controlled Trials as Topic; Telemedicine [ ${ }^{*}$ methods]; Treatment Outcome

\section{MeSH check words}

Adolescent; Child; Humans 